\title{
Anterior cutaneous nerve entrapment syndrome : epidemiology and surgical management
}

Citation for published version (APA):

van Assen, T. (2015). Anterior cutaneous nerve entrapment syndrome : epidemiology and surgical management. [Doctoral Thesis, Maastricht University]. Maastricht University. https://doi.org/10.26481/dis.20150611ta

Document status and date:

Published: 01/01/2015

DOI:

10.26481/dis.20150611ta

Document Version:

Publisher's PDF, also known as Version of record

\section{Please check the document version of this publication:}

- A submitted manuscript is the version of the article upon submission and before peer-review. There can be important differences between the submitted version and the official published version of record. People interested in the research are advised to contact the author for the final version of the publication, or visit the DOI to the publisher's website.

- The final author version and the galley proof are versions of the publication after peer review.

- The final published version features the final layout of the paper including the volume, issue and page numbers.

Link to publication

\footnotetext{
General rights rights.

- You may freely distribute the URL identifying the publication in the public portal. please follow below link for the End User Agreement:

www.umlib.nl/taverne-license

Take down policy

If you believe that this document breaches copyright please contact us at:

repository@maastrichtuniversity.nl

providing details and we will investigate your claim.
}

Copyright and moral rights for the publications made accessible in the public portal are retained by the authors and/or other copyright owners and it is a condition of accessing publications that users recognise and abide by the legal requirements associated with these

- Users may download and print one copy of any publication from the public portal for the purpose of private study or research.

- You may not further distribute the material or use it for any profit-making activity or commercial gain

If the publication is distributed under the terms of Article $25 \mathrm{fa}$ of the Dutch Copyright Act, indicated by the "Taverne" license above, 
Anterior Cutaneous

Nerve Entrapment Syndrome

Epidemiology and surgical

management 


\section{Anterior Cutaneous Nerve Entrapment Syndrome \\ Epidemiology and surgical management}

Een wetenschappelijke proeve op het gebied van de Medische Wetenschappen

\section{Colofon}

Author:

Cover design en lay-out:

Original cover photo:

Printing:

ISBN:
T. van Assen

Miranda Dood, Mirakels Ontwerp

Arno Lataster, Dept. of Anatomy and Embryology Maastricht University

Gildeprint - The Netherlands

978-90-9029031-7

\section{Maastricht University}

Printing of this thesis was financially supported by institutions of the Máxima Medical Center; department of surgery - the MMC Academy - SolviMáx, Center of Excellence for Abdominal Wall and Groin Pain - SMC SportMáx Zuidoost Brabant, and Rabobank Eindhoven-Veldhoven, Medicidesk.

medisch centrum

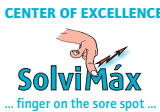

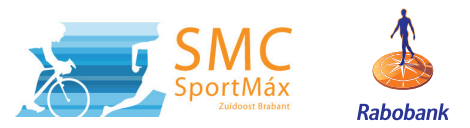

(c) T. van Assen, Eindhoven, The Netherlands, 2015.

All rights reserved. No part of this publication may be reproduced or transmitted

in any form by any means, without permission of the author.

\section{Proefschrift}

ter verkrijging van de graad van doctor aan de Universiteit Maastricht

op gezag van Rector Magnificus prof. dr. L.L.G. Soete, volgens besluit van het College van Decanen,

in het openbaar te verdedigen

op donderdag 11 juni 2015 om 10.00 uur

door

\section{Tijmen van Assen}

Geboren op 3 maart 1986

te Epe 


\section{Promotores}

Prof. dr. G.L. Beets, Maastricht UMC+

Prof. dr. M. van Kleef, Maastricht UMC+

\section{Co-promotores}

Dr. R.M.H. Roumen, MMC Eindhoven / Veldhoven

Dr. M.R.M. Scheltinga, MMC Eindhoven / Veldhoven

\section{Manuscriptcommissie}

Prof. dr. N.D. Bouvy, Maastricht UMC+ (voorzitter)

Prof. dr. F.J.P.M. Huygen, Erasmus MC Rotterdam

Prof. dr. J.F.M. Metsemakers, Maastricht University

Prof. dr. J.J. van Overbeeke, Maastricht UMC+

Prof. dr. A.J.P.M. Smout, AMC Amsterdam 


\section{Table of contents}

\section{Chapter 1 Introduction, aims and outline of the thesis}

Chapter 2 Construction and validation of a questionnaire distinguishing a chronic abdominal wall pain syndrome from irritable bowel syndrome. Frontline Gastroenterology 2012; 3: 288-294

Chapter 3 Chronic abdominal wall pain misdiagnosed as functional abdominal pain. Journal of the American Board of Family Medicine 2013; 26: 738-744

Chapter 4 Incidence of abdominal pain due to the anterior cutaneous nerve entrapment syndrome in an emergency department. Scandinavian Journal of Trauma, Resuscitation and Emergency Medicine; 2015;

23: 19

Chapter 5 Anterior cutaneous nerve entrapment syndrome and the avoidable economic burden. (submitted)

Chapter 6 A double-blind, randomized, controlled trial on surgery for chronic abdominal pain due to anterior cutaneous nerve entrapment syndrome. Annals of Surgery. 2013; 257(5): 845-9

Chapter 7 Long-term success rates after an anterior neurectomy in patients with an abdominal cutaneous nerve entrapment syndrome. Surgery 2015; 157(1): 137-43 nerve entrapment syndrome. World Journal of Surgery. 2014; 38(12): 3105-11

Chapter 9 Summarizing discussion, conclusions and future perspectives

Chapter 11 Samenvatting (Dutch summary)

Dankwoord

List of publicatons

Curriculum vitae 


\section{Chapter 1}

Introduction, aims and outline of the thesis 


\section{Introduction}

"Neuralgia of the nerves which supply the abdominal wall is a subject which has never received merited recognition in medical literature. It is an exceedingly common affection and failure to recognize its presence inevitably leads to erroneous diagnoses and results often in futile operations." John B. Carnett, an American Surgeon, started his 1926 article "Intercostal Neuralgia as a Cause of Abdominal Pain and Tenderness" with this statement ${ }^{1}$. Since then, only a limited number of publications attracted attention to this pain entity ${ }^{2-6}$. Due to a lack of widespread knowledge concerning the abdominal wall, the anterior cutaneous nerve entrapment syndrome (ACNES), a characteristic abdominal wall pain syndrome, is frequently overlooked as the cause. The physicians 'preoccupation' with intra-abdominal visceral disorders is another cause for the ongoing neglect of chronic abdominal wall pain (CAWP) syndromes. This ignorance leads to unnecessary and costly consultations, multiple testing and negative abdominal surgery and may also result in frustrated patients, discouraged doctors and unhappy insurance companies ${ }^{4,7}$.

Some studies point towards a high health consumption in ACNES. However, these investigations were performed in previous eras in countries that differ from the Dutch health care system. One Canadian study reported that 418 diagnostic procedures were executed in 100 CAWP patients ${ }^{3}$. In two other studies on diagnostic testing and hospital charges before arriving at the correct diagnosis, direct medical costs were calculated to range from 680 US\$ up to a staggering 6727 US\$ per chronic abdominal wall pain (CAWP) patient ${ }^{8,9}$.

Older studies concerning ACNES mainly focused on clinical signs and treatment using pain point injections with anaesthetics, 5, 7, 10-17. However, most patients do not benefit on the long term from local anesthetics only. Some studies suggested surgical resection of end twigs of intercostal nerves, based on similar techniques performed in patients with neuropathic groin pain syndromes ${ }^{2,4}$. But until recent years, most studies in ACNES did not focus on surgical treatment.

Apart from a lack of a complete and easy to implement diagnostic and treatment protocol, there is also a lack of reliable numbers on incidence and prevalence. In daily practice, physicians continuously have to interpret a patient history and physician's examination, and combine these with estimations of the likelihood of possible causes ('differential diagnosis'). In specific situations, researchers were able to forward 'educated guesses' on ACNES incidence. For instance, approximately $1 \%$ of referrals to general surgeons were related to abdominal wall pain syndromes ${ }^{16}$. Furthermore, abdominal wall pain was diagnosed in approximately $15 \%$ of referrals for nonspecific abdominal pain in one outpatient pain clinic $^{18}$. Carnett claimed to have seen three patients per week with this diagnosis, and Applegate evaluated 1-2 patients with this diagnosis in every 150 patients in his family practice ${ }^{1,7}$. These subjective observations suggest a substantial incidence. However, such estimations are outdated and most were level five evidence rather than structurally studied incidence calculations.

There is still a lot to learn in the world of ACNES.

\section{Anatomy}

The thoraco-abdominal nerves which end up as cutaneous nerves are anchored at 5 points (Figure 1): 1) the spinal cord; 2) the point at which the posterior branch originates; 3) the point at which the lateral branch originates; 4) the point at which the anterior branch makes a nearly $90^{\circ}$ turn to enter the rectus channel; 5) and skin.

Figure 1 Course and distribution of an intercostal nerve at the abdominal level in a transverse section

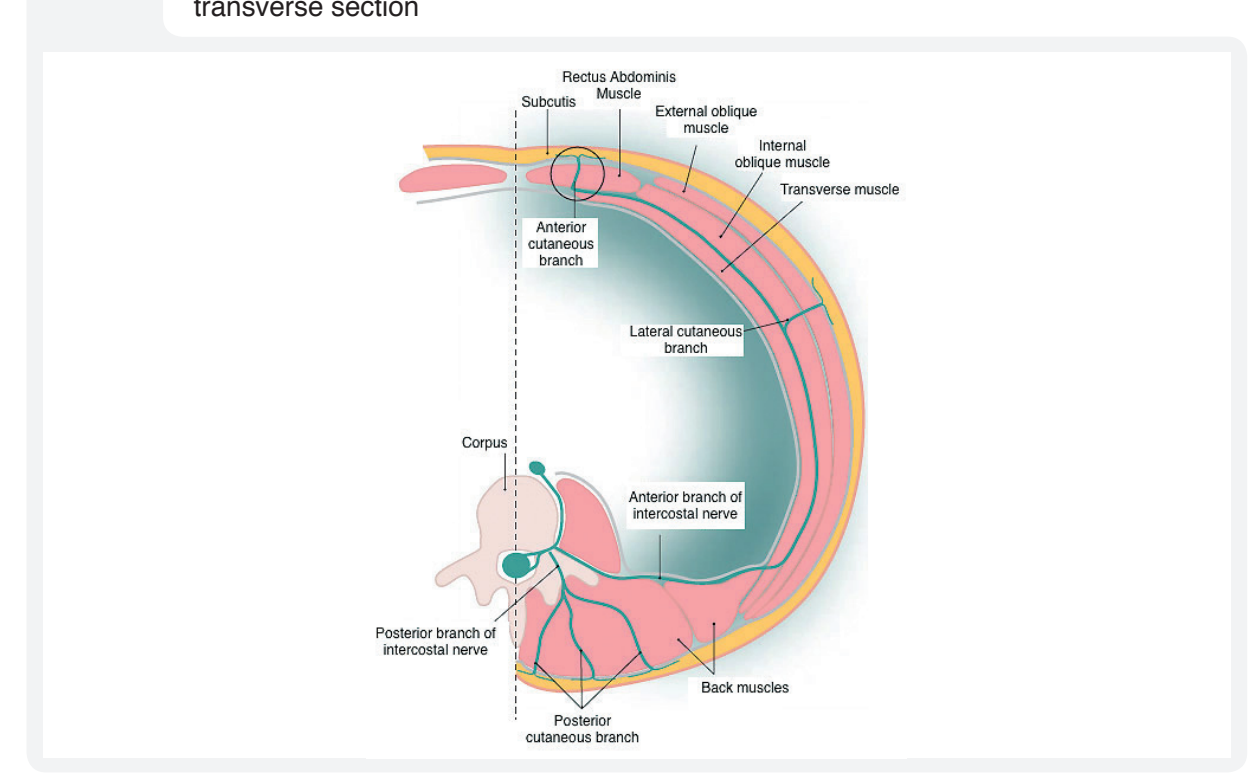


The intercostal nerves pass infero-anteriorly from the intercostal spaces and run in the neurovascular plane between the internal oblique and the transverse abdominal muscles to supply the abdominal skin and muscles. The lateral cutaneous branches emerge from the musculature of the anterolateral wall to enter the subcutaneous tissue along the anterior axillary line, whereas the anterior abdominal cutaneous branches pierce the rectus sheath to enter the subcutaneous tissue a short distance from the median plane. Skin and muscles of the anterolateral wall are mainly supplied by the following anterolateral branches of thoraco-abdominal nerve(s) (figure 2):

- $\quad$ T7 - T9 supply the skin superior to the umbilicus.

- T10 innervates the skin around the umbilicus.

- T11, plus the cutaneous branches of the subcostal (T12), iliohypogastric, and ilioinguinal (L1), supply the skin inferior to the umbilicus.

During their course through the abdominal wall, the thoraco-abdominal, subcostal, and iliohypogastric nerves inter-communicate extensively ${ }^{19}$.

\section{Figure 2 Course of thoracic intercostal nerves and their distribution over the abdominal} and inguinal region, anterior view

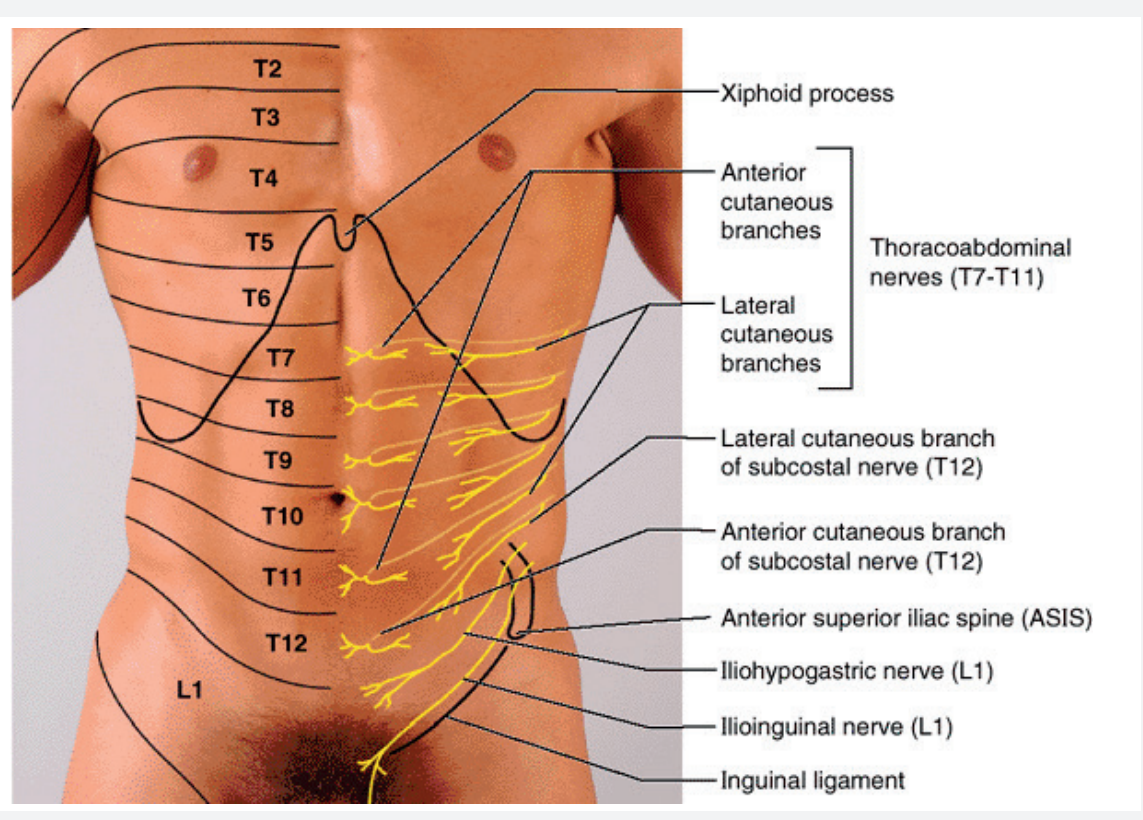

Pathophysiology \& Etiology

Peripheral nerve entrapment may occur at anatomic sites where the nerve changes direction to enter a fibrous or osseofibrous tunnel, or where the nerve passes over a fibrous or muscular band $\mathrm{d}^{7}$. At the point where an intercostal nerve penetrates through the rectus muscle (nr. 4, figure 1), a fibrous ring provides a smooth surface through which this bundle can slide. Just anterior to this ring, a hiatus in the rectus aponeurosis facilitates the passage of the anterior branch of the intercostal nerve. Localized compression at the level of this ring and subsequently nerve ischemia is hypothesized to cause a typical ACNES pain. It is thought that too much pressure from deep tissues or pulling forces from superficial structures cause the neurovascular bundle to be pushed against the ring leading to irritation and swelling of the bundle and herniation thereafter ${ }^{7}$.

Several situations can trigger the onset of ACNES or may exacerbate a current situation. Increased abdominal pressure due to 'acute' moments (coughing, sneezing, squeezing) or an abdomen diameter increase due to any cause (pregnancy 9\%) will put the nerves under greater tension. Scar tissue or sutures can directly compress the nerve or place it under traction ${ }^{20,21}$. Pulling the abdominal wall during surgery can also disturb anatomical structures (20\%). Furthermore, increased abdominal muscle use due to sports (7\%), heavy lifting or sudden movements can elicit the pain (3\%). Hypothetically, increased muscle tensing is an important underlying cause in ACNES patients who do not remember a triggering event $(54 \%)^{22}$. On the other hand, little is known about the segmental background of this type of pain syndrome ${ }^{23}$.

Clinical presentation, diagnosis and management

In patients suffering from ACNES, pain is dominant. Pain is mostly severe (VAS 7-8 on a $0-10$ scale) and is characterized as sharp or burning ${ }^{22,24}$. Maximal abdominal tenderness is usually located on a patient-characteristic spot within lateral boundaries of the rectus abdominis muscle. Onset of pain is often acute and usually worsens over time. In most patients, the pain is continuous with exacerbations. Provoking factors are certain poses (sitting, bending, laying on affected side), and activity in general. Some patients report socalled 'pseudo-visceral' complaints. Abdominal swelling, nausea, bloating and alterations in stool or increased ructus may be experienced. These nonspecific complaints may be misleading for consulting doctors, and an abdominal wall pain diagnosis is therefore easily missed. 
As ACNES is generally considered a typical clinical syndrome, physical examination is a most crucial step in the diagnosis. However, a diagnostic gold standard is currently lacking. The diagnosis is established by a set of common signs and symptoms. A constant site of abdominal tenderness with a small ( $<2 \mathrm{~cm} 2$, 'finger tip') area of maximal intensity situated is located at the level of the rectus abdominis muscle. Tenderness increases when these abdominal muscles are tensed while the patient lifts the head (Carnett's test). Local sensory disturbances of the skin are frequently present (80-90\%). By squeezing an overlying skin fold containing the patient's skin and subcutaneous fat between thumb and index finger, the area of interest very much hurts compared to the contralateral side ('pinch test').

Laboratory analysis and imaging are almost always normal in an ACNES patient if performed after history taking and the physical examination (for example on an emergency department). If the diagnosis ACNES is deemed likely, the physician may perform a local pain point infiltration using lidocain of bupivacaine as a means to confirm the diagnosis. A regimen of consecutive local pain point injections is effective in some one-thirds of patients with $\mathrm{ACNES}^{22,24}$. Surgical neurectomy is worthwhile in approximately twothirds of the injection regimen-refractory patients. In total, eighty percent of an ACNES population reported total or substantial pain relief on after a median 18 months followup following this approach ${ }^{22}$. These beneficial success rates strongly support this stepwise treatment protocol. However, individual treatment steps were hitherto not prospectively studied whereas long-term results are largely unknown.

\section{General aim of thesis}

To study epidemiology and outcome of treatment strategies in populations who were diagnosed with the anterior cutaneous nerve entrapment syndrome (ACNES).

\section{Specific aims}

1. To develop a simple tool for the identification of patients with abdominal wall pain syndromes such as ACNES among populations who are diagnosed with functional abdominal pain.

2. To determine ACNES prevalence in a functional abdominal pain population.

3. To study the incidence of acute ACNES in an emergency department.

4. To describe the socio-economic consequences in a population with ACNES.

5. To validate and to assess long-term results of a surgical neurectomy in an operated ACNES population.

6. To assess efficacy of remedial surgery after a failed neurectomy in ACNES patients as a means to introduce a next step in the treatment protocol.

\section{Outline of thesis}

An irritable bowel syndrome (IBS) population is heterogeneous, harboring a variety of abdominal symptoms (and possibly, syndromes). Therefore, IBS is often termed a 'diagnosis of exclusion. Chronic abdominal wall pain (CAWP) is a poorly recognized entity and is frequently caused by the anterior cutaneous nerve entrapment syndrome (ACNES). Some patients may be misdiagnosed because IBS and CAWP do share symptoms. In chapter 2 , construction and validation of a novel 18 -item questionnaire tool designed to distinguish patients with CAWP syndromes (including ACNES) from patients with IBS is introduced.

Most IBS patients are treated in a primary care environment. In chapter 3, the presence of CAWP syndromes (in particular ACNES) in 4 IBS cohorts in a primary care setting around the Netherlands is investigated using the validated diagnostic, 18-item abdominal wall pain questionnaire.

Most patients who are ultimately diagnosed with chronic ACNES have suffered from this entity for an extensive period of time. However, most patients report visits to an emergency department in an earlier phase of the illness. Awareness in an emergency 
department may be suboptimal. In chapter 4, the incidence of ACNES in the emergency department of a Dutch teaching hospital is investigated.

Health care consumption in ACNES is believed to be high due to an on-going neglect among physicians combined with the severity of the neuropathic pain. In chapter 5, direct medical and indirect non-medical cost in the year before the diagnosis ACNES in a cohort of 94 patients is described.

In 2011, an effective treatment regimen consisting of injection treatment followed by an anterior neurectomy in patients insufficiently responding to an injection regimen was reported in 139 patients ${ }^{22}$. The beneficial effect of a surgical neurectomy was, however, not yet demonstrated in a randomized fashion. In chapter 6 , results of a double-blind, randomized, controlled trial on surgery for abdominal pain due to ACNES are reported.

Follow up studies on the long-term efficacy of an anterior neurectomy were never performed. Chapter 7 describes long-term success rates of surgery in a large ACNES population. Although successful in most patients, this treatment regimen is not longterm beneficial in all. The efficacy of secondary surgery including re-exploration or a posterior neurectomy in patients reporting recurrent pain after initially successful surgery or following an immediately failed anterior neurectomy is described in chapter $\mathbf{8}$.

A summarizing discussion and future perspectives are provided in chapter 9. Since the beginning of 2014 a valorisation addendum is required according to the doctoral regulations. In chapter 10, the valorisation of this thesis is described. In chapter 11, a Dutch summary is provided. Acknowledgements, list of publications and a curriculum vitae of the author are provided at the end of this thesis.

\section{References}

1. Carnett J. Intercostal neuralgia as a cause of abdominal pain and tenderness. Surg Gynecol Obstet. 1926; 42: 8.

2. Applegate WV. Abdominal cutaneous nerve entrapment syndrome. Surgery. 1972; 71: 118-124.

3. Hershfield NB. The abdominal wall. A frequently overlooked source of abdominal pain. J Clin Gastroenterol. 1992; 14: 199-202.

4. Srinivasan R, Greenbaum DS. Chronic abdominal wall pain: a frequently overlooked problem. Practical approach to diagnosis and management. Am J Gastroenterol. 2002; 97: 824-830.

5. Suleiman S, Johnston DE. The abdominal wall: an overlooked source of pain. Am Fam Physician. 2001; 64: 431-438.

6. Roumen RM, Scheltinga MR. [Abdominal intercostal neuralgia: a forgotten cause of abdominal pain]. Ned Tijdschr Geneeskd. 2006; 150: 1909-1915.

7. Applegate WV. Abdominal Cutaneous Nerve Entrapment Syndrome (ACNES): A Commonly Overlooked Cause of Abdominal Pain. Perm J. 2002; 6: 20-27.

8. Greenbaum DS, Greenbaum RB, Joseph JG, et al. Chronic abdominal wall pain. Diagnostic validity and costs. Dig Dis Sci. 1994; 39: 1935-1941.

9. Thompson C, Goodman R, Rowe WA. Abdominal wall syndrome, a costly diagnosis of exclusion. Gastroenterol. 2001; 120: A637.

10. Ranger I, Mehta M, Pennington M. Abdominal wall pain due to nerve entrapment. Practitioner. 1971; 206: 791-792.

11. Ashby EC. Abdominal pain of spinal origin. Value of intercostal block. Ann R Coll Surg Engl. 1977; 59: 242-246.

12. Thomson H, Francis DM. Abdominal-wall tenderness: A useful sign in the acute abdomen. Lancet. 1977; 2: 1053-1054.

13. Tung AS, Tenicela R, Giovanitti J. Rectus abdominis nerve entrapment syndrome. Jama. 1978; 240: 738-739.

14. Gallegos NC, Hobsley M. Recognition and treatment of abdominal wall pain. J R Soc Med. 1989; 82: 343-344.

15. Abdominal wall tenderness test: could Carnett cut costs? Lancet. 1991; 337: 1134

16. Thomson WH, Dawes RF, Carter SS. Abdominal wall tenderness: a useful sign in chronic abdominal pain. Br J Surg. 1991; 78: 223-225. 
17. Sharpstone D, Colin-Jones DG. Chronic, non-visceral abdominal pain. Gut. 1994; 35: 833836.

18. Hall PN, Lee AP. Rectus nerve entrapment causing abdominal pain. Br J Surg. 1988; 75: 917.

19. Moore KL, Dalley AF. Clinically orientated anatomy. Vol. 5. 2005: Lippincott Williams \& Wilkins.

20. Loos MJ, Scheltinga MR, Mulders LG, et al. The Pfannenstiel incision as a source of chronic pain. Obstet Gynecol. 2008; 111: 839-846.

21. Gallegos NC, Hobsley M. Abdominal wall pain: an alternative diagnosis. Br J Surg. 1990; 77: 1167-1170.

22. Boelens OB, Scheltinga MR, Houterman S, et al. Management of anterior cutaneous nerve entrapment syndrome in a cohort of 139 patients. Ann Surg. 2011; 254: 1054-1058.

23. Lewis T, Kellgren JH. Observations relating referred pain, visceromotor reflexes and other associated phenomena. Clin Sci. 1939; 4.

24. Boelens OB, Scheltinga MR, Houterman S, et al. Randomized clinical trial of trigger point infiltration with lidocaine to diagnose anterior cutaneous nerve entrapment syndrome. Br J Surg. 2013; 100: 217-221. 


\section{Chapter 2}

Construction and validation of a questionnaire distinguishing a chronic abdominal wall pain syndrome from irritable bowel syndrome

Tijmen van Assen

Oliver B. Boelens

Jan T. Kamphuis

Marc R. Scheltinga

Rudi M. Roumen 


\section{Abstract}

Objective

The irritable bowel syndrome (IBS) population is heterogeneous, harbouring a variety of abdominal symptoms. Therefore, IBS is often termed a 'diagnosis of exclusion. Chronic abdominal wall pain (CAWP) is a poorly recognized entity, frequently caused by the anterior cutaneous nerve entrapment syndrome (ACNES). Some patients may be misdiagnosed because IBS and CAWP share symptoms. Aim of this study was to construct and validate a questionnaire to distinguish patients with CAWP (including ACNES) patients with IBS.

\section{Design}

A questionnaire was designed of 17 ACNES characteristic items obtained from ACNES patients $(n=33)$ and expert opinion of two specialized surgeons. Eleven IBSrelated items ('Rome III' criteria) were added leading to a questionnaire containing 28 items. This was validated in a 'gold standard' ACNES group (successfully operated ACNES patients, $\mathrm{n}=68$ ) and a 'prospective' IBS group $(\mathrm{n}=64)$ as well as in a 'prospective' ACNES group $(n=47)$. Distinctive power of individual items was analyzed by $\chi 2$. Reliability was tested with Crohnbach's $\alpha$. ROC curve was used to determine cut-off values.

\section{Results}

Eighteen of 28 items were significantly distinctive $(\mathrm{p}<0.01)$ between ACNES and IBS patients leading to an 18-point ACNES score with good internal consistency $(\alpha=0.85)$. Cut-off value of 10 points resulted in $94 \%$ sensitivity, $92 \%$ specificity and areas under the curve (AUC) of 0.98. Evaluation of the prospective ACNES group led to $85 \%$ sensitivity, $92 \%$ specificity and AUC 0.95 indicating high discriminative properties of the questionnaire.

Conclusions

This novel questionnaire may be useful and valid as a simple tool distinguishing patients harbouring a CAWP syndrome from those having IBS.

\section{Objective}

Irritable Bowel Syndrome (IBS) is a syndrome that is characterized by spells of abdominal discomfort and pain. According to the Rome III criteria, the diagnosis is based on clinical symptoms in the absence of structural or tissue abnormalities. IBS patients are defined to report abdominal complaints at least 3 days per month in the previous 3 months. Moreover, the diagnosis is probable if two or more of the following criteria are fulfilled. The onset is associated with a change in frequency and/or appearance of stool whereas symptoms are attenuated after defecation ${ }^{1}$.

Due to the descriptive and general character of these Rome III criteria, a current IBS patient population is heterogeneous harbouring a broad variety of abdominal symptoms. As a consequence, IBS is often termed a 'diagnosis of exclusion'2. Estimations on prevalence of IBS in The Netherlands may vary from 5-20\% in an average community. Approximately 1 of 3 IBS patients requests medical help ${ }^{3-6}$. Consequently, some half a million Dutch people are calculated to require treatment for IBS.

Chronic abdominal pain (CAP) is usually thought to arise from a diseased viscus. However, CAP may also be caused by an abnormality situated in the wall of the abdomen. A common cause of this latter category is the anterior cutaneous nerve entrapment syndrome (ACNES). ACNES is caused by entrapment of branches of intercostal nerves (Th. 8-12) causing a severe chronic abdominal pain. Myofascial pain and radiculopathy are considered less frequent causes of abdominal wall pain ${ }^{7,8}$. As a tertiary referral centre, our research group is especially interested in CAP due to ACNES. Carnett's statement some 85 years ago that 'the ACNES problem never received merited recognition in medical literature' still holds true as of today ${ }^{9-15}$. The diagnosis ACNES is based on the finding of a constant site of tenderness that is superficially located with a small area of maximal tenderness that can be localized with a fingertip (trigger point). The tenderness is invariably increased after abdominal muscle tensing using the Carnett's test. Moreover a small area of somatosensory alterations surrounding the trigger point is often found in ACNES possibly allowing for discrimination between a myofascial cause and a radicular cause of the pain ${ }^{9}, 11,16-19$. The diagnosis ACNES can be substantiated by a subfascial injection of $1 \%$ lidocain, typically leading to significant pain reduction after 15 to 20 minutes $^{20}$. Abnormalities in blood and organ tissues are practically always absent in ACNES patients. Recently, our group evaluated a diagnostic work up protocol and treatment regimen in 139 consecutive patients with suspected ACNES. Local trigger 
point injections appeared long term effective in one-third of the patients. Surgical neurectomy was effective in about two-thirds of the refractory patients. Some $80 \%$ of the entire ACNES population reported total or substantial pain relief on the long term ${ }^{20}$.

As our experience with unravelling the diagnosis in patients with CAWP is growing, we were confronted with some patients harbouring ACNES although previously diagnosed with IBS. Aim of the present study was to develop and validate a questionnaire that may be used to differentiate between IBS and CAWP. We hypothesized that the novel questionnaire may allow a discrimination between various patient populations having CAP.

\section{Design}

\section{Setting}

The study was coordinated by members of the Surgical department and was performed in outpatient departments of Gastroenterology and Surgery of Máxima Medical Centre (MMC) in Veldhoven, The Netherlands. The MMC is an 865-bed community hospital serving a population of approximately $350.000-400.000$. It is a tertiary referral centre for abdominal wall and groin pain syndromes ${ }^{20-27}$.

\section{Development of questionnaire}

The preliminary questionnaire was composed in November and December 2010 by input from two focus groups and the input from IBS literature. The first focus group consisted of 70 ACNES patients that had undergone successful surgery between 2003 and 2008 . They were randomly selected to contribute to the development of the questionnaire. They were requested via an accompanying letter to describe in detail all of the symptoms they had experienced in the period prior to surgery. The second focus group consisted of two surgeons skilled in diagnosing and treating ACNES. They were invited to report typical symptoms from a professional point of view (RR and MS). They both independently provided a list containing what they considered typical complaints reflecting the syndrome of ACNES. Data obtained from both focus groups were used to identify the most ACNES sensitive questions that were subsequently used in the questionnaire. This was complemented by inserting typical IBS related questions obtained from three different sources (Birmingham IBS questionnaire, Rome III criteria, Dutch IBS interest group ${ }^{28-30}$. All questions were closed-ended, dichotomous ('yes', 'no') or 4-point Likertscale questions ('mostly', 'regularly', 'sometimes', 'never').

\section{Validation of questionnaire}

Between February and July 2011 three patient populations were utilized for validation. The first population consisted of a group of ACNES patients that had demonstrated a favourable response to surgery defined as a $>50 \%$ reduction in pain (measured by Visual Analogue Scale (VAS)) or a $>2$ point reduction on a 5 point Verbal Rating scale (VRS) between 2003 and 2011 ('gold standard' ACNES group). This definition was considered 'gold standard' in the absence of an alternative one. To obtain this information, a short survey investigating complaints before and after surgery was added to the questionnaire. This first validation population partially overlapped with the population used for questionnaire development. They completed the questionnaire by paper or by the use of the web-based questionnaire from the ThesisTools ${ }^{\circledR}$ system. A reminder was sent after four weeks if the participant remained unresponsive. Inclusion ended 3 months after the first contact. Responds with more than one missing item were excluded from analysis.

To overcome a possible shortcoming associated with the retrospective character of these treated ACNES patients, a second population of newly diagnosed ACNES patient that presented to the surgical outpatient department was also included for validation purposes ('prospective' ACNES group). They were diagnosed between May and July 2011 and completed the questionnaire prior to visiting the specialist in the outpatient department. The diagnosis ACNES was confirmed in all individuals by one of the 2 expert surgeons.

The third population used for validation consisted of a cohort of consecutive patients with a suspected diagnosis of IBS presenting at the gastroenterological outpatient department ('prospective' IBS group). Patients were prospectively included between April and May 2011 and completed the web-based survey. Four weeks after the inclusion of these new IBS-patients, diagnosis based on the Rome III criteria was re-checked for confirmation

\section{Calculations}

Data analysis was performed using SPSS 18.0 for MacOS X. During the validation phase, two different steps were executed. First, Likert-scale questions were dichotomised before analysis. 'Mostly' and 'regularly' were interpreted as a positive answer, where 'sometimes' 
and 'never' were interpreted as a negative answer. Subsequently, the distinctive power of each of both ACNES and IBS items of the questionnaire were measured using chi-square $\left(\mathrm{chi}^{2}\right)$ analysis. A $<0.01$ chi-square cut-off point was used. Non-distinctive questions $\left(\mathrm{chi}^{2}>0.01\right)$ were excluded from the questionnaire. Reliability of the questionnaire was determined using Crohnbachs- $\alpha$. An $\alpha>0.9$ is considered excellent, $\alpha>0.8$ is good, $\alpha>0.7$ acceptable, $\alpha>0.6$ is questionable, $\alpha>0.5$ is poor, and $\alpha<0.5$ is unacceptable ${ }^{31}$.

A second step was to develop a scoring algorithm for the questionnaire. Two scenarios were evaluated using results from the gold standard ACNES group and from the prospective IBS group. In the first scenario, a simple algorithm was evaluated contributing 1 point to each question. In the second scenario, points were given based on the distinctive character of a question. Grade of distinctive character was determined by the risk rate of a question. One point was given for a risk rate between 1 and 2.5, two points for a risk rate between 2.5 and 5, and three points for questions with a risk rate $>5$. Each ACNES related question received point(s) for a positive answer whereas an IBS related question scored point(s) for a negative answer. Patients with a high score were likely suffering from ACNES, whereas low scores were attained by a typical IBS patient. Cut-off values and discriminating values (sensitivity and specificity) were tested in both ACNES groups using a ROC-curve. In the end, a final scoring algorithm was constructed.

\section{Results}

\section{Development of questionnaire}

The response of 33 ACNES patients (response rate 47\%) combined with the opinion of both surgeons led to 17 questions that were deemed sensitive and distinctive for ACNES. A total of 11 IBS related questions was added resulting in a preliminary 28 -item questionnaire. ACNES and IBS questions were sequenced in random order. Twenty-two of the 28 questions investigated pain (course over time $n=3$, type $n=5$, localization $n=7$, provocative factors $n=7$ ) whereas six questions were concerned with other symptoms. Twelve questions were dichotomous, whereas the remaining 16 were 4 -point Likert-scale questions.

\section{Validation of questionnaire}

Subjects

In the gold standard ACNES group, a total of 101 responses was obtained from 130 ACNES patients (response rate $78 \%$ ). Of these 101 patients, 73 subjects were successfully treated (success rate 72\%). As 5 responders were excluded due to missing items, answers of 68 questionnaires were eligible for analysis (female $81 \%$, age $48 \pm 19$ yr.). In the prospective ACNES group, 83 patients suspected of ACNES and visiting the outdoor department of surgery were eligible for validation. As 36 patients were excluded (questionnaire completed after rather than before diagnosis $(n=21)$, uncertain moment of questioning $(n=3)$, other diagnosis $(n=8)$, missing items $n=4)$, questionnaires of 47 remaining subjects with the diagnosis of ACNES were eligible for analysis (female $79 \%$, age $46 \pm 17 \mathrm{yr}$ ). In the prospective IBS group, a population of 66 subjects was drafted from the IBS-group. As 2 patients were excluded (haemorrhoids $n=1$, ACNES $n=1$ ), a cohort of 64 IBS subjects was eligible for analysis (female $69 \%$, age $50 \pm 6$ yr., Table 1 ).

\begin{tabular}{lcccc} 
Table 1 & $\begin{array}{c}\text { Demographic data of validation groups } \\
\text { Gold st. ACNES } \\
(\mathbf{n}=101)\end{array}$ & $\begin{array}{c}\text { Pros. ACNES } \\
(\mathbf{n = 8 3})\end{array}$ & $\begin{array}{c}\text { Prosp. IBS } \\
(\mathbf{n = 6 6 )}\end{array}$ & $\begin{array}{c}\text { p-value } \\
\text { (ACNES vs. IBS) }\end{array}$ \\
\hline Excluded (N) & 33 & 36 & 2 & \\
Eligible for analysis (N) & 68 & 47 & 64 & \\
Male / Female (\%) & $19 / 81$ & $21 / 79$ & $31 / 69$ & 0.09 \\
Age (SD) & $48(19)$ & $46(17)$ & $50(18)$ & 0.38 \\
\hline
\end{tabular}

(gold st. $=$ gold standard, prosp. $=$ prospective)

Definitive form of questionnaire

Some 18 of 28 items showed sufficient discriminative properties between gold standard ACNES group and prospective IBS patient group $\left(\mathrm{chi}^{2}<0.01\right)$. This set of 18 items included 11 ACNES and 7 IBS questions. In this final set of questions 14 questions investigated pain (course over time $n=1$, type $n=3$, localization $n=5$, provocative factors $n=7$ ) whereas four questions concerned additional complaints. The 18 questions with distinctive character had a good internal consistency $(\alpha=0.85$ ). These properties allowed for calculation of a total score. Table 2 shows discriminating symptoms associated with either ACNES or IBS. 
Table 2 Discriminating items in the questionnaire. Translated from Dutch

\begin{tabular}{|c|c|c|c|}
\hline & $\begin{array}{l}\text { Gold St. ACNES } \\
\mathrm{n}=68\end{array}$ & $\begin{array}{c}\text { Prosp. } \\
\text { IBS } \\
\mathrm{n}=64 \\
\end{array}$ & $\begin{array}{c}\mathrm{Chi}^{2} \\
\text { (p-val.) }\end{array}$ \\
\hline ACNES-items: Positive & \multicolumn{2}{|c|}{$\begin{array}{l}\text { Yes or } \\
\text { Mostly / Regularly (\%) }\end{array}$} & \\
\hline Pain is sharp (cutting) & 81 & 36 & $<0.001$ \\
\hline For me PAIN dominates over DISCOMFORT & 83 & 45 & $<0.001$ \\
\hline Pain is always located on the same spot(s) & 94 & 73 & 0.001 \\
\hline Pain is located just lateral to the midline of the abdomen & 80 & 45 & $<0.001$ \\
\hline $\begin{array}{l}\text { The most intense pain can be localized by the tip of one } \\
\text { finger }\end{array}$ & 91 & 48 & $<0.001$ \\
\hline I believe the pain is originated just beneath the skin & 57 & 20 & $<0.001$ \\
\hline $\begin{array}{l}\text { Pain is provoked by daily activities (e.g. walking, sitting, } \\
\text { cycling, bending) }\end{array}$ & 80 & 33 & $<0.001$ \\
\hline Lying on the affected side aggravates the pain & 57 & 13 & $<0.001$ \\
\hline Pushing on the painful spot aggravates the complaints & 85 & 38 & $<0.001$ \\
\hline Coughing, sneezing of squeezing aggravates the pain & 65 & 11 & $<0.001$ \\
\hline The painful spot feels strange, different or dull & 54 & 16 & $<0.001$ \\
\hline IBS- items: Negative & \multicolumn{2}{|c|}{$\begin{array}{l}\text { No or } \\
\text { Sometimes / Never (\%) }\end{array}$} & \\
\hline $\begin{array}{l}\text { I believe the complaints originate from my gastrointestinal } \\
\text { tract }\end{array}$ & 73 & 16 & $<0.001$ \\
\hline Pain exists on different spots all over the abdomen & 86 & 53 & $<0.001$ \\
\hline $\begin{array}{l}\text { Complaints are somehow related to an altered defecation } \\
\text { pattern }\end{array}$ & 99 & 36 & $<0.001$ \\
\hline Stress provokes the complaints & 88 & 63 & 0.001 \\
\hline I experience bloating or a feeling of gas in the intestines & 73 & 23 & $<0.001$ \\
\hline $\begin{array}{l}\text { The stool has an abnormal consistency (e.g. hard and } \\
\text { small, pencil thin, loose, watery) }\end{array}$ & 76 & 33 & $<0.001$ \\
\hline $\begin{array}{l}\text { I feel urgent need for bowel movement without producing } \\
\text { stool (incomplete defecation) }\end{array}$ & 90 & 64 & $<0.001$ \\
\hline
\end{tabular}

(gold st. = gold standard, prosp. $=$ prospective)
Cut off value

Any test cut off point must be chosen on the basis of a hypothesis. As knowledge about ACNES is scarce and a minimal prevalence (some \%'s) in the IBS-group was expected, the questionnaire should have optimal identification properties. Consequently, such an identification tool needs high specificity. A 10 points cut off value provides an optimal 0.94 sensitivity, combined with 0.92 specificity.

There was a slight reduction in the prospective ACNES group, but the test characteristics were still satisfactory (table 3 ). Sensitivity was 0.85 for a 10 points cut off value in this group. Calculated ROC curves of both gold standard ACNES group and the prospective ACNES group give outstanding areas under the curve (AUC), representing an excellent diagnostic test. The area under the curve (AUC) of the gold standard group is 0.98 , and the AUC of the prospective ACNES group is 0.95 . Scores of all subjects are depicted in the combined histogram (figure 1).

Table 3 Characteristics of questionnaire with different cut off values in the prospective ACNES-group compared with the gold standard ACNES-group

\begin{tabular}{cccc} 
& $\begin{array}{c}\text { Gold Standard ACNES } \\
(\mathbf{n}=68)\end{array}$ & $\begin{array}{c}\text { Prospective ACNES } \\
(\mathbf{n}=\mathbf{4 7})\end{array}$ & $\begin{array}{c}\text { Prospective IBS } \\
(\mathbf{n}=66)\end{array}$ \\
\hline Cut-off value & Sensitivity & Sensitivity & Specificity \\
\hline 7 & 1.00 & 0.98 & 0.61 \\
8 & 0.99 & 0.94 & 0.72 \\
9 & 0.96 & 0.89 & 0.86 \\
$\mathbf{1 0}$ & $\mathbf{0 . 9 4}$ & $\mathbf{0 . 8 5}$ & $\mathbf{0 . 9 2}$ \\
11 & 0.90 & 0.75 & 0.98 \\
12 & 0.78 & 0.64 & 1.00 \\
\hline
\end{tabular}

Scoring algorithm

The first ' 1 point per item' scenario led to a minimal score of 0 and an 18 points maximum score whereas the second scenario had a maximum score of 26 points. Both scenarios were tested for sensitivity and specificity at different cut off values. As the more complicated scenario 2 conferred no beneficial qualities at the best cut off value (scenario 1: $>10$ points; sensitivity 0.94 , specificity 0.92 versus scenario 2 : $>13$ points; sensitivity 0.93 , specificity $0.95)$, the simple one-point-per-question scenario was chosen. 
Figure 1 Scores of 64 irritable bowel syndrome (IBS) patients (top figure). In the lower figure scores of 68 gold standard (Gold st.) anterior cutaneous nerve entrapment syndrom (ACNES) patients and 47 prospective (Prosp.) ACNES patients. The vertical line is the cut-off value ( $>10$ points)

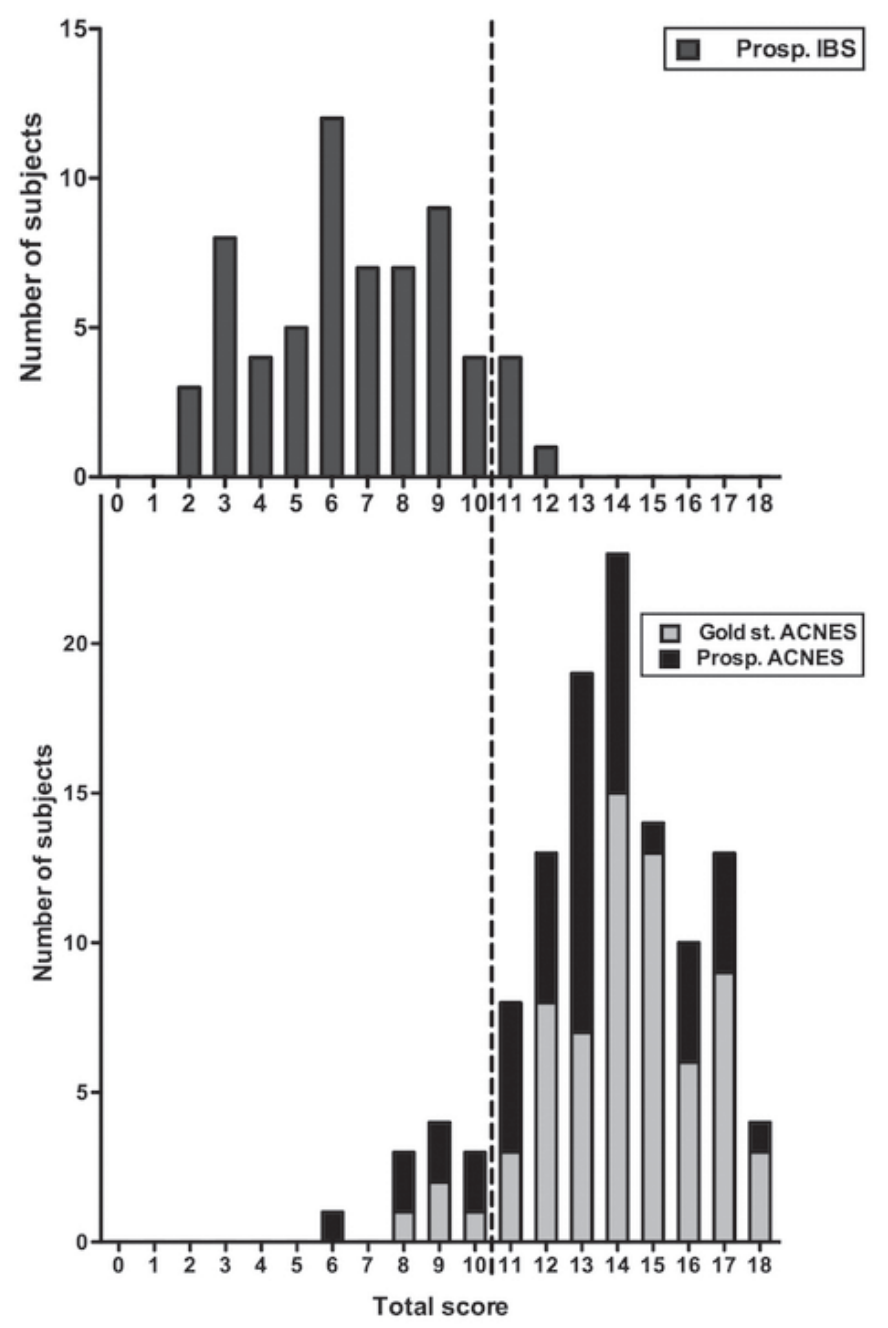

\section{Discussion}

The present study describes a stepwise development and validation of a novel diagnostic tool for the identification of potential ACNES patients within an IBS population. The results indicate that a simple 18 -item questionnaire has a 0.94 sensitivity combined with 0.92 specificity. How should these test characteristics be interpreted? Test performance of nation wide screening programmes used for breast and cervix cancer demonstrates sensitivity varying from $0.33-0.91$ combined with a $0.81-0.99$ specificity ${ }^{32-35}$. The qualifications of the present CAWP/ACNES questionnaire seem substantially better whereas its accuracy is excellent (AUC 0.98). Furthermore, a strong internal consistency $(\alpha=0.85)$ contributes to the power of the questionnaire as an identification tool.

A strong point of the present study is the validation in 2 separate populations of ACNES patients, a retrospective 'gold standard' and a 'prospective' ACNES group Answers on 4 of the definitive 18 questions appeared significantly different $\left(\mathrm{chi}^{2}<0.01\right)$ between these two groups. As 3 of these items were IBS-specific questions, it may seem that ACNES patients in the prospective group are more convinced of a visceral cause of their abdominal symptoms. These findings also suggest that information bias combined with recall bias probably played a role in the slight reduction of these test characteristics in the gold standard group compared to the prospective one.

Comparing answers of both groups of ACNES with answers derived from the IBS patients indicated that the discriminating character of the prospective ACNES group was slightly inferior. More specifically, 2 questions appeared less distinctive $\left(\mathrm{chi}^{2}>0.01\right)$ compared to its performance in the retrospective gold standard ACNES group. Both items were IBS related items ('The stool has an abnormal consistency' and 'I feel urgent need for bowel movement without producing stool (incomplete defecation)'). However, as both questions still had significant distinctive properties $(<0.05)$, they were not removed from the ultimate 18 -item questionnaire. These observations are in line with the presence of 'pseudo-visceral symptoms' that ACNES patients occasionally report ${ }^{36,37}$. The patient's conviction of a visceral origin of pain complicates the process of diagnosing ACNES in daily clinical practice.

All questions of the Dutch questionnaire were translated into the English language as depicted in table 2. Officially, linguistic validation should be performed before this questionnaire can be used in a language other than the original language. 
The current study harbours flaws. Interpretation is limited by selection bias. Although a contemporary IBS population probably includes a broad range of patients presenting to various health care providers including general practitioners, the present questionnaire is only validated with IBS-patients presenting at the gastroenterology outpatient department. It may be possible that general practitioners are confronted with a different subpopulation of alleged IBS patients presenting with a slightly different pattern of intestinal symptoms. We are currently engaged in a study investigating the test characteristics of the ACNES questionnaire in IBS patients presenting to a number of general practices.

Another flaw of the study is caused by the differential diagnosis of chronic abdominal wall pain. When the present study was designed, the authors were convinced that ACNES and CAWP were (almost) identical. In other words, it was thought that chronic abdominal wall pain was practically always caused by ACNES. The fact that some patients diagnosed with ACNES (albeit a minority) did not demonstrate altered skin sensibility urged us to assume that the differential diagnosis of CAWP harbours more entities including myofascial pain or radiculopathies. To facilitate understanding of the present study, we chose to use the terms ACNES and CAWP interchangeably.

A substantial number of patients with functional gastrointestinal disorders also report abdominal wall pain. The present questionnaire will have less discriminating power in this subpopulation. On the other hand, if the abdominal wall problems play a prominent role in the patients' pain experience, there is a reasonable chance that this individual is identified after completing the questionnaire. This is particularly true as 11 of the 18 items are obtained from ACNES patients ('ACNES/CAWP specific items'). In addition, most items (14 of 18) investigate issues of pain whereas abdominal wall syndromes are mainly characterized by pain and not by functional complains.

Despite explicit guidelines and criteria, clinicians often consider IBS a diagnosis of exclusion. Surprisingly, no guideline on IBS reported on findings obtained during a standard physical examination. On the other hand, ACNES is preferentially diagnosed after a simple but proper physical examination. A significant number of patients may be incorrectly burdened with an IBS diagnosis even more so if pseudo-visceral complaints are experienced. The present questionnaire may prove a valuable tool for the identification of patients with CAWP wrongly assumed as having IBS. The tool may be relevant for several groups of specialists including gastroenterologists and general practitioners. Moreover, also other specialists may find this questionnaire helpful in the evaluation of patients with chronic abdominal discomfort.

\section{Conclusion}

A simple 18-item questionnaire may possibly distinguish patients who potentially suffer from chronic abdominal wall pain, and in particular anterior cutaneous nerve entrapment syndrome, from a population diagnosed with irritable bowel syndrome. 


\section{References}

1. Drossman DA. Rome Foundation. [Web page]; Available from: http://www.romecriteria. org/ (accessed 03 June 2008)

2. Spiegel BM, Farid M, Esrailian E, et al. Is irritable bowel syndrome a diagnosis of exclusion?: a survey of primary care providers, gastroenterologists, and IBS experts. Am J Gastroenterol. 2010; 105: 848-858.

3. Drossman DA, Whitehead WE, Camilleri M. Irritable bowel syndrome: a technical review for practice guideline development. Gastroenterol. 1997; 112: 2120-2137.

4. Talley NJ, Weaver AL, Zinsmeister AR, et al. Onset and disappearance of gastrointestinal symptoms and functional gastrointestinal disorders. Am J Epidemiol. 1992; 136: 165-177.

5. Van der Velden J. Een nationale studie naar ziekten en verrichtingen in de huisartspraktijk. Utrecht, NIVEL. 1991.

6. Okkes I. Van Klacht naar Diagnose, Bussum: Coutinho. 1998.

7. Roumen RM, Scheltinga MR. [Abdominal intercostal neuralgia: a forgotten cause of abdominal pain]. Ned Tijdschr Geneeskd. 2006; 150: 1909-1915.

8. Srinivasan R, Greenbaum DS. Chronic abdominal wall pain: a frequently overlooked problem. Practical approach to diagnosis and management. Am J Gastroenterol. 2002; 97: $824-830$.

9. Greenbaum DS, Greenbaum RB, Joseph JG, et al. Chronic abdominal wall pain. Diagnostic validity and costs. Dig Dis Sci. 1994; 39: 1935-1941.

10. Camilleri M. Management of patients with chronic abdominal pain in clinical practice. Neurogastroenterol Motil. 2006; 18: 499-506.

11. Ranger I, Mehta M, Pennington M. Abdominal wall pain due to nerve entrapment. Practitioner. 1971; 206: 791-792.

12. Ashby EC. Abdominal pain of spinal origin. Value of intercostal block. Ann R Coll Surg Engl. 1977; 59: 242-246.

13. Nazareno J, Ponich T, Gregor J. Long-term follow-up of trigger point injections for abdominal wall pain. Can J Gastroenterol. 2005; 19: 561-565.

14. Thome J, Egeler C. Abdominal cutaneous nerve entrapment syndrome (ACNES) in a patient with a pain syndrome previously assumed to be of psychiatric origin. World J Biol Psychiatry. 2006; 7: 116-118.

15. Ivens $\mathrm{D}$, Wojciechowski $M$, Vaneerdeweg $\mathrm{W}$, et al. Abdominal cutaneous nerve entrapment syndrome after blunt abdominal trauma in an 11-year-old girl. J Pediatr Surg. 2008; 43: E19-21.
16. Carnett J. Intercostal neuralgia as a cause of abdominal pain and tenderness. 1926; 42: 8 .

17. Apllegate WV. Abdominal Cutaneous Nerve Entrapment Syndrome (ACNES): A Commonly Overlooked Cause of Abdominal Pain. Perm J. 2002; 6: 20-7.

18. McGrady EM, Marks RL. Treatment of abdominal nerve entrapment syndrome using a nerve stimulator. Ann R Coll Surg Engl. 1988; 70: 120-122.

19. Thomson WH, Dawes RF, Carter SS. Abdominal wall tenderness: a useful sign in chronic abdominal pain. Br J Surg. 1991; 78: 223-225.

20. Boelens OB, Scheltinga MR, Houterman S, et al. Management of anterior cutaneous nerve entrapment syndrome in a cohort of 139 patients. Ann Surg. 2011; 254: 1054-1058.

21. Scheltinga MR, Boelens OB, Tjon ATWE, et al. Surgery for refractory anterior cutaneous nerve entrapment syndrome (ACNES) in children. J Pediatr Surg. 2011; 46: 699-703.

22. Loos MJ, Scheltinga MR, Roumen RM. Surgical management of inguinal neuralgia after a low transverse Pfannenstiel incision. Ann Surg. 2008; 248: 880-885.

23. Loos MJ, Roumen RM, Scheltinga MR. Chronic sequelae of common elective groin hernia repair. Hernia. 2007; 11: 169-173.

24. Loos MJ, Scheltinga MR, Mulders LG, et al. The Pfannenstiel incision as a source of chronic pain. Obstet Gynecol. 2008; 111: 839-846.

25. Loos MJ, Scheltinga MR, Roumen RM. Tailored neurectomy for treatment of postherniorrhaphy inguinal neuralgia. Surgery. 2010; 147: 275-281

26. Loos MJ, Roumen RM, Scheltinga MR. Classifying post-herniorrhaphy pain syndromes following elective inguinal hernia repair. World J Surg. 2007; 31: 1760-1765; discussion 1766-1767.

27. Loos MJ, Verhagen T, Scheltinga MR, et al. A randomised controlled trial of injection therapy versus neurectomy for post-herniorrhaphy inguinal neuralgia: rationale and study design. Hernia. 2010; 14: 593-597.

28. Roalfe AK, Roberts LM, Wilson S. Evaluation of the Birmingham IBS symptom questionnaire. BMC Gastroenterol. 2008; 8: 30.

29. Beintema MLJS, Patientonderzoek PDSB, Utrecht: VWB Intermedical. 2009

30. Thompson WG. The road to rome. Gastroenterol. 2006; 130: 1552-1556.

31. Mallery G. SPSS for Windows step by step: A simple guide and reference. 11.0 update. 4 ed2003, Boston: Allyn \& Bacon. 400.

32. Kuhl CK, Schrading S, Leutner CC, et al. Mammography, breast ultrasound, and magnetic resonance imaging for surveillance of women at high familial risk for breast cancer J Clin Oncol. 2005; 23: 8469-8476. 
33. Leach MO, Boggis CR, Dixon AK, et al. Screening with magnetic resonance imaging and mammography of a UK population at high familial risk of breast cancer: a prospective multicentre cohort study (MARIBS). Lancet. 2005; 365: 1769-1778.

34. Kriege M, Brekelmans CT, Boetes C, et al. Efficacy of MRI and mammography for breastcancer screening in women with a familial or genetic predisposition. N Engl J Med. 2004; 351: 427-437.

35. Vierhout ME. Praktische Gynaecologie. Vol. 8. 2005, Houten: Bohn Stafleu van Loghem.

36. Choi YK, Chou S. Rectus syndrome. Another cause of upper abdominal pain. Reg Anesth. 1995; 20: 347-351.

37. Haynsworth RF, Jr., Noe CE. An unusual presentation of intercostal neuralgia. Anesthesiol. 1990; 73: 779-780. 


\section{Chapter 3}

Chronic abdominal wall pain misdiagnosed as functional abdominal pain

Tijmen van Assen

Jenneke W.A.J. de Jager-Kievit

Marc R. Scheltinga

Rudi M.H. Roumen 


\section{Abstract}

Introduction

The abdominal wall is often neglected as a cause of chronic abdominal pain. Aim of this study was to identify chronic abdominal wall pain syndromes, such as anterior cutaneous nerve entrapment syndrome (ACNES), in a patient population diagnosed with functional abdominal pain, including irritable bowel syndrome.

\section{Methods}

In this cross-sectional analysis, 4 Dutch primary care practices employing physicians who were unfamiliar with ACNES were selected. 535 patients $\geq 18$ years old who were registered with a functional abdominal pain diagnosis were approached to complete a validated 18-item questionnaire (maximum 18 points). Responders who scored at least the 10-point cutoff value (sensitivity, 0.94; specificity, 0.92) underwent a diagnostic evaluation to establish their final diagnosis. Main outcome was the presence and prevalence of ACNES in symptomatic patients diagnosed with functional abdominal pain.

\section{Results}

Of 535 patients, 304 (57\%) responded; 167 subjects (31\%) reporting symptoms completed the questionnaire. Of 23 patients who scored above the cutoff value, 18 were available for diagnostic evaluation. In half of these subjects $(n=9)$ functional abdominal pain was confirmed. The other 9 patients were suffering from abdominal wall pain syndrome, 6 of whom were diagnosed with ACNES (3.6\% prevalence rate; $95 \%$ confidence interval, 1.7-7.6), whereas the remaining 3 harbored a painful lipoma, an abdominal herniation, and a painful scar.

\section{Conclusions}

A clinically relevant portion of patients previously diagnosed with functional abdominal pain syndrome in a primary care environment suffers from an abdominal wall pain syndrome such as ACNES.

\section{Introduction}

Diseased organs are frequently identified as the source of chronic abdominal discomfort and pain. If history taking, physical examination and diagnostic evaluations are not abnormal, a common functional abdominal pain diagnosis such as the Irritable Bowel Syndrome (IBS, prevalence of $10-15 \%$ in Europe and North America ${ }^{1}$ ) may be considered $^{2}$. In contrast, the abdominal wall and the pelvic floor are often neglected as a cause of chronic abdominal discomfort and pain. Apart from ignorance and viscera preoccupation by physicians, complaints mimicking a problem of visceral origin ('pseudo-visceral') reported by patients also frustrate a diagnosis of chronic abdominal wall pain (CAWP) $)^{3-5}$

Myofascial pain and radiculopathy are rare examples of a CAWP syndrome. However, CAWP is commonly caused by entrapment of an anterior cutaneous branch of one or more thoracic intercostal nerves ${ }^{6}$. Patients with this anterior cutaneous nerve entrapment syndrome (ACNES) have frequently undergone repetitive diagnostic evaluations. Unfortunately, these tests are almost always inconclusive leading to a disillusioned patient and, even more so, a disappointed doctor ${ }^{3,6-14}$. As a consequence, patients with CAWP often receive a diagnosis of exclusion such as IBS ${ }^{2,15}$.

Primary aim of the present study was to identify patients with CAWP syndromes (in particular ACNES) in an IBS cohort using a validated diagnostic 18 item abdominal wall pain questionnaire in a primary care setting. We hypothesised that a portion of the IBS population was incorrectly diagnosed and actually suffered from an abdominal wall pain syndrome. As a consequence of this study, awareness may be increased among primary care physicians. 


\section{Methods}

This study was a cross-sectional analysis of primary care patients with IBS and was coordinated by members of the Surgical Department of the Máxima Medical Center (MMC) in Veldhoven/Eindhoven, The Netherlands. The MMC Medical Ethical Committee approved its specifics (no. NL36256.015.11). Sample size estimation was performed by a power analysis based on a common sense estimated prevalence prediction of $5 \%$ of CAWP patients within an alleged IBS cohort, resulting in 292 subjects needed to screen ${ }^{16}$. An estimated response rate of $60 \%$ would lead to a number needed to approach of 487 subjects.

\section{Figure 1 Typical position of ACNES trigger points. At these points the anterior twigs of the intercostal nerves penetrate the rectus abdominal muscle (T7-T12). The level of the umbilicus corresponds with intercostal nerve T10. The most typical site is T11 on the} right side.

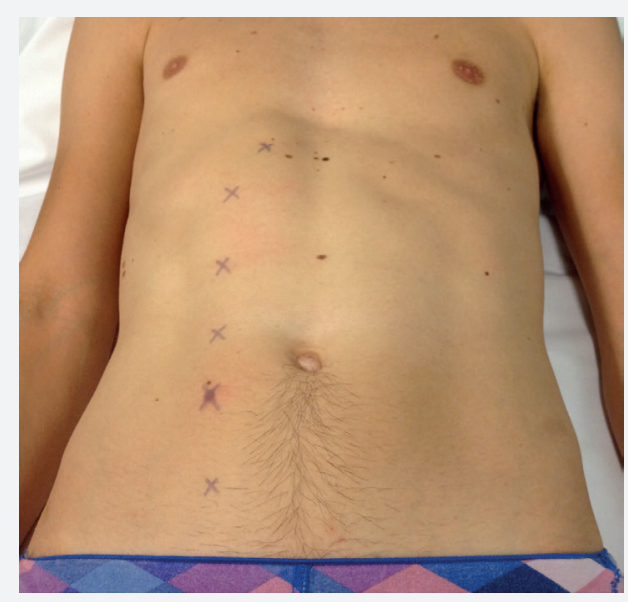

Selection of a population with a high risk on a CAWP syndrome within the IBS population was performed using a recently developed and validated 18 item ACNES questionnaire (18-pAQ, figure 3) ${ }^{17}$. This $18-\mathrm{pAQ}$ was found to clearly distinct between abdominal wall pain syndromes such as ACNES and IBS. Scores of the 18-pAQ may range from $0-18$ points. A 10 points cut-off value was found to have an optimal $94 \%$ sensitivity and $92 \%$ specificity.
As our surgical department has a long-standing interest in abdominal wall pain syndromes that is frequently communicated to neighbouring primary care physicians, it was decided to perform the study with more distant practices. Therefore, 4 Dutch primary care practices located beyond twenty kilometres from the MMC were asked to participate. Doctors participating in these practices were unaware of the syndrome of ACNES. These selection criteria prevented bias and enhanced external validity prior to the start of this screening project.

For proper patient selection, participating practices were required to use an electronic patient record and to register according to the 'International Classification of Primary Care'-codes (ICPC-codes). This system is internationally accepted as standard for coding and classifying complaints, symptoms and diseases in primary care practice. The ICPC system consists of numbered chapters and sections. Chapter D focuses on the digestive tract. It contains 58 codes ranging from malignant disease to functional disorders ${ }^{18}$. The eight codes associated with functional complaints demonstrate a spectrum of various complaints, but only three ICPC-codes focus on complexes of symptoms ('generalized abdominal pain/cramps' (D01), 'other localized abdominal pain' (D06) and 'irritable bowel syndrome' (D93)). For the purpose of this study and the assumption of IBS as a diagnosis of exclusion, these latter three codes were used for patient selection. In absence of a specific ICPC-code for CAWP syndromes, we would advise primary care physicians to use D06 for this clinical entity.

Figure 2 The trigger point is localized and palpated by a palpating finger or a swab. The tenderness increases by abdominal muscle tensing using Carnett's test.

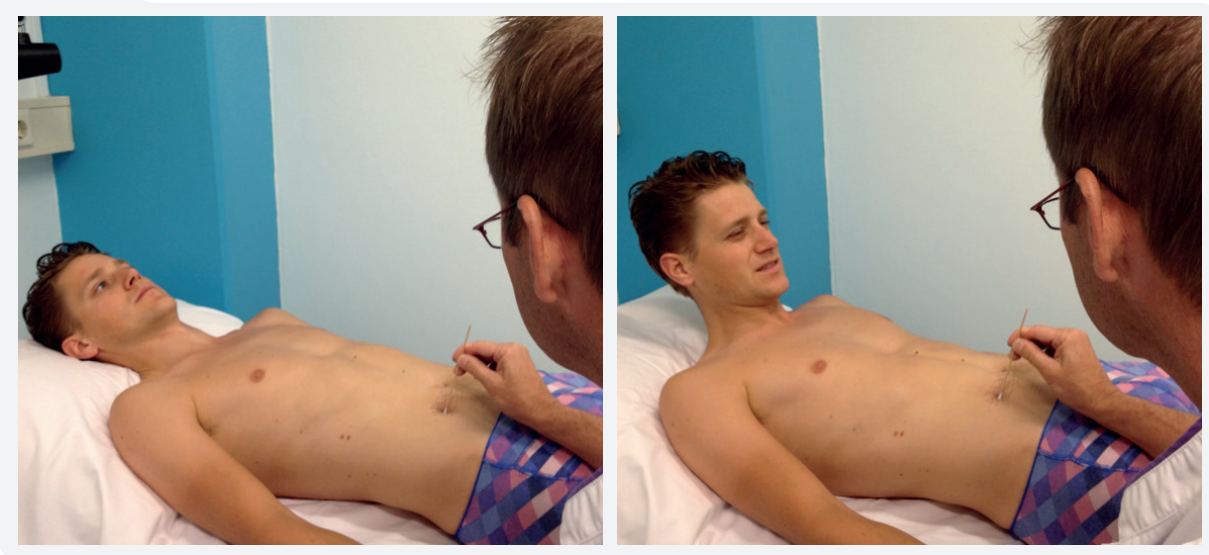


Patients $\geq 18$ years who were registered with ICPC-code D01, D06 and D93 in previous years in these 4 selected practices were eligible for study. No limitations were applied regarding the moment of registration because of the chronic character of the abdominal complaints. Charts of patients with severe comorbidity causing immobility or having additional other gastrointestinal disease were identified manually and excluded as were patients with a language barrier. Remaining subjects were approached by their respective primary care physician via a letter of introduction. Subjects willing to participate were requested to complete the attached 18-pAQ. Non-symptomatic subjects and subjects reporting other reasons not to participate were requested to return the non-participation form. This non-participation form contained two options that could be selected; 'no complaints anymore' and 'other, namely.... Responses were registered and scored after 1 month. Returned and completed questionnaires with more than one missing item were excluded from evaluation.

Due to considerations of logistic nature and feasibility, subjects with a $<10$ point score were not invited for further examination, possibly leading to a slight underestimation of the actual prevalence. Subjects with a $\geq 10$ point score in the $18-\mathrm{pAQ}$ were invited for a standardized history taking and physical examination by the first author. Currently, no golden standard is available for ACNES (and other CAWP syndromes). An abdominal wall syndrome is essentially a clinical diagnosis that is suspected on the basis of a triad of chronic localized abdominal pain within the lateral boundaries of the rectus abdominis muscle (figure 1), a constant site of abdominal tenderness with a small (less than $2 \mathrm{~cm}^{2}$ ) area of maximal intensity (trigger point) and absence of abnormalities in previously taken blood analyses and imaging techniques (colonoscopy, US or CT) if available. Carnett's test is a sensitive clinical test discriminating abdominal wall pain from visceral pain during a physical examination is the Carnett's test (figure 2) 7 $^{7}$ The likelihood of ACNES as a typical example of CAWP syndrome is increased when qualitative local somatosensory disturbances are present in proximity to the trigger point ${ }^{19,20}$. All findings were registered on a case report form.

Data analysis was performed using SPSS 18.0 for Mac OS X. Prevalence numbers were based on symptomatic responders and presented as percentages with associated standard deviations $( \pm \mathrm{SD})$
Figure 3 The validated questionnaire with its answer key. A 10 point cut off value results in an optimal $94 \%$ sensitivity and $92 \%$ specificity

\section{8 item ACNES Questionnaire (18-pAQ)}

\begin{tabular}{|c|c|c|c|c|}
\hline \multicolumn{2}{|c|}{ Question } & \multirow{2}{*}{$\begin{array}{l}\begin{array}{l}\text { Mark the right } \\
\text { answer }\end{array} \\
\text { Mostly / Regularly / } \\
\text { Sometimes / Never }\end{array}$} & \multirow{2}{*}{\begin{tabular}{|l}
1 Point if: \\
Sometimes / Never
\end{tabular}} & \\
\hline 1 & $\begin{array}{l}\text { I experience bloating or a feeling of gas in } \\
\text { the intestines }\end{array}$ & & & \\
\hline 2 & $\begin{array}{l}\text { Pain exists on different spots all over the } \\
\text { abdomen }\end{array}$ & Yes / No & No & \\
\hline 3 & For me PAIN dominates over DISCOMFORT & Yes / No & Yes & \\
\hline 4 & $\begin{array}{l}\text { Lying on the affected side aggravates the } \\
\text { pain }\end{array}$ & $\begin{array}{l}\text { Mostly / Regularly / } \\
\text { Sometimes / Never }\end{array}$ & Mostly / Regularly & \\
\hline 5 & $\begin{array}{l}\text { The stool has an abnormal consistency (e.g. } \\
\text { hard and small, pencil thin, loose, watery) }\end{array}$ & $\begin{array}{l}\text { Mostly / Regularly / } \\
\text { Sometimes / Never }\end{array}$ & Sometimes / Never & \\
\hline 6 & $\begin{array}{l}\text { I believe the pain is originated just beneath } \\
\text { the skin }\end{array}$ & Yes / No & Yes & \\
\hline 7 & Pain is sharp (cutting) & $\begin{array}{l}\text { Mostly / Regularly / } \\
\text { Sometimes / Never }\end{array}$ & Mostly / Regularly & \\
\hline 8 & $\begin{array}{l}\text { I believe the complaints originate from my } \\
\text { gastrointestinal tract }\end{array}$ & Yes / No & No & \\
\hline 9 & $\begin{array}{l}\text { I feel urgent need for bowel movement wit- } \\
\text { hout producing stool (incomplete defecation) }\end{array}$ & $\begin{array}{l}\text { Mostly / Regularly / } \\
\text { Sometimes / Never }\end{array}$ & Sometimes / Never & \\
\hline 10 & $\begin{array}{l}\text { Coughing, sneezing of squeezing aggrava- } \\
\text { tes the pain }\end{array}$ & $\begin{array}{l}\text { Mostly / Regularly / } \\
\text { Sometimes / Never }\end{array}$ & Mostly / Regularly & \\
\hline 11 & Pain is always located on the same spot(s) & Yes / No & Yes & \\
\hline 12 & $\begin{array}{l}\text { Pain is located just lateral to the midline of } \\
\text { the abdomen }\end{array}$ & Yes / No & Yes & \\
\hline 13 & $\begin{array}{l}\text { Complaints are somehow related to an alte- } \\
\text { red defecation pattern }\end{array}$ & Yes / No & No & \\
\hline 14 & $\begin{array}{l}\text { Pain is provoked by daily activities (e.g. } \\
\text { walking, sitting, cycling, bending) }\end{array}$ & $\begin{array}{l}\text { Mostly / Regularly / } \\
\text { Sometimes / Never }\end{array}$ & Mostly / Regularly & \\
\hline 15 & $\begin{array}{l}\text { The painful spot feels strange, different or } \\
\text { dull }\end{array}$ & $\begin{array}{l}\text { Mostly / Regularly / } \\
\text { Sometimes / Never }\end{array}$ & Mostly / Regularly & \\
\hline 16 & Stress provokes the complaints & $\begin{array}{l}\text { Mostly / Regularly / } \\
\text { Sometimes / Never }\end{array}$ & Sometimes / Never & \\
\hline 17 & $\begin{array}{l}\text { The most intense pain can be localized by } \\
\text { the tip of one finger }\end{array}$ & Yes / No & Yes & \\
\hline 18 & $\begin{array}{l}\text { Pushing on the painful spot aggravate the } \\
\text { complaints }\end{array}$ & $\begin{array}{l}\text { Mostly / Regularly / } \\
\text { Sometimes / Never }\end{array}$ & Mostly / Regularly & \\
\hline & & & & \\
\hline
\end{tabular}




\section{Results}

A total of 583 patients were identified as dictated by the 3 ICPC-codes of interest in the 4 selected primary care practices. After exclusion based on age $<18$ years $(n=6)$, comorbidity $(n=13)$, other known gastrointestinal disease $(n=8)$, absence of complaints $(\mathrm{n}=2)$ and personal reasons $(\mathrm{n}=19), 535$ patients were eligible for screening. Some 369 patients were registered with ICPC code D93 (irritable bowel syndrome). The remaining subjects $(n=166)$ were diagnosed with generalized or localized abdominal pain (D01, D06).

The response rate was $57 \%(n=304)$. Of this responding population, questionnaires of 167 subjects signing informed consent were analysed (female $68 \%$, age $54 \pm 17$ years). The remaining 137 subjects did not participate in screening (no complaints at present, $n=94$; personal reason, $\mathrm{n}=37$; missing items in the $18-\mathrm{pAQ}, \mathrm{n}=6$ ).

Twenty-three of these 167 patients scored 10 points or more on the 18-pAQ. After being contacted, 18 individuals were willing to undergo history taking and physical examination. Nine subjects were found to have IBS, generalized abdominal pain/cramps, or other localized abdominal pain as diagnosed previously. The other half of this group $(\mathrm{n}=9)$ was diagnosed with a CAWP syndrome. Six of these were having ACNES (D93, $\mathrm{n}=4 ; \mathrm{D} 01, \mathrm{n}=2$ ). The remaining three patients were diagnosed with a painful lipoma $(\mathrm{n}=1)$, an abdominal wall herniation $(\mathrm{n}=1)$ and scar tissue pain $(\mathrm{n}=1)$ on the basis of physical examination and ultrasound investigation (figure 4).

Of the six identified ACNES patients, four received a subfascial trigger point injection using $1 \%$ of lidocain with long lasting satisfactory pain reduction (> 3 months) in three. The fourth ACNES patient received additional manual therapy as the pain was refractory to injections. A neurectomy is considered if she remains unresponsive ${ }^{14,20}$. Pain levels of patient five and six were mild. These two individuals were reassured once the origin of pain was explained. Despite the somewhat smaller sample size then calculated in the power analysis, a prevalence of ACNES was calculated to be 3.6\% (95\%CI 1.7-7.6) within the symptomatic functional abdominal pain population in these 4 selected primary care practices.
Figure 4 Flow chart of the study protocol

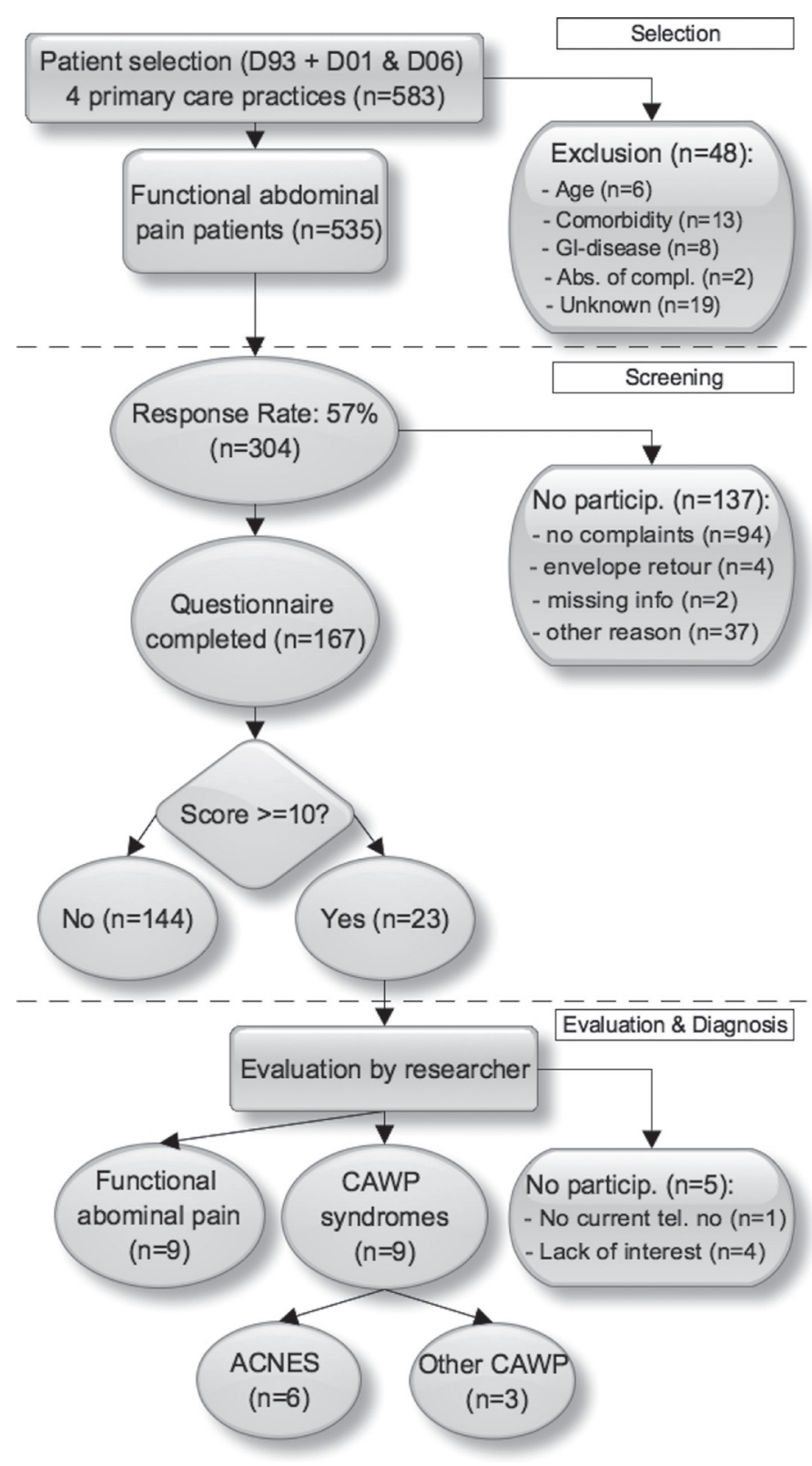




\section{Discussion}

The present study demonstrates that ACNES may be diagnosed in some patients currently treated for or diagnosed with functional abdominal complaints such as IBS. Six subjects harboured characteristics that are typically associated with ACNES, corresponding to a $3.6 \%$ prevalence $(95 \%$ CI $1.7-7.6)$ in the responding 'alleged' IBS population of 167 individuals. This percentage is based on symptomatic responders. Although this may seem a relatively small number, a substantial portion of the Dutch population is registered as having functional abdominal complaints. Estimations on the prevalence of IBS in the Netherlands may vary from 10-20\% (population 16.7 million individuals ${ }^{21}$ [Statistiek, \#174][Statistiek, \#174]). Considering that 1 of 3 persons having complaints related to IBS seeks medical help, a minimum of half a million individuals is calculated to consult a doctor for this problem in the Netherlands $s^{22-25}$. Based on these assumptions, some 20 thousand Dutch individuals with ACNES are currently misdiagnosed as having functional abdominal pain (95\%CI 9.500 - 42.000). Using similar assumptions and calculations, some 400.000 US individuals with functional abdominal syndromes may suffer from an undiagnosed CAWP such as ACNES ${ }^{26,27}$.

During the study's analysis period, a seventh ACNES patient was identified by one of the participating primary care physicians, now familiarized with the typical diagnostic triad of ACNES. Remarkably, this patient was registered with an ICPC-code belonging to the 'nervous system' chapter, probably due to predominant somatosensory alterations of the abdominal skin. This newly diagnosed CAWP patient illustrates the diversity of interpretations by primary care physicians who are unaware of ACNES. However, an important observation of the present study is the immediate effect of heightened awareness amongst primary care physicians regarding a CAWP syndrome.

A survey among Dutch surgeons and residents confirmed a hypothesized ignorance of ACNES among hospital based specialists. More than $85 \%$ of the respondents confessed they had never heard of this syndrome (response rate 35\%,507/1450). Moreover, only $18 \%$ of senior surgeons (>10 years of experience) were aware of this clinical entity ${ }^{28}$. It is not surprising that doctors' delay in diagnosing CAWP syndromes is substantial and many patients with ACNES remain undiagnosed. This situation is not typical Dutch as CAWP is a globally undervalued medical problem and a diagnostic challenge. Therefore, clinicians worldwide require education on abdominal wall pain problems so that these clinical entities will be identified in an earlier stage and treated accordingly.
A complicating factor is the fact that ACNES patients often report 'pseudo-visceral' complaints (bloating, nausea or pain related to e.g. food intake, altered defecation pattern, weight loss) seemingly unrelated to an abdominal wall problem ${ }^{4,5}$.

The present diagnostic questionnaire was found to harbour good discriminative properties during the validation study ${ }^{17}$. In a low prevalence population as the current one, a $50 \%$ positive predicted value is suboptimal but acceptable. As a comparison, the positive predictive value of a national screening program for breast cancer in the Netherlands is $41 \%^{29}$. However, one can question if this tool is performing appropriately in the hands of physicians with little knowledge on CAWP syndromes. Considering the suboptimal sensitivity of the used questionnaire, it is likely that some CAWP patients remained unidentified in the investigated cohort. However, a subpopulation may be identified (approximately 10-15\% of the total population) by the questionnaire and invited for specific examination.

The present study has shortcomings. A flaw is the relatively low response rate of subjects completing the $18-\mathrm{pAQ}$ resulting in a prevalence number with a wide confidence interval. One explanation is that approximately a third of all responders (31\%) claimed to have no complaints at present and therefore did not complete the questionnaire. Moreover, the largest group of non-participators did not respond at all (43\%). Helpseeking patients probably are more likely to respond. Furthermore, all patients who were ever diagnosed with IBS or localized/generalized abdominal pain were eligible for study. This inclusion criterion was deemed important as symptoms intensity of both IBS and ACNES may fluctuate over time. A low response rate in patients having few symptoms at the time of questionnaire reception is therefore likely. Another point of concern is a possible flaw in the ICPC-code registration process. Under-registration and updating of patients' files may be suboptimal in a demanding practice.

What is the clinical relevance of identifying patients with ACNES in a group in which the majority of patients have IBS? On the one hand, patients diagnosed with ACNES (preferably in an early stage by their physician) are likely to undergo fewer unnecessary medical procedures and may benefit from a relatively simple treatment regimen. A treatment stratagem including injections or surgery resulted in total or substantial long-term pain relief in $80 \%$ of patients with $\mathrm{ACNES}^{14}$. On the other hand, even after an unsuccessful IBS treatment an alternative diagnosis is not easily considered. This is especially due to the low treatment effectiveness of IBS (less then $60 \%$ benefits from treatment using antispasmodics and antidepressant ${ }^{30}$ ). Wrongly diagnosed patients 
with abdominal wall pain most probably do not have any effect of these IBS treatment strategies. Moreover, a proper and effective treatment regimen is not instituted leading to prolonged suffering.

\section{Conclusion}

A clinically relevant portion of patients wrongfully diagnosed with a functional abdominal pain syndrome within the primary care setting is found to suffer from a chronic abdominal wall pain (CAWP) syndrome including the anterior cutaneous nerve entrapment syndrome (ACNES). Once correctly diagnosed, treatment is simple and effective. Increased awareness of CAWP syndromes may have a considerable effect on an individual level as well as on the level of the health care system.

\section{References}

1. WGO Practice Guideline. Irritable bowel syndrome, a global perspective. Milwaukee, WI: World Gastroenterology Organisation, 2009. Available from: http:// www worldgastroenterology.org/irritable-bowelsyndrome.html. Accessed September 9, 2013.

2. Spiegel BM, Farid M, Esrailian E, et al. Is irritable bowel syndrome a diagnosis of exclusion?: a survey of primary care providers, gastroenterologists, and IBS experts. Am J Gastroenterol. 2010; 105: 848-858.

3. Sharpstone D, Colin-Jones DG. Chronic, non-visceral abdominal pain. Gut. 1994; 35: 833-836.

4. Choi YK, Chou S. Rectus syndrome. Another cause of upper abdominal pain. Reg Anesth 1995; 20: 347-351.

5. Haynsworth RF, Jr., Noe CE. An unusual presentation of intercostal neuralgia. Anesthesiol. 1990; 73: 779-780.

6. Srinivasan R, Greenbaum DS. Chronic abdominal wall pain: a frequently overlooked problem. Practical approach to diagnosis and management. Am J Gastroenterol. 2002; 97: 824-830.

7. Carnett J. Intercostal neuralgia as a cause of abdominal pain and tenderness. Surg Gynecol Obstet. 1926; 42: 8.

8. Applegate WV. Abdominal cutaneous nerve entrapment syndrome. Surgery. 1972; 71: $118-124$

9. Hershfield NB. The abdominal wall. A frequently overlooked source of abdominal pain. J Clin Gastroenterol. 1992; 14: 199-202.

10. Greenbaum DS, Greenbaum RB, Joseph JG, et al. Chronic abdominal wall pain. Diagnostic validity and costs. Dig Dis Sci. 1994; 39: 1935-1941.

11. Abdominal wall tenderness test: could Carnett cut costs? Lancet. 1991; 337: 1134.

12. Suleiman S, Johnston DE. The abdominal wall: an overlooked source of pain Am Fam Physician. 2001; 64: 431-438.

13. Applegate WV. Abdominal Cutaneous Nerve Entrapment Syndrome (ACNES) A Commonly Overlooked Cause of Abdominal Pain. 2002; 6.

14. Boelens OB, Scheltinga MR, Houterman S, et al. Management of anterior cutaneous nerve entrapment syndrome in a cohort of 139 patients. Ann Surg. 2011; 254: 1054-1058.

15. Rome Foundation [homepage]. Available from: http://www.romecriteria.org/. Accessed September 9, 2013. 
16. Naing L. Practical Issues in Calculating the Sample Size for Prevalence Studies. Arch Orofacial Sc. 2006; 1: 9-14.

17. van Assen T, Boelens OB, Kamphuis JT, et al. Construction and validation of a questionnaire distinguishing a chronic abdominal wall pain syndrome from irritable bowel syndrome. Frontline gastroenterol. 2012; 3: 288-294.

18. Njoo K. ICPC. 2012. Available from: https://www.nhg.org/themas/artikelen/icpc. Accessed November 1, 2009.

19. Boelens OB, Scheltinga MR, Houterman S, et al. Randomized clinical trial of trigger point infiltration with lidocaine to diagnose anterior cutaneous nerve entrapment syndrome. $\mathrm{Br}$ J Surg. 2013; 100: 217-221.

20. Boelens OB, van Assen T, Scheltinga MR, et al. A double blind randomized controlled trial on surgery for chronic abdominal pain due to anterior cutaneous nerve entrapment syndrome. Ann Surg. 2013;257:845-9.

21. Statistiek CBvd. Bevolkingsteller. [web page] [cited 2012; Available from: http://www.cbs. nl/nl-nl/menu/themas/bevolking/cijfers/extra/bevolkingsteller.htm.

22. Drossman DA, Whitehead WE, Camilleri M. Irritable bowel syndrome: a technical review for practice guideline development. Gastroenterol. 1997; 112: 2120-2137.

23. Talley NJ, Weaver AL, Zinsmeister AR, et al. Onset and disappearance of gastrointestinal symptoms and functional gastrointestinal disorders. Am J Epidemiol. 1992; 136: 165-177.

24. Van der Velden J. [A national study for diseases and procedures in the primary care practice. Base report; Morbidity in the primary care practice]. NIVEL, 1991.

25. Okkes I. Oskam SK, Lamberts H. [From complaint to diagnosis], Bussum: Coutinho; 1998.

26. Saito YA, Schoenfeld P, Locke GR. The epidemiology of irritable bowel syndrome in North America: a systematic review. Am J Gastroenterol. 2002; 97: 1910-1915.

27. U.S. and world population clock. Washington, DC: US Census Bureau. Available from: http://www.census.gov/main/www/popclock.html. Accessed September 9, 2013.

28. Roumen RM, Scheltinga MR. [Abdominal intercostal neuralgia: a forgotten cause of abdominal pain]. Ned Tijdschr Geneeskd. 2006; 150: 1909-1915.

29. CBO, K.v.d.g., [Guideline Breast cancer, N.B.O. Netherlands, Editor 2008, Quality institute for health care $\mathrm{CBO}$ ]: Utrecht. p. 274.

30. Ruepert L, Quartero AO, de Wit NJ, et al. Bulking agents, antispasmodics and antidepressants for the treatment of irritable bowel syndrome. Cochrane Database Syst Rev. 2011: CD003460. 


\section{Chapter 4}

Incidence of abdominal pain due to the anterior cutaneous nerve entrapment syndrome in an emergency department

Tijmen van Assen

Jill A.G.M. Brouns

Marc R. Scheltinga

Rudi M. Roumen 


\section{Abstract}

Background

Patients with chronic abdominal pain occasionally suffer from the anterior cutaneous nerve entrapment syndrome (ACNES). A substantial number of patients report previous visits to an emergency department (ED) with acute pain. Aim of this study was to obtain the incidence of ACNES in patients presenting with abdominal pain in the ED of a Dutch teaching hospital.

Methods

In this observational study, data sets of all patients visiting Máxima Medical Center's (MMC) ED in 2011-2012 were searched for key terms 'ACNES', 'intercostal neuralgia' or 'abdominal wall pain'. Files of potential patients living within hospital's adherence area were checked using accepted criteria indicating the presence of ACNES. Disease incidence was calculated as a ratio to the hospital's adherence data. The ACNES MMC 2013's incidence in patients evaluated in the surgical outpatient department was also calculated.

\section{Results}

During the study period, $473 \mathrm{ED}$ patient files met inclusion criteria. A total of 88 patients belonging to MMC's adherence area were diagnosed with ACNES leading to a mean 22/100.000 ACNES yearly incidence rate. About one of 50 patients with abdominal pain visiting the ED suffered from ACNES. A 35/100.000 outpatient department ACNES incidence rate was calculated in the year of 2013. Combining these two ratios, a 1:1800 ACNES incidence in the general population was obtained.

\section{Conclusions}

In an ED setting of a Dutch teaching hospital, approximately $2 \%$ of patients presenting with acute abdominal pain suffered from ACNES. ED physicians should consider ACNES in abdominal pain patients with normal laboratory or imaging tests.

\section{Background}

Patients with abdominal pain may suffer from an abdominal wall related entity. A typical example is the anterior cutaneous nerve entrapment syndrome (ACNES) ${ }^{1-9}$. End branches of thoracic 8-12 intercostal nerves are caught in abdominal muscles causing severe neuropathic pain ${ }^{2,3,6,10,11}$. If recognized, injecting anaesthetic agents into the point of maximal pain is diagnostic and occasionally therapeutic ${ }^{12,13}$. If pain persists, removal of nerve end twigs may offer relief ${ }^{12,14-17}$.

A literature survey on incidence of acute abdominal pain in a general emergency department (ED) demonstrated that approximately $1 \%$ of referrals to general surgeons were related to these acute abdominal wall pain syndromes ${ }^{9}$. Moreover, $20 \%$ of patients who presented to the ED with a nonspecific acute abdominal pain tested positive for abdominal wall tenderness suggesting the presence of an abdominal wall pain syndrome ${ }^{18}$. However, such estimations are biased by author selection and preference. Therefore, a reliable estimation of incidence of acute abdominal wall pain syndromes such as acute ACNES presenting to an ED is not available.

Physicians working in the SolviMáx centre for abdominal wall and groin pain syndromes are experienced in diagnosing ACNES in adults and adolescents $\mathrm{s}^{6}, 11-17,19$. During outpatient department intakes, these ACNES patients frequently reported on earlier ED visits with attacks of abdominal pain. Unfortunately, pain was then often not recognized as due to nerve entrapment leading to a substantial doctor's delay ${ }^{2,7,20}$. With this finding in mind, awareness in an ED regarding ACNES may seem suboptimal. However, if a triad of abdominal pain, a circumscript pain point (in the presence of a positive Carnett test and/or local sensory disturbances) and a normal blood analysis or ultrasonography/computed tomography are present, a person may suffer from this typical 'clinical syndrome'.

Aim of the present study was to calculate the incidence of ACNES in the ED of a Dutch teaching hospital. If substantial, ACNES must attain a more prominent position on the list of differential diagnoses of acute abdominal pain in an ED. 


\section{Methods}

General information

Data of this retrospective observational study was obtained between 2011 and 2014 in Máxima Medical Centre (MMC), a 543-bed community hospital situated in the southern part of The Netherlands serving a population of approximately 200.000 inhabitants. MMC is considered a typical example of an average Dutch teaching hospital. Approximately 30 thousand patients present to its ED each year. Comparable to published numbers, some 8 percent of visits to MMC's emergency department is due to abdominal pain ${ }^{21-23}$. Each abdominal pain patient is standardly evaluated by interns of the ED, surgery or internal medicine. If deemed necessary, patients are subsequently evaluated by a senior emergency physician or by a consultant (emergency medicine, surgery, internal medicine, gastroenterology, urology etc.).

A surgical sub department - separate from the ED - has gained ample experience in the evaluation of patients with chronic abdominal wall pain and groin pain syndromes ('SolviMáx' Center of excellence for Abdominal Wall and Groin Pain) '13, 14, 24. MMC physicians are regularly updated on new evidence regarding ACNES by these SolviMáx specialists. Therefore, awareness on our ED regarding ACNES is likely higher than ED's of other (Dutch) hospitals.

The MMC Medical Ethical Committee concluded that the rules laid down in Medical Research Involving Human Subjects Act did not apply to the present research proposal and hereby gave permission to perform the study (N15-004).

Data collection

Electronically stored data using the ChipSoft $\odot$ health and information system (CS-EHIS, version 5.2 HF188, ChipSoft B.V., The Netherlands) served as the source for the present study. This software program is used by all MMC health care providers for documentation of patient visits25. Two sets of data were extracted from this CS-EHIS system. The first set contained data of all patients who visited the MMC's ED in the year of 2011 and 2012. The second dataset consisted of surgical patient records containing one of the following keywords: 'ACNES', 'intercostal neuralgia' or 'abdominal wall pain'. By electronically combining these two data sets, patients of interest were identified and were inserted in a separate data file termed 'potential ACNES-ED patient file (ACNES-EDPF)' that was used as the basis for the present study.
As ACNES is generally considered a typical clinical syndrome, a diagnostic gold standard is currently lacking. However, the diagnosis was deemed likely in a patient if the following set of signs and symptoms, as registered in CS-EHIS, were met:

1. Presenting with abdominal pain at the ED, and

2. Site of abdominal tenderness with a small ( $<2 \mathrm{~cm} 2$, "finger tip") area of maximal intensity (trigger point) situated within lateral boundaries of the rectus abdominis muscle, and

3. Normal laboratory analysis and normal imaging (if performed for this specific ED visit), and

4. Pain reduction following infiltration using $5-10 \mathrm{~mL}$ of $1 \%$ lidocaine, and

5. The diagnosis ACNES was confirmed during a follow up ED visit, or shortly thereafter during a visit to one of MMC's surgical outpatient departments by one of the SolviMáx pain specialists as reflected in the surgical patient record.

If the diagnosis in an individual that was present in this ACNES-EDPF file was deemed uncertain, history and physical examination, as well as diagnostic and therapeutic measures that were tabulated in the patients' file were again checked for additional specifics and clues. If still in doubt, one of the SolviMáx pain specialists $(n=4)$ was consulted for advice. Typical findings such as a positive Carnett's test, a positive pinch test and the presence of local sensory disturbances or lateral/paravertebral trigger points strongly contributed to confirmation of the diagnosis (figure 1. a-d) ${ }^{2,3}$.

Known ACNES patients who visited the ED were excluded. Patients who were suspected of having ACNES whereas history and findings at physical examination were not consistent with the syndrome were also excluded. These patients were usually referred to the ED by family physicians. To correct for spectrum bias, individuals living outside MMC's adherence area were excluded as well.

Accurate figures on MMC adherence were obtained from a market analysis. In the year of 2011, a total of 206.000 individuals were assumed to visit the MMC for medical assistance. In the year of 2012, this adherence number was officially set at 199.000 residents (Kiwa Carity B.V., Utrecht, The Netherlands). These numbers are considered to be an optimal approximation of MMC's real adherence.

An additional calculation of the 2013 incidence of chronic ACNES was based on first time patient visits to the SolviMáx outpatient department. Each first SolviMáx outpatient department visit of an individual referred as possibly having ACNES is prospectively 
registered from January 1, 2013. Electronic patient files of all individuals diagnosed with ACNES via this system were also studied. Patients were excluded if they lived outside of MMC's adherence area, if they were known ACNES patients, or if they had presented to MMC's ED in an earlier stage (2013 MMC adherence, approximately 200.000).

Figure 1 Typical findings during physical examination in an ACNES patient.

a. Trigger point is localized using palpating index finger. Tenderness increases when abdominal muscles are tensed as the patient lifts the head (Carnett's test);

b. Skin gnostic sensibility is determined using a swab. The shaded area overlying the trigger point reflects altered sensibility;

c. A cold alcohol gauze is utilized to test the vital sensibility;

d. By squeezing a fold containing the patient's skin and subcutaneous fat between ('pinch test')

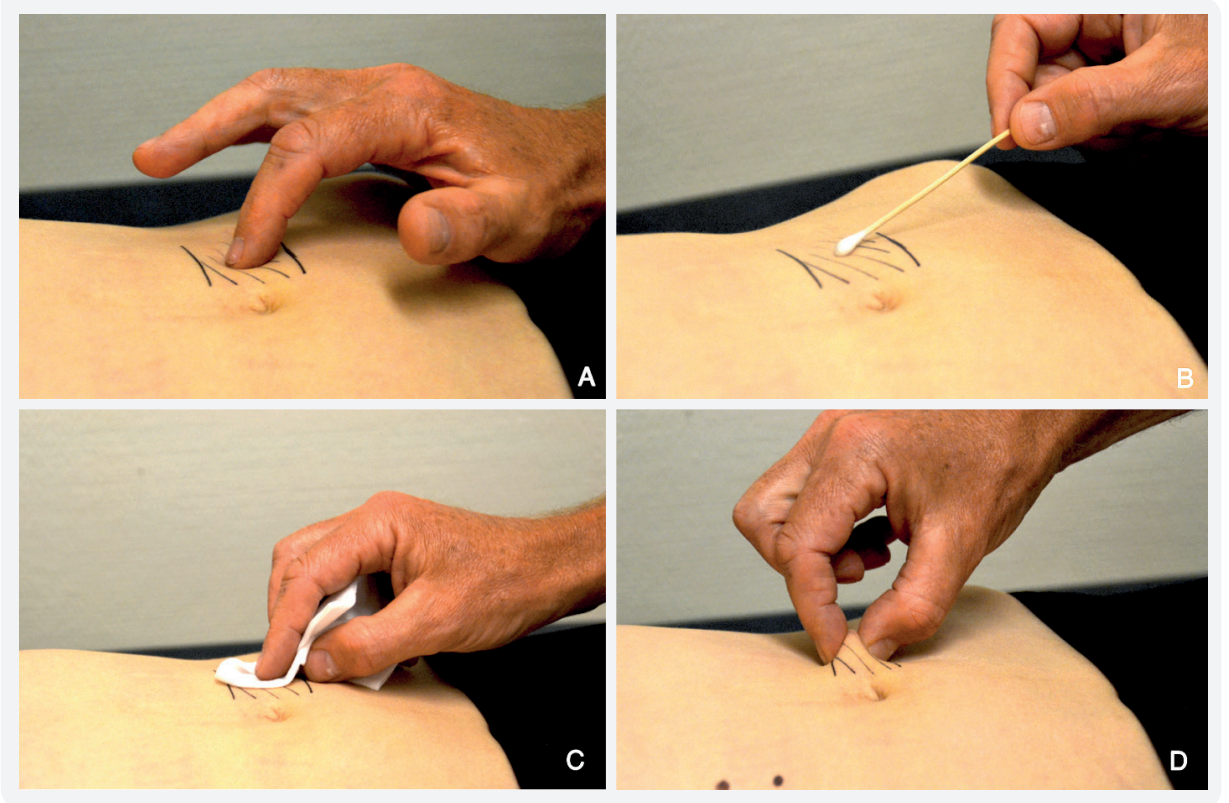

Data analysis

Data analysis was performed using SPSS 21.0 for Mac OS X (IBM SPSS statistics, IBM corporation, New York, United States). Baseline characteristics were presented as percentages, mean (SD), or median values (range) as appropriate. Adherence numbers of MMC and numbers of ACNES patients diagnosed in 2011 and 2012 were used to calculate an ACNES ED incidence and a mean incidence rate using standard calculus.

\section{Results}

Incidence of ACNES on ED in 2011 and 2012

In the year of 2011, 31.325 patients attended the ED of MMC (2012: 30.023). In the 2-year study period, 5.111 patient visits were registered for abdominal pain $(8.3 \%, 5111 / 61.348)$ A total of $473 \mathrm{ED}$ patient files harboured the preselected keywords 'ACNES', 'intercostal neuralgia' or 'abdominal wall pain'. A total of 186 patients (39\%, table 1) was also identified via these keywords. However, they were excluded as they visited the ED for a non-abdominal illness $(\mathrm{n}=186)$

In 2011, 41 ED patients with (acute) abdominal pain were diagnosed with ACNES, the majority ( $\mathrm{n}=35)$ belonging to MMC adherence area (table 1, left hand panel). As 206.000 registered individuals adhered to the MMC area, a 17/100.000 incidence rate was calculated. A similar calculation regarding the year of 2012 (56 ACNES, 53 in a 199.000 MMC adherence) yielded a 27/100.000 incidence rate. As a consequence, a mean 22/100.000 ACNES ED incidence rate per year was obtained over these two years. If al 5.111 patient visits with abdominal entities in the ED were considered, approximately 1:50 was found to have ACNES.

Table 1 Patients potentially having ACNES visiting the emergency department of a Dutch teaching hospital in 2011-2012

\begin{tabular}{lccc} 
& $\mathbf{2 0 1 1}$ & $\mathbf{2 0 1 2}$ & $\mathbf{2 0 1 1 / 2 0 1 2}$ \\
\hline $\begin{array}{l}\text { Patient file containing key words 'ACNES', 'intercostal neuralgia' or } \\
\text { 'abdominal wall pain' ( } \mathrm{n} \text { ) }\end{array}$ & 250 & 223 & 473 \\
Presenting to ED with a non abdominal illness (n) & & & \\
Trauma & 114 & 72 & 186 \\
Fever / malaise & 52 & 27 & 79 \\
Pulmonary & 19 & 6 & 25 \\
Cardiac & 10 & 5 & 15 \\
$\quad$ Neurological & 9 & 11 & 20 \\
Skin infection and/or abscess & 2 & 3 & 5 \\
Collapse & 7 & 5 & 12 \\
Other & 1 & 3 & 4 \\
Presenting with abdominal pain (n) & 14 & 12 & 26 \\
ACNES (n) & 136 & 151 & 287 \\
Excluding patients outside MMC adherence area (n) & 41 & 56 & 97 \\
Diagnosis ACNES within MMC adherence area (n) & -6 & -3 & -9 \\
\hline
\end{tabular}


Baseline characteristics, history and findings of physical examination in this population of 88 ACNES patients are summarized in table 2 . The majority was female $(\mathrm{n}=71,81 \%)$, and median age was 37 years (range 12-76). Most $(\mathrm{n}=62,70 \%)$ patients reported that the pain had started in the previous week. A typical right lower abdominal preponderance $(59 \%, \mathrm{n}=52)$ was found.

Table 2 Characteristics of patients diagnosed with ACNES ( $n=88)$ in a Dutch emergency department during a two year period (2011/2012)

\begin{tabular}{lc}
\hline Age, m2dian (range) & $37(12-76)$ \\
Gender (M:F) & $17: 71$ \\
Duration of symptoms & 62 \\
$<1$ week & 14 \\
1 week - 1 month & 10 \\
1 month -6 months & 2 \\
$>6$ months & \\
Physical examination & 13 \\
Abdominal pain location, $n$ & 5 \\
$\quad$ Right upper quadrant & 52 \\
Left upper & 17 \\
Right lower & 1 \\
Left lower & \\
Bilateral lower & $88: 0(0)$ \\
Trigger point, Yes : No (missing data, n) & $65: 5(18)$ \\
Carnett-sign, Pos : Neg (missing) & $60: 9(19)$ \\
Sensory disturbances, Y : N (missing) & $37: 5(46)$ \\
\hline Pinch, Pos : Neg (missing) &
\end{tabular}

\section{3 outpatient department ACNES incidence}

In the year of 2013, a total of 268 new onset patients visiting the SolviMáx outpatient department were diagnosed with ACNES. However, just a small portion $(85 / 268,32 \%)$ belonged to the adherence area of MMC confirming its status as a referral centre for the remaining $68 \%$. As 15 of these 85 newly diagnosed ACNES patients had attended the ED of MMC in an earlier phase, a 35/100.000 incidence rate of ACNES in the outpatient department was calculated. By combining numbers of ED and outpatient department, a total 57/100.000 ACNES incidence rate was obtained.

\section{Discussion}

It is our experience that some patients who are ultimately diagnosed with chronic abdominal pain due to the anterior cutaneous nerve entrapment syndrome (ACNES) reported that they had previously attended emergency departments (ED) for earlier attacks of pain. As blood analysis, ultrasounds or CT-scans were always inconclusive, they were frequently sent home without receiving a conclusive diagnosis resulting in a substantial doctor's delay. To this day, ACNES is consistently neglected as a cause of abdominal pain $^{2,5,7,8}$. Awareness regarding ACNES as part of the differential diagnosis of chronic abdominal pain in our area is high ${ }^{6,11-17,19}$. It may be assumed that the chances of recognizing ACNES in patients with acute abdominal pain visiting our ED are also higher. Aim of the present study was to calculate the incidence of ACNES in the ED of a Dutch teaching hospital. Based on an analysis of all patients visiting during the 2011/2012 time period, a mean 22/100.000 per year incidence rate was obtained. In perspective, about one of 50 patients presenting with abdominal pain to the ED was diagnosed to suffer from ACNES. In an ED such as MMC's hosting approximately 30 thousand patients yearly, the diagnosis ACNES is made almost once a week. An emergency physician must realize that a patient who is referred to the $\mathrm{ED}$ for abdominal pain of unknown origin may suffer from an abdominal wall pain syndrome such as ACNES.

Most patients with chronic abdominal pain due to ACNES are diagnosed after a substantial time period. For instance, mean doctor's delay was more than a year in one study indicating that the pain is per definition chronic ${ }^{13}$. Interestingly, most patients in the current study (70\%) presented to the ED within the first week following the onset of pain. Although generally considered a chronic entity, a substantial portion of ACNES patients apparently experiences pain in an acute setting. This novel finding clearly illustrates our limited knowledge on various issues regarding the syndrome. As a consequence, most patients with an acute ACNES syndrome probably move on to develop a chronic form due to insufficient awareness of consulted doctors.

How can consciousness regarding signs characterizing ACNES be increased? A physical examination has a pivotal role in the diagnosis. If serious causes of acute abdominal pain are excluded in the ED using a combination of physical examination, blood analysis and imaging techniques, results of simple tests may point towards the presence of ACNES. A positive Carnett's test and an area of sensory disturbances covering the painful spot are signs reflecting the neuropathic character of this pain syndrome $e^{2,7,10}$ $18,26,27$. We routinely evaluate skin gnostic sensibility using a simple swab. Hypoesthesia, 
hyperesthesia/algesia or sometimes even allodynia may be found by comparison to the normal contralateral abdominal side. In addition, a gauze soaked with alcohol is utilized to test vital (cold) sensibility. A positive pinch test is a sensitive and highly underrated sign reflecting the presence of a neuropathic pain syndrome ${ }^{2}$. By squeezing a patient's skin fold and subcutaneous fat using thumb and index finger, the area of interest will hurt when compared to the contralateral side (figure 1). The majority of patients $(88 \%, 37 / 42)$ in the present study tested positive. A skin pinch test deserves a standard place in the ED physician's toolbox of physical examinational tests for abdominal pain.

When the diagnosis ACNES is likely, ED physicians are advised to start trigger point injection therapy. A subfascial injection of 5-10 $\mathrm{ml}$ of 1-2 \% lidocaine leading to immediate pain reduction is diagnostic and occasionally therapeutic. Previous studies in chronic ACNES demonstrated permanent pain relief in one-thirds of the patients after injection therapy ${ }^{12,13}$. Patients with recalcitrant pain after repetitive injections are offered resection of entrapped branches of the intercostal nerve leading to long-term pain remission in up to three-quarters ${ }^{12,16}$. In the remaining population, a renewed surgical exploration is found to be effective in an additional two-thirds of the patients. This three-step treatment protocol may lead to pain remission in some ninety percent ${ }^{17}$. Interestingly, the response to immediate injection therapy may be more favourable in acute ACNES patients. For instance, a conservative strategy sufficed in the majority $(64 / 88,73 \%)$ of the present study population. Conversely, just 24 patients $(27 \%)$ in the study population underwent a neurectomy comparing favourably to a 50\% surgical rate as reported in another group of chronic patients who were still painful after injection therapy ${ }^{12}$. An optimized response to injection therapy in acute ACNES may be related to the short duration of complaints. This hypothesis is currently tested in our patient population with ACNES.

How does the present ACNES incidence relate to other known disease entities in the Netherlands? The calculated 57/100.000 (1:1800) total ACNES incidence is obtained in our area containing approximately 200.000 individuals. If extrapolated to the Dutch population (16.800.000 inhabitants), some 9.500 new ACNES patients may be identified yearly ${ }^{28}$. In a survey among alleged irritable bowel syndrome patients in a general practice setting in the Netherlands, a prevalence of some 20 thousand ACNES patients (being approximately $4 \%$ in relative numbers) within this functional abdominal pain population was calculated ${ }^{11}$. The 9.500 ACNES incidence yearly exceeds numbers of Dutch patients that are newly diagnosed with for instance inflammatory bowel disease $(\mathrm{n}=7.200)$ or stomach and duodenum ulcer $(\mathrm{n}=6.700)^{29}$. The incidence number of ACNES patients visiting the $\mathrm{ED}$ is calculated at 1:4.500 (22:100.000). It is interesting to relate these numbers to for instance the well-known incidence of acute appendicitis (approximately 1:1.000). For every 4-5 acute appendicitis patients, one additional individual with acute abdominal pain is diagnosed with $\mathrm{ACNES}^{30}$. From a different perspective, as an average Dutch general practice contains about 2500 patients, a Dutch general practitioner will be confronted with 1-2 new ACNES patients yearly.

The current study harbours flaws associated with its observational character including incomplete data sets. For instance, some specific characteristics of history taking and physical examination were not routinely registered possibly indicating bias. Moreover, the electronic searching strategy may have been incomplete as the number of used key words in the CS-EHIS may have been insufficient or incorrectly registered by ED physicians. Although these effects are considered minimal, an incidence underestimation rather than an overestimation may have resulted from these flaws. Another disadvantage is potentially related to the institution where this study is executed. Although groups of physicians working on MMC's ED are rotating, there is no doubt that awareness regarding this neglected syndrome is high compared to other ED's. Therefore, there may be a risk of 'overdiagnosing' patients with abdominal wall pain syndromes, also because the diagnosis ACNES is a typical clinical diagnosis lacking a gold standard. However, we strive to strictly adhere to objective criteria. Moreover, no alternative abdominal diagnoses emerged following presentation at the ED or during follow up.

\section{Conclusion}

Approximately $2 \%$ of all patients presenting with acute abdominal pain were diagnosed with anterior cutaneous nerve entrapment syndrome (ACNES) in an emergency department setting. If patients with a localized acute abdominal syndrome demonstrate normal laboratory or imaging testing, ED physicians should consider ACNES in the differential diagnosis. 


\section{References}

1. Applegate WV. Abdominal cutaneous nerve entrapment syndrome. Surgery. 1972; 71: 118-124.

2. Applegate WV. Abdominal Cutaneous Nerve Entrapment Syndrome (ACNES): A Commonly Overlooked Cause of Abdominal Pain. Perm J. 2002; 6: 20-27.

3. Carnett J. Intercostal neuralgia as a cause of abdominal pain and tenderness. Surg Gynecol Obstet. 1926; 42: 8 .

4. Choi YK, Chou S. Rectus syndrome. Another cause of upper abdominal pain. Reg Anesth. 1995; 20: 347-351.

5. Hershfield NB. The abdominal wall. A frequently overlooked source of abdominal pain. J Clin Gastroenterol. 1992; 14: 199-202.

6. Roumen RM, Scheltinga MR. [Abdominal intercostal neuralgia: a forgotten cause of abdominal pain]. Ned Tijdschr Geneeskd. 2006; 150: 1909-1915.

7. Srinivasan R, Greenbaum DS. Chronic abdominal wall pain: a frequently overlooked problem. Practical approach to diagnosis and management. Am J Gastroenterol. 2002; 97: 824-830.

8. Suleiman S, Johnston DE. The abdominal wall: an overlooked source of pain. Am Fam Physician. 2001; 64: 431-438.

9. Thomson WH, Dawes RF, Carter SS. Abdominal wall tenderness: a useful sign in chronic abdominal pain. Br J Surg. 1991; 78: 223-225.

10. Gallegos NC, Hobsley M. Recognition and treatment of abdominal wall pain. J R Soc Med. 1989; 82: 343-344.

11. van Assen T, de Jager-Kievit JW, Scheltinga MR, et al. Chronic abdominal wall pain misdiagnosed as functional abdominal pain. J Am Board Fam Med. 2013; 26: 738-744.

12. Boelens OB, Scheltinga MR, Houterman S, et al. Management of anterior cutaneous nerve entrapment syndrome in a cohort of 139 patients. Ann Surg. 2011; 254: 1054-1058.

13. Boelens OB, Scheltinga MR, Houterman S, et al. Randomized clinical trial of trigger point infiltration with lidocaine to diagnose anterior cutaneous nerve entrapment syndrome. Br J Surg. 2013; 100: 217-221.

14. Boelens OB, van Assen T, Houterman S, et al. A double-blind, randomized, controlled trial on surgery for chronic abdominal pain due to anterior cutaneous nerve entrapment syndrome. Ann Surg. 2013; 257: 845-849.
15. Scheltinga MR, Boelens OB, Tjon ATWE, et al. Surgery for refractory anterior cutaneous nerve entrapment syndrome (ACNES) in children. J Pediatr Surg. 2011; 46: 699-703.

16. van Assen $\mathrm{T}$, Boelens $\mathrm{OB}$, van Eerten $\mathrm{PV}$, et al. Long-term success after an anterior neurectomy in patients with an abdominal cutaneous nerve entrapment syndrome. Surgery. 2015; 157: 137-43.

17. van Assen T, Boelens OB, van Eerten PV, et al. Surgical options after a failed neurectomy in anterior cutaneous nerve entrapment syndrome. World J Surg. 2014; 38: 3105-3111.

18. Thomson H, Francis DM. Abdominal-wall tenderness: A useful sign in the acute abdomen. Lancet. 1977; 2: 1053-1054.

19. van Assen T, Boelens OB, Kamphuis JT, et al. Construction and validation of a questionnaire distinguishing a chronic abdominal wall pain syndrome from irritable bowel syndrome. Frontline gastroenterol. 2012; 3: 288-294.

20. Greenbaum DS, Greenbaum RB, Joseph JG, et al. Chronic abdominal wall pain. Diagnostic validity and costs. Dig Dis Sci. 1994; 39: 1935-1941.

21. Brewer BJ, Golden GT, Hitch DC, et al. Abdominal pain. An analysis of 1,000 consecutive cases in a University Hospital emergency room. Am J Surg. 1976; 131: 219-223.

22. Kamin RA, Nowicki TA, Courtney DS, et al. Pearls and pitfalls in the emergency department evaluation of abdominal pain. Emerg Med Clin North Am. 2003; 21: 61-72, vi.

23. Powers RD, Guertler AT. Abdominal pain in the ED: stability and change over 20 years. Am J Emerg Med. 1995; 13: 301-303.

24. Loos MJ, Scheltinga MR, Roumen RM. Tailored neurectomy for treatment of postherniorrhaphy inguinal neuralgia. Surgery. 2010; 147: 275-281.

25. ChipSoft, ChipSoft - Electronic Health and Information System 2013: Amsterdam.

26. Abdominal wall tenderness test: could Carnett cut costs? Lancet. 1991; 337: 1134

27. Ranger I, Mehta M, Pennington M. Abdominal wall pain due to nerve entrapment. Practitioner. 1971; 206: 791-792.

28. Statistiek CBvd. Bevolkingsteller. [web page] [cited 2012; Available from: http://www.cbs. nl/nl-nl/menu/themas/bevolking/cijfers/extra/bevolkingsteller.htm.

29. Gommer A, Poos M. Achtergrondcijfers bij rangordetabellen VTV2010. [Web page] 2010 [cited 201023 september 2010]; Available from: http://www.nationaalkompas.nl/gezondheid-en-ziekte/ziekten-en-aandoeningen/ achtergrondcijfers-bij-rangordetabellen/.

30. Heij HA, Go PNMYH, Kazemier G, et al., Richtlijn voor diagnostiek en behandeling van acute appendicitis, 2010, Nederlandse vereniging voor de heelkunde: Utrecht. p. 89. 


\title{
Chapter 5
}

\author{
Anterior cutaneous nerve \\ entrapment syndrome and the \\ avoidable economic burden
}

\author{
Tijmen van Assen \\ Chrystel Perquin \\ Clazien A.M. Bouwmans \\ Percy V. van Eerten \\ Marc R. Scheltinga \\ Rudi M. Roumen
}




\section{Abstract}

Background

Doctor's delay may be substantial in patients with pain due to abdominal wall syndromes such as the anterior cutaneous nerve entrapment syndrome (ACNES). As a consequence, avoidable health care consumption prior to diagnosis is also deemed extensive. Aim of the present study was to calculate avoidable direct diagnostic and therapeutic costs and indirect productivity costs in the year preceding the diagnosis of ACNES in a patient population of a single hospital (Máxima Medical Center, $\mathrm{MMC})$.

Methods

Patients who were referred from outside the hospital's adherence area and who received the new diagnosis ACNES between September 2012 and August 2014 in MMC were eligible for the study. Study subjects were invited to complete the medical consumption questionnaire (iMCQ) and the productivity consumption questionnaire (iPCQ). Numbers of health care consumption obtained from the iMCQ were multiplied with unit cost prices to attain direct diagnostic and therapeutic costs. Productivity losses were calculated by adding up the costs of absenteeism, presenteeism and unpaid work loss.

Results

94 Patients fulfilled criteria for the new diagnosis ACNES (female $n=74,79 \%$, mean age 41 SD 17). Total direct health care costs in the year preceding the diagnosis was estimated to approximate $€ 5,400$ per patient. Extrapolation to total number of years of doctor's delay resulted in direct medical costs of $€ 16,600$ per patient. A total of $€ 13,800$ of indirect costs per patient in the year before diagnosis was calculated, leading to a total of $€ 19,200$ in the year preceding the correct diagnosis.

Conclusions

Patients with anterior cutaneous nerve entrapment syndrome are avid health care consumers in the year preceding their diagnosis. Earlier recognition will reduce unnecessary direct and indirect costs associated with the diagnostic and treatment approach.

\section{Introducion}

The cause of abdominal pain is often obscure. In an acute setting, a definitive diagnosis is uncertain in over $40 \%$ of patients with sudden onset abdominal pain ${ }^{1}$. The etiology of chronic abdominal pain is also often elusive ${ }^{2}$. A substantial number of patients treated as having chronic visceral abdominal pain may in fact suffer from chronic abdominal wall pain (CAWP) syndromes ${ }^{3-9}$.

A characteristic example of a CAWP syndrome is the anterior cutaneous nerve entrapment syndrome (ACNES). Terminal branches of thoracic intercostal nerves are somehow entrapped in layers of the abdominal wall'. An ACNES patient experiences severe neuropathic pain in a circumscript area of an anterior portion of the abdominal area. Physical examination consistently unveils a point of maximal pain. Altered skin sensibility (usually hyperesthesia) covering this painful area reflects the neuropathic character of this pain syndrome. A positive Carnett test may contribute to the diagnosis ${ }^{3,7,-12}$.

Although the entity has been recognized for over 80 years, many physicians still are unfamiliar with specifics of the syndrome ${ }^{3-9}$. If unrecognized, a series of unnecessary costly diagnostic evaluations may be ordered by general practitioners and hospital specialists ${ }^{9,10}$. For instance, one study reported that 418 diagnostic procedures were executed in 100 CAWP patients ${ }^{6}$. Similarly, a 1994 report showed that a workup accounted for 680 US\$ per CAWP patient ${ }^{2}$. Moreover, a staggering 6727 US\$ per patient was invested in futile diagnostic testing and hospital charges before arriving at the correct diagnosis in a 2001 CAWP study ${ }^{13}$. However, methodology in these somewhat outdated studies was not similar, whereas extrapolation to the Dutch system is difficult due to a different organization of health care system.

In the 'SolviMáx outpatient department for abdominal wall and groin pain syndromes', physicians are consistently confronted with ACNES patients who have also received numerous, and often unnecessary, diagnostic as well as invasive procedures before the correct diagnosis was established. Moreover, many patients reported difficulties in daily life activities and paid work, leading to sick leave and even unemployment. An analysis of avoidable costs adopting a societal perspective in an ACNES population may demonstrate the relative economic consequences of this frequently overlooked syndrome and may show the configuration of health care consumption across different parties. ${ }^{14} \mathrm{An}$ investigation may give clues for a potential scope of improved recognition of the disease. 
Aim of this present study was to assess direct medical and indirect non-medical costs due to an unrecognized CAWP syndrome in a population of ACNES patients in the final year preceding the appropriate diagnosis.

\section{Methods}

\section{General information}

A questionnaire survey was performed between September 2012 and August 2014 at the surgical department of the Máxima Medical Center (MMC), a 595-bed community hospital situated in the southern part of The Netherlands. Staff members of the surgical department of MMC have gained considerable experience in diagnosing syndromes of chronic abdominal wall pain and groin pain ever since the beginning of the new millennium ${ }^{15-26}$. As a consequence, a surgical subdepartment termed 'SolviMáx' (Center of Excellence for Abdominal Wall and Groin Pain) was initiated that evaluates a growing number of patients yearly by a team of surgical and medical experts. Most referred patients are resided outside of the MMC hospital's adherence area.

\section{Patient selection}

SolviMáx physicians have experienced that potential ACNES patients living close to the MMC are possibly presented with a limited delay as a relative high awareness within the $\mathrm{MMC}$ adherence area is hypothesized. In contrast, a considerable doctor's delay is present in many patients who are referred from outside the adherence area. Associated health care costs in patients from these other Dutch areas will more reliably reflect the current Dutch daily practice. Moreover, extrapolation to areas other than MMC's is also more realistic as a comparable situation will also prevail in many other countries.

During a two years time period (September 2012 - August 2014), consecutively referred patients from outside the $\mathrm{MMC}$ adherence area who received the new diagnosis ACNES in the MMC were asked to participate in the current study. Our adherence area is defined as the total group of general practitioners who would, based on prior contacts and geography, refer patients to the MMC. Accurate figures on MMC adherence were obtained from a standard market analysis (Kiwa Carity B.V., Utrecht, The Netherlands). Study patients were invited to complete the iMTA Medical Consumption Questionnaire $(i \mathrm{MCQ})^{27}$. Exclusion criteria were a prior diagnosis (and treatment) of ACNES, an alternative condition associated with abdominal pain or a second opinion for patients in whom the diagnosis ACNES was already made or considered previously. Patients that were not able to properly read or understand the Dutch language as well as patients not willing to participate were not included as well. Symptom duration and age were not defined as exclusion criteria.

\section{Diagnostic criteria of ACNES}

Diagnosing ACNES occurred during either the first, the second or the third outpatient department visit. As a consequence, some patients received the questionnaire after the first visit while others after their next visit.

The diagnosis ACNES was confirmed or deemed highly likely if the following set of signs and symptoms were met, as previously published ${ }^{16,17}$ :

1. Abdominal pain, and

2. Site of abdominal tenderness with a small ( $<2 \mathrm{~cm} 2$, "finger tip") area of maximal intensity that is situated within the lateral boundaries of the rectus abdominis muscle, and

3. Tenderness increases by abdominal muscle tensing using the Carnett's test. The point of maximal pain is localized using a palpating index finger. Muscles are tensed as the patient lifts the head. If tenderness increases, the origin of the pain is most likely located in the abdominal wall ${ }^{5,10}$, and

4. A positive pinch test reflecting skin sensory disturbances covering the area of maximal pain, and

5. Normal laboratory analysis and normal imaging (if previously performed), and

6. Pain reduction following infiltration using $10 \mathrm{~mL}$ of $1 \%$ lidocaine.

During follow up, no alternative diagnoses possibly explaining the abdominal pain emerged in any of the patients.

\section{Cost measurement}

This study adopted a societal perspective including the following costs: (1) first line medical costs, (2) medication costs, (3) home care costs, (4) hospital costs including cost of outpatient specialist visits, diagnostics, (day care) treatment, surgery and hospitalization, and (5) indirect non-medical costs of productivity losses. Medical consumption and productivity losses were measured using the Medical Consumption 
Questionnaire ( $i \mathrm{MCQ}$ ) and the Productivity Cost Questionnaire ( $i \mathrm{PCQ})$ as developed by the institute for Medical Technology Assessment (www.imta.nl) ${ }^{28-30}$. These questionnaires are recommended in the manual for economic evaluations in health care (CVZ, College voor zorgverzekeringen; Health Care Insurance Board). These generic questionnaires are developed as patient self-reporting tools. ${ }^{31}$ For the purpose of this study, questionnaires were adjusted in such a way that only medical consumption and production losses as a consequence of the abdominal complaints due to ACNES were asked. Each questionnaire is preceded by general questions determining sociodemographics of the respondent without revealing the patient's identity. Completing both questionnaires approximately requires 30-45 minutes. By completing the questionnaire, patients automatically signed for informed consent.

\section{Direct medical costs}

The $i \mathrm{MCQ}$ includes 31 questions related to medical consumption including numbers of first line health care visits, medication use, hospital outpatient department visits, diagnostic evaluations, hospital daycare, inpatient care and surgery. In the original version of the $i \mathrm{MCQ}$, a 3 months recall period is used as a means to generate reliable information ${ }^{32,33}$. As ACNES patients usually face a longer doctor's delay and health consumption can fluctuate over a longer period, a recall period of 1 year rather than 3 months was deemed more representative ${ }^{17}$.

Unit cost prices of first line care were derived from reference lists of the manual for economic evaluations in health care from the Health Care Insurance Board (CVZ) ${ }^{31}$. Medication costs were calculated using standard medication prices as described by the Health Care Insurance Board (CVZ), online available on www.medicijnkosten.nl ${ }^{34}$. If applicable, the costs were based on the generic name of the product ${ }^{34,35}$. The cheapest variant was chosen for calculations. Delivery tariff for not freely available drugs was added applying a standard fee of $€ 6$ per prescribed drug per period of 3 months. Unit prices for radiologic and other diagnostic procedures were taken from the NZa (Dutch Healthcare Authority) which are assumed to provide a good indication of the actual costs ${ }^{36}$. Costs of most surgical interventions are available within prices of health care products (DBC, Diagnosis Treatment Combination, www.opendisdata.nl) ${ }^{37}$. Outpatient department visits, diagnostics, therapies and hospitalization are included into these products. Only the portion responsible for the health unit of interest (surgery) was calculated according to MMC's product profiles. All costs were indexed to the year of 2013 using data from Statistics Netherlands (CBS). Unit costs prices of hospital health care (diagnostic procedures, (surgical) treatment and inpatient care) derived from the different resources were re-checked for validity by MMC's financial department participating in a benchmark together with comparable type of hospitals.

Obtained numbers of health care consumption were multiplied by the calculated unit cost prices. The portion of patients consuming a health care component was calculated as well as total number of units. Subsequently, total costs per health care section and finally overall total medical costs were obtained.

\section{Indirect non-medical costs}

The $i \mathrm{PCQ}$ is used for the measurement of indirect non-medical costs due to productivity losses related to the abdominal complaints. The $i$ PCQ consists of 3 modules adding up to a total of 12 questions. The modules include productivity of paid work as a consequence of absenteeism, productivity loss of paid work without absenteeism (presenteeism) and productivity loss of unpaid work. In the questionnaire, standard recall period of 4 weeks is handled as longer periods lead to inaccuracy in these self-reports ${ }^{38}$. Costs were, were extrapolated to the total duration of complaints, with a maximum of 52 weeks ( 1 year).

Productivity losses at a paid job as a consequence of absenteeism were calculated in two situations; short absenteeism ( $\leq 4$ weeks) and long absenteeism ( $>4$ weeks). For patients with long absenteeism, the 'friction cost method' was used, according to the Dutch guidelines of economic evaluations in healthcare ${ }^{31,39,40}$. This method estimates production losses of long-term absence from work only for the period required to replace the employee, starting from the beginning of subject's sick leave ('friction period'). In absence of reliable information about the duration of a vacancy for the year of 2013, a 2011 friction period of 116 days ( 17 weeks) was used ${ }^{41}$. If the friction period expired more than a year ago (in the case of a patient who is sick for work at least 69 weeks $(52+17)$ ), no costs due to productivity losses were calculated. For the calculation of the productivity losses, age specific production costs were used.

Productivity loss of paid work without absenteeism (presenteeism) was calculated as number of hours of productivity loss (as a product of daily working hours and number of days with complaints) multiplied with a factor of efficiency loss (between 0 and 1) during these days. The lost productivity hours were multiplied with mean productivity costs of different age groups to calculate costs of presenteeism ${ }^{31}$. Productivity loss of unpaid work was calculated by multiplying lost hours of unpaid work with the standard costs of domestic help $(€ 12,50)$. Total costs for all three types of production losses are displayed as mean and total cost. 


\section{Data collection and analysis}

All analyses were performed with the SPSS 20.0 program for Mac OS X file. Baseline characteristics were presented as percentages or mean (SD) as appropriate. Total costs for the study population are displayed as rounded costs. Missing items were excluded in individual health care units. For total cost calculation, missings were counted as a zero.

\section{Results}

\section{Demographics}

In the almost two year study period, 1267 unique patients visiting the SolviMáx outpatient department were evaluated for a chronic abdominal wall or inguinal pain syndrome. A total of 501 patients were diagnosed with ACNES, and two-thirds $(n=329)$ were referred from outside the MMC's adherence area. Some of these patients did not participate because of a language barrier or were not interested to participate. From the remaining eligible patients, an unknown number was not invited to complete the questionnaire, likely due to time restraints associated with an exceedingly busy practice. As a consequence, a total of 169 patients were invited to complete the questionnaire. Complete data sets of 94 patients were returned and analysed (56\%).

Most patients were female $(\mathrm{n}=74,79 \%)$ as also found in other studies ${ }^{16,17,42,43}$. Age ranged from 13 to 78 years (mean 41 yr., SD 17). The majority ( $\mathrm{n}=74,79 \%)$ suffered from ACNES for more than one year. The average duration of symptoms was almost 4.5 years (53 months, SD 82, range 2 - 576). Respondents with paid work ( $n=59$ ) were employed for on average of 30 hours a week (SD 12, rage 5-60).

\section{Direct medical costs}

Detailed information on type of medical consumption and associated costs are presented in table 1. Total health care costs in the year preceding the diagnosis was estimated to on average $€ 5,400$ per patient.

Most patients visited a general practitioner (GP) in the year preceding diagnosis. Some $90 \%$ reported at least one GP visit (85/94). These patients had paid an average of 7 visits. Less than half (41\%) of the study population was referred to a physical therapist (PT). These patients reported on average 15 PT visits. Total cost of first line care added up to $€ 650$ per patient. Consumption of almost 90.000 medication pills resulted in some
$€ 16,300$ of additional costs including $€ 1,700$ of delivery costs (on average $€ 170$ per patient). Only 2 patients did not use pain medication. Painkillers, laxatives, tranquilizers and antidepressants were frequently used (59 different types of drugs).

Approximately half of the patients reported outpatient hospital visits to gastroenterologists and surgeons in the pre-diagnosis year. About thirty percent of the respondents also reported visits to other specialists such as pain doctors, internists and gynaecologists. Of 10 adolescents who were included in this analysis, 5 were evaluated by a paediatrician. Total cost of outpatient visits accounted for $€ 670$ per patient.

A substantial number of imaging studies including colonoscopies ( $\mathrm{n}=51), \mathrm{CT}$ scans $(\mathrm{n}=54)$ and ultrasounds $(\mathrm{n}=95)$ indicate the persistent search for a visceral cause of the pain. The majority of day-care hospital treatments were associated with pain therapy and accounted for $€ 1,010$ per patient. More than one-third of the patients (37\%) was hospitalised (on average 6 days per patient) in the year prior to diagnosis (on average $€ 1,130$ per patient). A total of 37 invasive surgical procedures were executed because of the abdominal pain including diagnostic laparoscopies $(n=7)$, appendectomies $(n=6)$, umbilical hernia repair $(n=8)$, cicatricial hernia repair $(n=4)$, scar tissue correction $(n=2)$, rib resection $(n=1)$, abscess incision $(n=1)$, uterus extirpation $(n=1)$, ovarian removal $(n=4)$ and nerve exploration $(n=3)$. Associated costs with these interventions added up to a total of on average $€ 460$ per patient.

Twenty of 74 patients suffering from pain for more than one year spontaneously stated in the comments section that the majority of health care was consumed prior to the study's one-year recall period justifying an extrapolation of the calculated costs. In all patients having ACNES pain for more than one year $(n=74,79 \%)$, costs over this year were multiplied by the total duration of complaints in years. This extrapolation resulted to an estimated total health care costs of $€ 1,500,000$, on average $€ 16,600$ per patient (range, $€ 470$ - €98,100). 
Table 1 Medical costs in a series of patients in the year prior to the diagnosis anterior cutaneous nerve entrapment syndrome (ACNES)

\begin{tabular}{lrrrrr} 
Component & $\begin{array}{r}\text { \% of n=94 } \\
\text { consuming } \\
\text { component } \\
\text { (missing) }\end{array}$ & $\begin{array}{c}\text { Mean no of } \\
\text { units per } \\
\text { censuming }\end{array}$ & $\begin{array}{r}\text { Total number } \\
\text { of units }\end{array}$ & $\begin{array}{r}\text { Unit costs } \\
(€)\end{array}$ & $\begin{array}{r}\text { Total costs } \\
(€)\end{array}$ \\
\hline First line care (visits) & $97(0)$ & & & & \\
\hline Physiotherapist & $41(0)$ & 15 & 573 & 39.13 & 22,400 \\
General practitioner & $90(0)$ & 7 & 594 & 30.44 & 18,200 \\
Psychology & $23(0)$ & 5 & 106 & 86.96 & 9,200 \\
Company doctor & $32(0)$ & 5 & 164 & 30.44 & 5,000 \\
Social worker & $7(0)$ & 5 & 37 & 70.66 & 2,600 \\
Homeopathy & $16(0)$ & 5 & 74 & 39.13 & 2,900 \\
Dietician & $17(0)$ & 3 & 40 & 29.35 & 1,200 \\
Total (mean per patient): & & & & & $\mathbf{6 1 , 5 0 0 ( 6 5 0 )}$
\end{tabular}

\begin{tabular}{|c|c|c|c|c|c|}
\hline Home care (hours) & $6(0)$ & & & & \\
\hline Domestic help & $5(4)$ & 142 & 708 & 26.09 & 18,500 \\
\hline Nursing & 1(4) & 260 & 260 & 70.66 & 18,400 \\
\hline Total (mean per patient): & & & & & $36,800(390)$ \\
\hline Medication (tablets/units) & $98(0)$ & & & & \\
\hline Medication & & & 88.188 & & 14,600 \\
\hline Delivery fee & & & & & 1,700 \\
\hline Total (mean per patient): & & & & & $16,300(170)$ \\
\hline Specialist (visits) & $95(0)$ & & & & \\
\hline Surgeon & $55(2)$ & 4 & 192 & 78.26 & 15,000 \\
\hline Gastroenterologist & 50 (2) & 4 & 170 & 78.26 & 13,300 \\
\hline Pain specialist & $32(2)$ & 4 & 134 & 78.26 & 10,500 \\
\hline Internal medicine & 29 (2) & 3 & 88 & 78.26 & 6,900 \\
\hline Gynaecologist & 30 (2) & 3 & 79 & 78.26 & 6,200 \\
\hline Urologist & $13(2)$ & 4 & 49 & 78.26 & 3,800 \\
\hline Paediatrician & $5(2)$ & 8 & 39 & 78.26 & 3,100 \\
\hline Neurologist & $9(2)$ & 4 & 28 & 78.26 & 2,200 \\
\hline Other specialisms $(n=5)$ & 1-3 (2) & & 27 & 78.26 & 2,100 \\
\hline Total (mean per patient): & & & & & $63,100(670)$ \\
\hline Diagnostic tests (units) & $78(1)$ & & & & \\
\hline Colonoscopy & 29 (2) & 2 & 51 & 368.41 & 18,800 \\
\hline MRI-scan & 34 (2) & 1 & 46 & 200.73 & 9,200 \\
\hline CT-scan & $42(2)$ & 1 & 54 & 164.01 & 8,900 \\
\hline Echography & $45(2)$ & 2 & 95 & 52.63 & 5,000 \\
\hline Gastroscopy & $11(2)$ & 1 & 13 & 368.41 & 4,800 \\
\hline Triple faeces test & $7(2)$ & 2 & 14 & 128.00 & 1,800 \\
\hline$X$-ray & $14(2)$ & 2 & 28 & 46.50 & 1,300 \\
\hline
\end{tabular}

\begin{tabular}{|c|c|c|c|c|c|}
\hline Cystoscopy & $5(2)$ & 2 & 9 & 102.81 & 900 \\
\hline CT-colonography & $3(2)$ & 1 & 4 & 164.01 & 700 \\
\hline Blood tests & $11(2)$ & 2 & 22 & 22.87 & 500 \\
\hline Capsule endoscopy & $3(2)$ & 1 & 3 & 139.52 & 400 \\
\hline Other incidental diagnostic tests $(n=8)$ & $1-2(2)$ & & 13 & & 1,800 \\
\hline Total (mean per patient): & & & & & $54,100(580)$ \\
\hline Hospital day care treatment & $59(1)$ & & & & \\
\hline Nerve blocks & 30 (2) & 3 & 83 & 551.35 & 45,800 \\
\hline General pain treatment & $12(2)$ & 3 & 38 & 551.35 & 21,000 \\
\hline $\begin{array}{l}\text { Transcutaneous Electrical Nerve } \\
\text { Stimulation (TENS) }\end{array}$ & $9(2)$ & 4 & 35 & 551.35 & 20,100 \\
\hline Emergency dept. visit & $18(2)$ & 2 & 33 & 164.14 & 5,400 \\
\hline Constipation & 2(2) & 6 & 11 & 215.87 & 2,400 \\
\hline Iontoforesis & 1 (2) & 1 & 1 & 551.35 & 600 \\
\hline Total (mean per patient): & & & & & $95,300(1,010)$ \\
\hline Out of hospital treatment (days) & $4(1)$ & & & & \\
\hline Revalidation clinic & $4(1)$ & 18 & 70 & 369.58 & 25,900 \\
\hline Total (mean per patient): & & & & & $25,900(280)$ \\
\hline Hospitalisation (nights) & $37(1)$ & 6 & 214 & 496.76 & 106,300 \\
\hline Total (mean per patient): & & & & & $106,300(1,130)$ \\
\hline Surgery & 31 (1) & & & & \\
\hline Umbilical hernia repair & 7 (2) & 1 & 8 & 1188.18 & 9,500 \\
\hline Appendectomies & 7 (2) & 1 & 6 & 1282.28 & 7,700 \\
\hline Ovariectomy & $3(2)$ & 1 & 4 & 1844.59 & 7,400 \\
\hline Cicatricial hernia rep. & $3(2)$ & 1 & 4 & 1759.67 & 7,000 \\
\hline Diagnostic laparoscopy & $8(2)$ & 1 & 7 & 875.40 & 6,100 \\
\hline Neurectomy & $3(2)$ & 1 & 3 & 882.84 & 2,600 \\
\hline Uterus extirpation & 1 (2) & 1 & 1 & 1844.59 & 1,800 \\
\hline Rib resection & 1 (2) & 1 & 1 & 1005.47 & 1,000 \\
\hline Abscess incision & 1 (2) & 1 & 1 & 165.03 & 200 \\
\hline Scar tissue resection & 2 (2) & 1 & 2 & 56.00 & 100 \\
\hline Total (mean per patient): & & & & & $43,500(460)$ \\
\hline Total overall: & & & & & $€ 503,100$ \\
\hline $\begin{array}{l}\text { Total per patient: } \\
\text { (range) }\end{array}$ & & & & & $\begin{array}{r}€ 5.400 \\
(150-29.300)\end{array}$ \\
\hline
\end{tabular}




\section{Indirect non-medical costs}

The different types of productivity losses and their costs are presented in figure 1. A total of $€ 13,800$ per patient of indirect costs due to productivity losses was calculated in the year preceding diagnosis, despite a relatively high number of missings of 'cost of unpaid work loss' in particular $(\mathrm{n}=17)$.

More than half of the respondents with paid work $(58 \%, 34 / 59)$ reported sick leave prior to receiving the diagnosis ACNES. The majority $(68 \%, 23 / 34)$ reported longterm absence from work (e.g. more than 4 weeks; mean 45 weeks, SD 37, range 4 - 123 weeks). The other 11 employees reported shorter absence. Cost of absence from work was estimated at $€ 7,500$ per patient with a paid job in the year before diagnosis.

$63 \%$ (36/59) of the patients with paid work did not report absence from work during the complete recall-period. However, 31 of these 36 (86\%) stated to have been at work while suffering from abdominal pain (range 1-21 working days in 4 weeks). 28 of 31 employees reported to have experienced loss of productivity due to the complaints (10$70 \%$ ), leading to $€ 5,100$ productivity loss per respondent with a paid job in the year preceding diagnosis. In the study population, 57 patients reported to have loss of unpaid work, on average 17 hours a week per patient. In the year before diagnosis, a mean $€ 9,800$ unpaid work loss per patient who reported this type of work loss was estimated. The total of ACNES in the year preceding the diagnosis were estimated at $€ 19,200$ per patient of which $28 \%$ are direct medical costs and $72 \%$ are indirect non-medical costs. Most of these cost can be assigned as avoidable cost.
Figure 1 Costs associated with loss of productivity due to abdominal pain in ACNES (€).

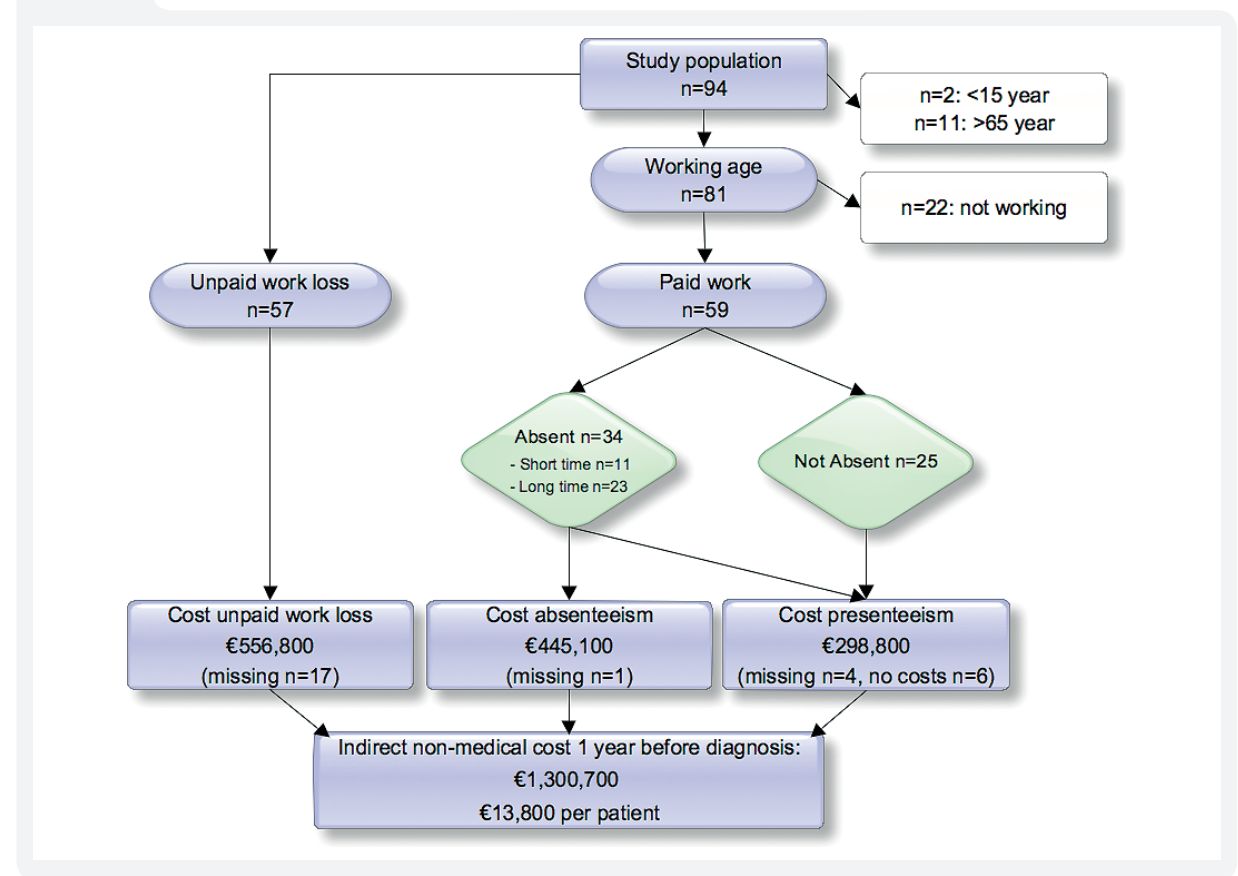

\section{Discussion}

This study demonstrates that the presence of chronic abdominal pain due to anterior cutaneous nerve entrapment syndrome (ACNES) is associated with substantial direct medical and indirect non-medical costs in the year preceding the diagnosis. Assuming that the current study population represents the actual Dutch situation, medical costs added up to a mean $€ 5,400$ per patient. Adopting a societal perspective including productivity losses, an average $€ 19,200$ per patient was found. Most of these costs may be avoidable.

Some older reports studying health care costs in ACNES patients focused on specific portions of the health care process ${ }^{2,6}$. For instance, Thompson investigated medical costs prior to trigger point injections for abdominal wall pain syndrome in $2001^{13}$. In that study, costs associated with all diagnostics and treatments were similar compared to the current study (6.727 US\$ $\approx € 5,367$ versus $€ 5,400$ per patient). However, the present study 
only focused on costs in the final one year before diagnosis. Total medical costs were more than three times higher $(€ 16,600)$ following extrapolation to total years of pain. Moreover, we also identified a substantial amount of indirect costs in the year before diagnosis as a consequence of the invalidating character of the disease. The most plausible explanation of these high costs is twofold. First, the high intensity of neuropathic pain in these patients leads to high medical consumption and productivity losses. Second, unawareness of ACNES among physicians leads to unnecessary diagnostics and treatments resulting in a delay of the appropriate diagnosis and treatment.

Not a single specific health care provider is to blame for the ignorance concerning ACNES. Most patients participating in the present study visited a great variety of doctors. In the present-day environment of superspecialisation, physicians progressively retire into their own back yard ${ }^{44}$. As a consequence, syndromes including ACNES that are not clearly adopted by a certain specialism stand a chance of being ignored by all neighbouring specialists. Gastroenterologists, internists, gynaecologists, paediatricians and surgeons need to be educated on the clinical significance of the abdominal wall. Moreover, ACNES as a differential diagnostic alternative deserves a more prominent place in the curriculum of future general practitioners.

It may seem that an improved recognition and understanding of ACNES can prevent many unnecessary diagnostics and treatments thus reducing medical costs. Moreover, a frustrating patient's quest of years may be shortened. Once diagnosed, ACNES patients in the current study undergo a simple treatment protocol that was recently developed ${ }^{16-18}$, ${ }^{42}, 43$. In an imaginary population of 100 patients, a regimen of $1-3$ abdominal wall injections is long term effective in one-thirds ${ }^{17,43}$. Estimated costs associated with such an approach (including visits to GP, surgeon and injection treatment) based on similar unit cost prices adds up to approximately $€ 1,400$ per patient. For patients not responding to this injection regimen, a surgical exploration including transection of the nerve endings (neurectomy) was effective in a randomized double blind surgical trial ${ }^{18}$. Such a neurectomy performed in the remaining 67 patients after a conservative treatment cost approximately $€ 2,700$ per patient. Based on a published $61 \%$ long term success rate in this population, some 26 patients remain with persistent pain who are offered a second surgical exploration $(€ 4,700)^{42}$. As a $66 \%$ success rate is attained, some 9 of the original 100 patients possibly do not benefit from this three-step treatment protocol ${ }^{43}$. Conversely, over $90 \%$ of a hypothetical ACNES population is diagnosed and successfully treated at the expense of approximately $€ 2,800$ of medical costs per patient. The alternative is an expensive path associated with on-going chronic pain. As demonstrated in this study, the average ACNES patient suffered for over 4 years. Direct and indirect costs exceeded $€ 19,000$ per patient, an amount that is 7 times higher compared to the cost of the proposed treatment regimen. Therefore, improved awareness and appropriate treatment of ACNES lead to serious savings.

The current study focused on the burden of ACNES in terms of cost to society. However, results also demonstrate a substantial inability to fulfill normal daily activities in terms of high rates of sick leave and efficiency losses of paid work in ACNES patients. Moreover, it may be hypothesized that a delay in diagnosis and inappropriate treatment will also seriously affect quality of life. Such studies are recommended to attain a complete evaluation of health status in ACNES patients ${ }^{45}$.

The current study may harbour flaws. All participating physicians $(n=5)$ were instructed to check patients for eligibility, but the questionnaire was completed anonymously. As a consequence, correctness of data could not be verified whereas reasons for refusal to participate were unclear. Furthermore, some potential patients were possibly declined participation due to time restraints associated with a busy practice, leading to the $51 \%$ inclusion rate among eligible patients. A 56\% response rate is comparable to other rates obtained from mail surveys published in medical journals (approximately $60 \%)^{46}$. Characteristics including age and gender of the study population $(\mathrm{n}=94$, female $79 \%$, mean age 41 SD 17 , range $13-78$ ) and the overall eligible population ( $n=329$ : female $78 \%$, mean age 40 SD 18, range 8 - 81) were identical indicating that a representative group participated in the current study. Another potential flaw is a prolonged recall period (12 rather than 3 months) that was used for the measurement of medical consumption. Therefore, recall bias may have played a role. However, this type of bias usually leads to an underestimation of $\operatorname{costs}^{33}$. Furthermore, questions related to medication use were difficult to handle for some patients as previously reported ${ }^{30}$. However, missing items also lead to an underestimation of the costs. The number of missing data for calculation of indirect non-medical costs due to productivity losses, in particular the module unpaid work loss, was substantial. Again however, this omission also leads to underestimated costs.

Based on a recently calculated 57:100.000 ACNES incidence rate, costs of illness at a given moment may be approximated ${ }^{47}$. If extrapolated to the Dutch population (2013, 16.800.000 inhabitants), some 9.500 new ACNES patients may be identified yearly ${ }^{48}$. 
On the basis of these assumptions, total yearly costs associated with ACNES in The Netherlands are calculated at $€ 182.4$ million ( $€ 51.3$ million direct medical costs and $€ 131.1$ million indirect non-medical costs). In this calculation, it is also assumed that all newly diagnosed ACNES patients experience the same burden of disease, which is not necessarily a realistic picture. However, the incidence rate calculation in one other study was only based on ACNES patients that consulted an outpatient department or the emergency department, indicating a population with severe complaints ${ }^{47}$. If all these patients would undergo a diagnostic and treatment regimen as proposed $(€ 2,800$ per patient for diagnosis and treatment), direct medical cost is calculated at $€ 26.6$ million per year. An indirect non-medical cost calculation cannot be determined reliably, but a maximum half a year treatment period (including queue time prior to first evaluation and the period between treatment steps) is reasonable. According to similar productivity losses as calculated in the studied population, an estimated yearly cost reduction of €80100 million is reasonable in the Dutch situation.

\section{Conclusion}

ACNES patients are avid health care consumers in the final year preceding their diagnosis. Earlier recognition and treatment of ACNES will result in a substantial reduction of direct medical and indirect non-medical costs.

\section{References}

1. Graff LGt, Robinson D. Abdominal pain and emergency department evaluation Emerg Med Clin North Am. 2001; 19: 123-136.

2. Greenbaum DS, Greenbaum RB, Joseph JG, et al. Chronic abdominal wall pain. Diagnostic validity and costs. Dig Dis Sci. 1994; 39: 1935-1941.

3. Abdominal wall tenderness test: could Carnett cut costs? Lancet. 1991; 337: 1134

4. Applegate WV. Abdominal cutaneous nerve entrapment syndrome. Surgery. 1972; 71: 118-124.

5. Carnett J. Intercostal neuralgia as a cause of abdominal pain and tenderness. Surg Gynecol Obstet. 1926; 42: 8.

6. Hershfield NB. The abdominal wall. A frequently overlooked source of abdominal pain J Clin Gastroenterol. 1992; 14: 199-202.

7. Ranger I, Mehta M, Pennington M. Abdominal wall pain due to nerve entrapment. Practitioner. 1971: 206: 791-792.

8. van Assen T, de Jager-Kievit JW, Scheltinga MR, et al. Chronic abdominal wall pain misdiagnosed as functional abdominal pain. J Am Board Fam Med. 2013; 26: 738-744.

9. Srinivasan R, Greenbaum DS. Chronic abdominal wall pain: a frequently overlooked problem. Practical approach to diagnosis and management. Am J Gastroenterol. 2002; 97: $824-830$

10. Applegate WV. Abdominal Cutaneous Nerve Entrapment Syndrome (ACNES) A Commonly Overlooked Cause of Abdominal Pain. Perm J. 2002; 6: 20-27.

11. Gallegos NC, Hobsley M. Recognition and treatment of abdominal wall pain. J R Soc Med 1989; 82: 343-344.

12. Thomson H, Francis DM. Abdominal-wall tenderness: A useful sign in the acute abdomen. Lancet. 1977; 2: 1053-1054.

13. Thompson C, Goodman R, Rowe WA. Abdominal wall syndrome, a costly diagnosis of exclusion. Gastroenterol. 2001; 120: A637.

14. Maniadakis N, Gray A. The economic burden of back pain in the UK. Pain. 2000; 84: $95-$ 103.

15. van Assen T, Boelens OB, Kamphuis JT, et al. Construction and validation of a questionnaire distinguishing a chronic abdominal wall pain syndrome from irritable bowel syndrome. Frontline gastroenterol. 2012; 3: 288-294. 
16. Boelens OB, van Assen T, Houterman S, et al. A double-blind, randomized, controlled trial on surgery for chronic abdominal pain due to anterior cutaneous nerve entrapment syndrome. Ann Surg. 2013; 257: 845-849.

17. Boelens OB, Scheltinga MR, Houterman S, et al. Management of anterior cutaneous nerve entrapment syndrome in a cohort of 139 patients. Ann Surg. 2011; 254: 1054-1058.

18. Boelens OB, Scheltinga MR, Houterman S, et al. Randomized clinical trial of trigger point infiltration with lidocaine to diagnose anterior cutaneous nerve entrapment syndrome. Br JSurg. 2013; 100: 217-221.

19. Loos MJ, Roumen RM, Scheltinga MR. Chronic sequelae of common elective groin hernia repair. Hernia. 2007; 11: 169-173

20. Loos MJ, Roumen RM, Scheltinga MR. Classifying post-herniorrhaphy pain syndromes following elective inguinal hernia repair. World J Surg. 2007; 31: 1760-1765; discussion 1766-1767.

21. Loos MJ, Scheltinga MR, Mulders LG, et al. The Pfannenstiel incision as a source of chronic pain. Obstet Gynecol. 2008; 111: 839-846.

22. Loos MJ, Scheltinga MR, Roumen RM. Surgical management of inguinal neuralgia after a low transverse Pfannenstiel incision. Ann Surg. 2008; 248: 880-885.

23. Loos MJ, Scheltinga MR, Roumen RM. Tailored neurectomy for treatment of postherniorrhaphy inguinal neuralgia. Surg. 2010; 147: 275-281.

24. Loos MJ, Verhagen T, Scheltinga MR, et al. A randomised controlled trial of injection therapy versus neurectomy for post-herniorrhaphy inguinal neuralgia: rationale and study design. Hernia. 2010; 14: 593-597.

25. Roumen RM, Scheltinga MR. [Abdominal intercostal neuralgia: a forgotten cause of abdominal pain]. Ned Tijdschr Geneeskd. 2006; 150: 1909-1915.

26. Scheltinga MR, Boelens OB, Tjon ATWE, et al. Surgery for refractory anterior cutaneous nerve entrapment syndrome (ACNES) in children. J Pediatr Surg. 2011; 46: 699-703.

27. Rotterdam EU. institute for Medical Technology Assessment. 2014 [cited 2014; Available from: http://www.bmg.eur.nl/imta/.

28. iMTA ifMTA, Productivity Cost Questionnaire, 2012, Erasmus University Rotterdam.

29. iMTA ifMTA, Medical Consumption Questionnaire, 2012, Erasmus University Rotterdam.

30. Bouwmans C, De Jong K, Timman R, et al. Feasibility, reliability and validity of a questionnaire on healthcare consumption and productivity loss in patients with a psychiatric disorder (TiC-P). BMC health services research. 2013; 13: 217.
31. Hakkaart- van Roijen L, Tan SS, Bouwmans CAM. Manual for costing research [in Dutch] 3 ed2010: Health care insurance board. 127.

32. Goossens ME, Rutten-van Molken MP, Vlaeyen JW, et al. The cost diary: a method to measure direct and indirect costs in cost-effectiveness research. J Clin Epidemiol. 2000 53: 688-695.

33. van den Brink M, van den Hout WB, Stiggelbout AM, et al. Self-reports of health-care utilization: diary or questionnaire? Int J Tech Assess Health Care. 2005; 21: 298-304.

34. Zorginstituut N. Medicijnkosten. [Web page] 2014; Available from: http://www. medicijnkosten.nl.

35. Zorginstituut N. Farmacotherapeutisch Kompas. [Web page] 2014; Available from: http:// www.farmacotherapeutischkompas.nl/default.asp.

36. Zorgautoriteit. Nederlandse Zorgautoriteit. [Web page] 2014; Available from: http://www. nza.nl.

37. Opendisdata. [Web page] 2014; Available from: http://www.opendisdata.nl.

38. Severens JL, Mulder J, Laheij RJ, et al. Precision and accuracy in measuring absence from work as a basis for calculating productivity costs in The Netherlands. Soc Sci Med. 2000; 51: $243-249$.

39. Brouwer WB, Koopmanschap MA. The friction-cost method : replacement for nothing and leisure for free? PharmacoEconomics. 2005; 23: 105-111.

40. Koopmanschap MA, Rutten FF, van Ineveld BM, et al. The friction cost method for measuring indirect costs of disease. J Health Econ. 1995; 14: 171-189.

41. Pilgram K, Vacatures in Nederland 2011 - De vacaturemarkt en personeelswerving in beeld, U. Werknemersverzekeringen, Editor 2012, UWV WERKbedrijf. p. 74.

42. van Assen T, Boelens OB, van Eerten PV, et al. Long-term success following an anterio neurectomy in patients with an abdominal cutaneous nerve entrapment syndrome. Surgery. 2015; 157: 137-43.

43. van Assen T, Boelens OB, van Eerten PV, et al. Surgical options after a failed neurectomy in anterior cutaneous nerve entrapment syndrome. World J Surg. 2014; 38: 3105-3111.

44. St Peter RF, Reed MC, Kemper P, et al. Changes in the scope of care provided by primary care physicians. N Engl J Med. 1999; 341: 1980-1985.

45. Skevington SM, Lotfy M, O'Connell KA, et al. The World Health Organization's WHOQOLBREF quality of life assessment: psychometric properties and results of the international field trial. A report from the WHOQOL group. Qual Life Res. 2004; 13: 299-310. 
46. Asch DA, Jedrziewski MK, Christakis NA. Response rates to mail surveys published in medical journals. J Clin Epidemiol. 1997; 50: 1129-1136.

47. van Assen T, Brouns JAGM, Scheltinga MR, et al. Incidence of abdominal pain due to the anterior cutaneous nerve entrapment syndrome in an emergency department. Scand J Trauma Resusc Emerg Med. 2015; 23: 19.

48. Statistiek CBvd. Bevolkingsteller. [web page] [cited 2012; Available from: http://www.cbs. nl/nl-nl/menu/themas/bevolking/cijfers/extra/bevolkingsteller.htm. 


\title{
Chapter 6
}

\author{
A double blind, randomized, \\ controlled trial on surgery for \\ chronic abdominal pain due to \\ anterior cutaneous nerve \\ entrapment syndrome
}

\section{Oliver B. Boelens \\ Tijmen van Assen \\ Saskia Houterman \\ Marc R. Scheltinga \\ Rudi M. Roumen}




\section{Abstract}

Objective

To clarify the role of a surgical neurectomy on pain in refractory patients following conservatively treated ACNES.

\section{Background}

Anterior cutaneous nerve entrapment syndrome (ACNES) is hardly ever considered in the differential diagnosis of chronic abdominal pain. Treatment is usually conservative. However, symptoms are often recalcitrant.

Methods

Patients $>18$ years diagnosed with ACNES were randomized to receive a neurectomy or a sham procedure via an open surgical procedure in day care. Both patient and principal investigator were blinded to the nature of surgery. Pain was recorded using a Visual Analogue Scale (VAS: 1-100 mm) and a Verbal Rating Scale (VRS 0-5, $0=$ no pain, $5=$ severe pain) prior to surgery and 6 weeks postoperatively. A reduction of at least $50 \%$ of VAS and/or 2 points of VRS was considered a 'successful response'.

Results

Forty-four patients were randomized between August 2008 and December 2010 (39 females, median age 42 years, both groups $n=22$ ). In the neurectomy group, 16 patients reported a successful pain response. In contrast, significant pain reduction was obtained in 4 patients in the sham group $(\mathrm{p}=0.001)$. Complications associated with surgery were hematoma ( $\mathrm{n}=5$, conservative treatment), infection (antibiotic and drainage, $\mathrm{n}=1)$ and worsened pain $(\mathrm{n}=1)$.

\section{Conclusion}

Neurectomy of the intercostal nerve endings at the level of the abdominal wall is an effective surgical procedure for pain reduction in ACNES patients who failed to respond to a conservative regimen.

\section{Introduction}

Chronic abdominal pain (CAP) is a diagnostic challenge for both general physicians and specialists. It often occurs that a definitive diagnosis is not attained, even after extensive evaluation including laboratory tests and multimodal imaging. Interestingly, pain is found to originate from the abdominal wall rather than from visceral organs in up to $30 \%$ of patients with $\mathrm{CAP}^{1-4}$. Although a patient is seldom able to discriminate between the two sources of pain, a simple tool such as Carnett's testing contributes to a distinction between the two varieties ${ }^{2,5,6}$.

A positive Carnett's test result may also be found in the anterior cutaneous nerve entrapment syndrome (ACNES), a somewhat mysterious pain syndrome that is said to be caused by the trapping of end twigs of intercostal nerves at the level of the rectus abdominis muscle. This diagnosis is suggested by a combination of the patient's history (chronic pain), physical examination (circumscript pain localization and abnormal sensibility) and the absence of objective abnormalities (laboratory, ultrasound and/ or CT scan). If ACNES is considered, a local subfascial anesthetics injection (trigger point infiltration) may prove diagnostic and sometimes therapeutic ${ }^{7,8}$. Persistent pain relief is reported using sequential trigger point infiltrations combining anesthetics with corticosteroids, 9-13. If pain recurs, an anterior neurectomy can be beneficiary in recalcitrant patients. A cohort study recently reported on the results of a tailored regimen in 139 patients with suspected ACNES ${ }^{14}$. Half of these patients $(n=69)$ underwent a surgical neurectomy. At a median 18 months follow-up, $71 \%$ of the patients were still very satisfied with the clinical result of this procedure. However, randomized controlled data on surgery for ACNES are unavailable.

The aim of the present study was to evaluate the effect of an anterior neurectomy on pain perception in patients with suspected ACNES. Individuals undergoing a sham operation served as controls. We hypothesized that pain attenuation following neurectomy resulted from resection of the entrapped nerve at the level of the ventral rectus fascia and not from a placebo effect. 


\section{Materials and methods}

This randomized controlled trial was conducted at a large teaching hospital (Máxima Medical Center (MMC), Veldhoven) in the Southeastern part of the Netherlands. The MMC is an 865-bed community hospital serving a population of approximately 350,000 inhabitants. The Department of General Surgery has gained considerable experience in the treatment of chronic pain due to abdominal wall or groin nerve entrapment syndromes ${ }^{14-17}$. The hospital serves as a referral center for a substantial number of other Dutch hospitals including all 8 Academic Centers. The Medical Ethics Committee of MMC approved study design, protocol and informed consent procedures. The study was registered in the Dutch Clinical Trial Register (NTR2016). Design and reporting of this trial occurs according to the CONSORT guidelines ${ }^{18}$.

All patients $>18$ year suffering from locoregional abdominal pain for at least one month were eligible for study if the following criteria were met:

1. Unilateral single tender spot in the abdominal area (trigger point),

2. Constant site of abdominal tenderness with a small $\left(<2 \mathrm{~cm}^{2}\right.$, 'finger tip') area of maximal intensity situated within the lateral boundaries of the rectus abdominis muscle,

3. Tenderness increases by abdominal muscle tensing using the Carnett's test. During this test, the investigator localizes the point of maximal pain using his index finger. While maintaining the finger on this tender spot, the patient is asked to lift the upper torso or both legs. When pain intensity is increased by this maneuver, the origin of the pain is most likely located in the abdominal wall ${ }^{2,5}$, $6,19,20$,

4. Temporary pain response (> 50\% reduction on VAS) after a trigger point infiltration using an anesthetic agent,

5. Normal laboratory findings (C-reactive protein concentration $<6 \mathrm{mg} / \mathrm{L}$, serumleukocytes $4-10^{9} \mathrm{~L}$, urine sedimentation), and

6. No abnormal abdominal imaging (if previously performed)

7. Failed earlier conservative treatment

Exclusion criteria were surgical scar related pain syndromes, recent intra abdominal pathology, comorbidity or impaired communication. Patients that met these criteria were offered a repeat $10 \mathrm{cc} 1 \%$ Lidocaine injection combined with $40 \mathrm{mg}$ methylprednisolone. Only patients insufficiently responding to this injection regimen were considered for the present trial. All patients suffered from pain for at least 3 months prior to entering either surgical arm. If patients were eligible for study, informed consent was asked.

Study population characteristics were obtained including age, sex, length, weight and pain related specifics such as presumed etiology. Disability caused by the pain was measured on using a 4-point VRS. Participants were randomly assigned to one of the treatment groups following a computer-generated list of random numbers by blocks of eight. The allocation sequence was concealed from the researcher enrolling and assessing participants in sequentially numbered, opaque and sealed envelopes, prepared by a secretary with no involvement in the trial. Enrolment was performed in the outpatient department and patients were provided with substantial time to consider participation in the two to three weeks before surgery.

Figure 1 Intra-operative view of a neurovascular bundle (loop) and a nearby branch (loop) perforating the fascial foramina of the anterior sheath of the abdominal rectus muscle

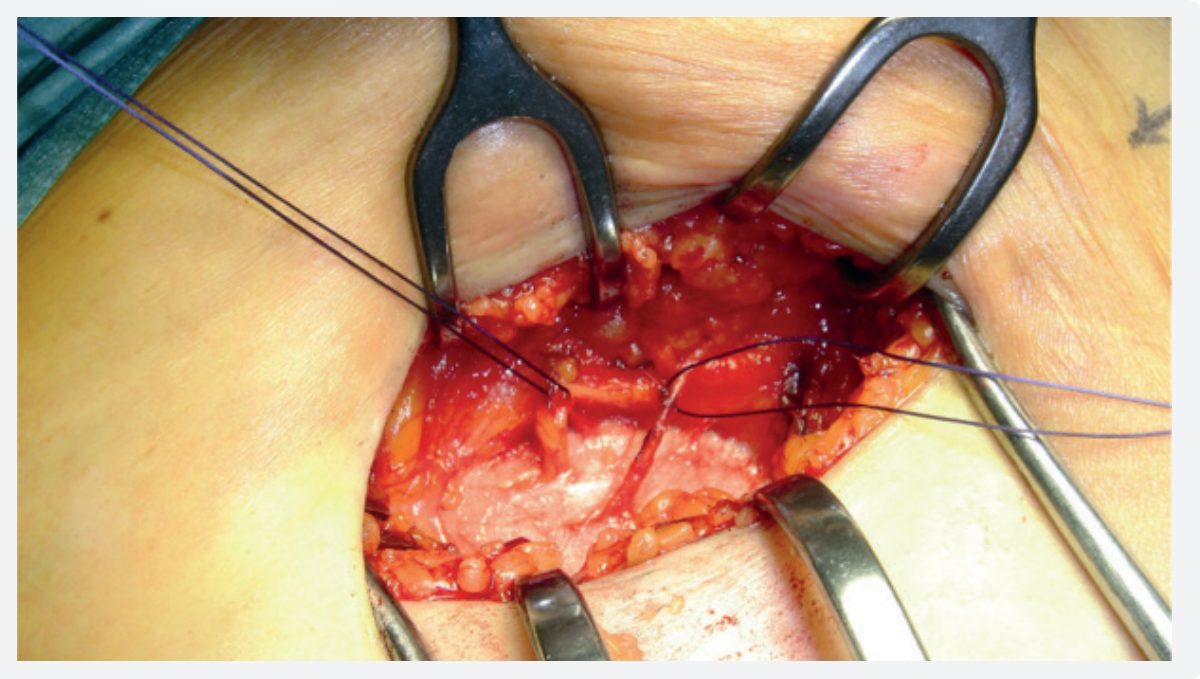

Patients were operated in a day care setting by one surgeon (RR). The area of maximal pain was identified using the Carnett's test and marked. Once general anesthesia was administered, a dedicated secretary was asked to open the next sequential envelope and to clearly state the allocated procedure (neurectomy or sham procedure). Name and 
date of birth of the participant were written on the envelope. The anterior sheath of the abdominal rectus muscle was exposed after skin and subcutaneous layer were opened via a 5-7 cm transverse incision. If a sham procedure was allocated, the procedure was terminated at this point and the wound was closed in layers. If a neurectomy was to be performed, neurovascular bundles penetrating the subcutaneous fat through pre-existent fascial foramina were identified. The fascia was opened and the bundle was ligated and removed as well as all its branches within a $5 \mathrm{~cm}$ radius (Figure 1). Accompanying vascular structures were ligated or coagulated. The sheath as well as the remainder of the wound was closed in layers using absorbable suturing material.

The wound of both study groups was infiltrated with $10 \mathrm{cc}$ of $0.25 \%$ bupivacaine. After discharge, patients were encouraged to resume daily activities as soon as possible.

The primary investigator $(\mathrm{OB})$ assessed outcomes in the outpatient department six weeks post operatively. Patients were interviewed and a physical examination was performed. The investigator and participants were blinded to the characteristics of the surgical procedure. Allocation was revealed only after completion of the 6-week study period or if an intercurrent event warranted re-intervention. If a sham procedure was performed with an unsuccessful outcome, patients were offered a reoperation including a real neurectomy within 6 weeks thereafter.

Endpoint was the proportion of patients achieving a minimal 50\% improvement in pain perception measured using a Visual Analogue Scale [VAS, $0 \mathrm{~mm}$ (pain absent) to $100 \mathrm{~mm}$ (excruciating pain)], and/or an at least 2-point point improvement on a Verbal Rating Scale (VRS 0-5, $0=$ no pain, $5=$ severe pain) at the 6 weeks time point postoperatively compared to the preoperative VAS and VRS

Based on the experience with a cohort of ACNES patients, the study was powered for the endpoint to detect a difference in proportion of successful response (minimal 50\% improvement in pain perception) of $75 \%$ in the neurectomy group versus a successful response of $30 \%$ in the sham surgery (placebo) group, with a two-sided 5\% significance level and a power of $80 \%{ }^{14}$. To achieve this, a sample size of 22 patients per group was required. A 24-month inclusion period was anticipated for recruitment. Since drop out or loss to follow up was not expected, trial sample size was maintained at this number of patients.

\section{Statistical analysis}

Baseline characteristics were presented as percentages, mean $( \pm S D)$ or median values (range) as appropriate. Differences in baseline characteristics between study groups were tested using a Chi square test for categorical variables and a Student's t-test (norma distribution) or Mann-Whitney test (skewed distribution) in case of continuous variables. The difference in proportions of success was calculated using the Yeates-corrected Ch Square. A p-value $<0.05$ was considered statistically significant. Data analysis was performed using SPSS 16 for Windows.

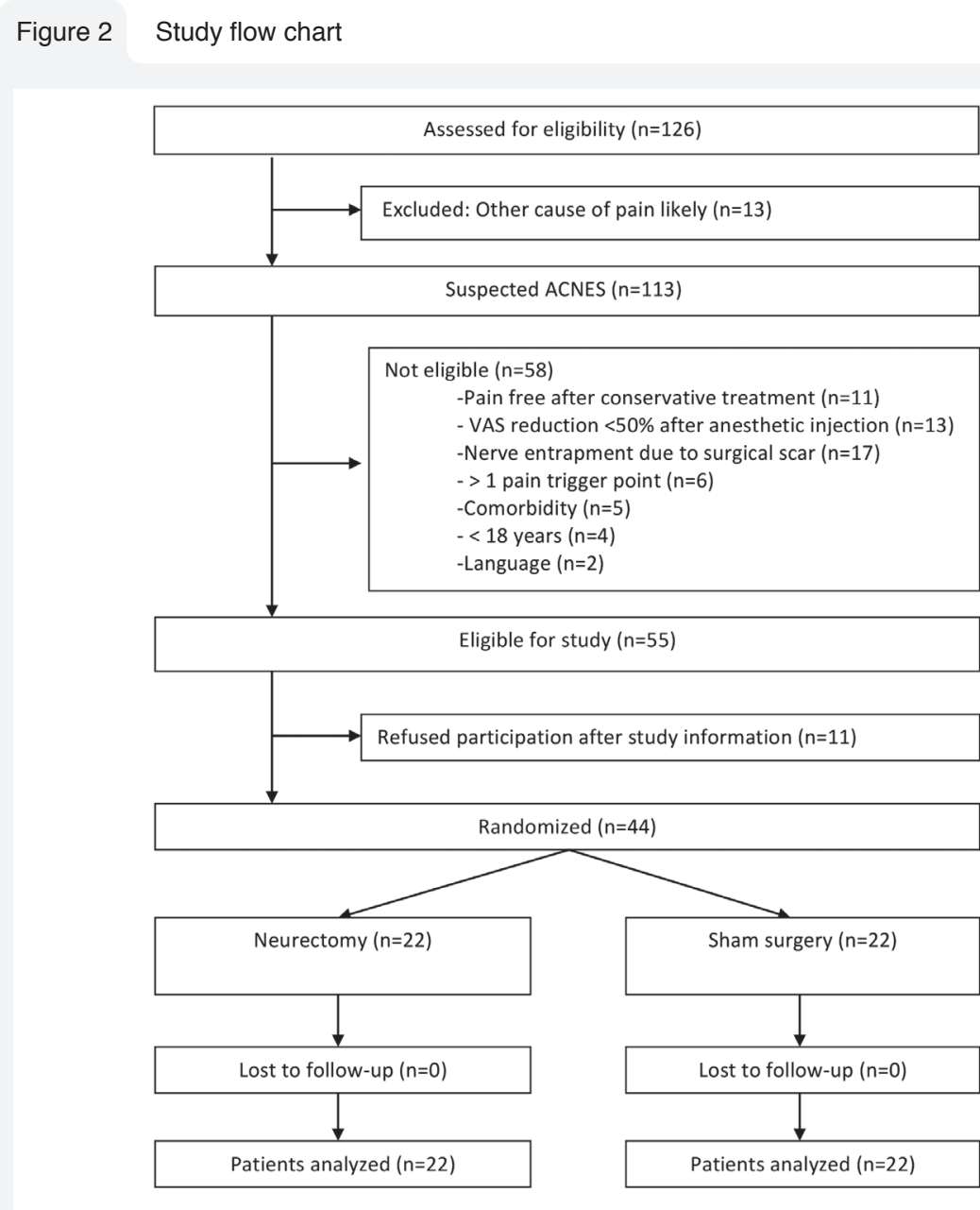




\section{Results}

Patients were recruited from August $1^{\text {st }} 2008$ through December $31^{\text {st }} 2010$. A total of 126 patients were evaluated for an alleged abdominal wall related pain (Figure 2). Based on physical examination and/or laboratory/imaging findings, the origin of the abdominal pain was considered less likely related to the abdominal wall in 13 patients. An additional group of 58 patients was excluded for reasons listed in figure 2. As 11 patients did not consent to participation, 44 patients were randomized after informed consent and all received the allocated intervention (neurectomy $n=22$; sham $n=22$ ). Baseline demographics, pain levels (VAS, VRS) and disability scores are summarized in table 1. Data accrual was complete without dropouts or loss to follow up. Individual patient data are presented in table 2 .

The number of patients demonstrating a successful response was significantly higher in the group receiving a neurectomy compared to sham surgery (neurectomy, $n=16 / 22$ versus sham, $n=4 / 22, p=0 \cdot 001$, Figure 3). Eighteen of the 22 sham patients reported an unsuccessful pain response and were all reoperated within 6 weeks after de-blinding. A reoperation resulted in 11 of these $18(61 \%)$ in successful pain reduction as again evaluated 6 weeks later. All 4 initially successful sham patients returned with pain within 6 months after surgery. A second procedure entailing a real neurectomy proved successful in 3 of these 4 .

Multiple penetrating nerve branches (median 2, range 1-3) were routinely found during the neurectomy procedure. Complications associated with surgery were localized hematoma ( $\mathrm{n}=5$, conservative treatment), infection ( $\mathrm{n}=1$, antibiotics and drainage) and increased level of local pain $(n=1)$.
Table 1 Baseline patient demographics, pain characteristics (VAS, VRS) and disability scores

\begin{tabular}{lcc} 
& Neurectomy (n=22) & Sham (n=22) \\
Age & $45(20-73)$ & $40(19-65)$ \\
Sex ratio $(\mathrm{M}: \mathrm{F})$ & $2: 20$ & $3: 19$ \\
Height $(\mathrm{cm})$ & $171(7) \mathrm{cm}$ & $168(5) \mathrm{cm}$ \\
Weight $(\mathrm{kg})$ & $76(21) \mathrm{kg}$ & $75(16) \mathrm{kg}$ \\
BMI $\left(\mathrm{kg} / \mathrm{m}^{2}\right)$ & $26(7)$ & $25(5)$ \\
\hline Etiology $(\mathrm{n})$ & & \\
Spontaneous & 15 & 17 \\
Sport & 1 & 1 \\
Job related & 0 & 0 \\
Recent abdominal & 4 & 3 \\
surgery & 0 & 0 \\
Pregnancy & 2 & 1 \\
Unusual activity & & \\
\hline Duration pain prior to surgery & & $12(3->60)$ \\
(Months) & $19(5->120)$ &
\end{tabular}
(Months)

17

19

around pain point
ara

17

$73(34-98)$

\section{VAS $(\mathrm{mm})$}

$0=$ No pain

$1=$ Very mild

$2=$ Mild

$3=$ Moderate

$4=$ Severe

$0 \quad 0$

Verbal Rating Scale Disability (n)

No pain on daily activities

Mild pain but no disability

Disabled during heavy activity

Disabled during light activity

Abdominal pain location

Right upper quadrant

Right lower

Left upper

0

3

9
10

0

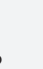

2
6

13

Left lower

\begin{tabular}{lc}
1 & 1 \\
8 & 5 \\
8 & 6 \\
5 & 10 \\
\hline
\end{tabular}

BMI = Body Mass Index

Although not contributing in the present cohort job related activities and pregnancy are considered etiologic factors. ${ }^{14}$

Means are presented with (SD) and medians with (Range) 
Table 1 Individual patient data, - indicates no reduction or even increase of pain

\begin{tabular}{|c|c|c|c|c|c|}
\hline $\begin{array}{l}\text { Randomization } \\
\text { number }\end{array}$ & $\begin{array}{l}\text { VAS Pre } \\
\text { Surgery }\end{array}$ & $\begin{array}{l}\text { VAS Post } \\
\text { Surgery }\end{array}$ & $\begin{array}{l}\text { Relative } \\
\text { reduction of } \\
\text { VAS }\end{array}$ & VRS reduction & $\begin{array}{l}\text { Succes by } \\
\text { definition }\end{array}$ \\
\hline \multicolumn{6}{|l|}{ Neurectomie } \\
\hline 3 & 60 & 18 & $70 \%$ & 2 & Yes \\
\hline 5 & 69 & 9 & $87 \%$ & 2 & Yes \\
\hline 6 & 85 & 0 & $100 \%$ & 3 & Yes \\
\hline 7 & 30 & 0 & $100 \%$ & 4 & Yes \\
\hline 9 & 50 & 0 & $100 \%$ & 3 & Yes \\
\hline 14 & 74 & 14 & $81 \%$ & 1 & Yes \\
\hline 15 & 35 & 44 & - & 0 & \\
\hline 16 & 34 & 35 & - & 0 & \\
\hline 17 & 22 & 4 & $82 \%$ & 2 & Yes \\
\hline 19 & 29 & 11 & $62 \%$ & 2 & Yes \\
\hline 22 & 76 & 0 & $100 \%$ & 3 & Yes \\
\hline 23 & 36 & 0 & $100 \%$ & 2 & Yes \\
\hline 25 & 67 & 5 & $93 \%$ & 3 & Yes \\
\hline 26 & 56 & 5 & $91 \%$ & 2 & Yes \\
\hline 27 & 53 & 5 & $91 \%$ & 3 & Yes \\
\hline 29 & 35 & 16 & $54 \%$ & 0 & Yes \\
\hline 30 & 62 & 80 & - & 0 & \\
\hline 35 & 31 & 38 & - & 0 & \\
\hline 38 & 45 & 53 & - & 0 & \\
\hline 40 & 76 & 78 & - & 0 & \\
\hline 42 & 52 & 15 & $71 \%$ & 2 & Yes \\
\hline 43 & 35 & 9 & $74 \%$ & 2 & Yes \\
\hline \multicolumn{6}{|l|}{ Sham } \\
\hline 1 & 12 & 83 & - & 0 & \\
\hline 2 & 34 & 42 & - & 0 & \\
\hline 4 & 29 & 27 & $7 \%$ & 1 & \\
\hline 8 & 72 & 45 & $37 \%$ & 0 & \\
\hline 10 & 94 & 83 & $12 \%$ & 0 & \\
\hline 11 & 68 & 4 & $94 \%$ & 3 & Yes \\
\hline 12 & 5 & 73 & - & 0 & \\
\hline 13 & 65 & 72 & - & 0 & \\
\hline 18 & 51 & 0 & $100 \%$ & 3 & Yes \\
\hline
\end{tabular}

\begin{tabular}{lllcll}
20 & 47 & 68 & - & 0 & \\
21 & 34 & 52 & - & 0 & \\
24 & 32 & 44 & - & 0 & \\
28 & 61 & 63 & - & 1 & \\
31 & 62 & 55 & $11 \%$ & 0 & Yes \\
32 & 38 & 10 & $74 \%$ & 1 & \\
33 & 47 & 42 & - & 0 & \\
34 & 52 & 47 & $11 \%$ & 0 & \\
36 & 24 & 51 & - & 0 & \\
37 & 71 & 10 & $86 \%$ & 2 & \\
39 & 55 & 81 & - & 0 & \\
41 & 63 & 68 & - & 0 & \\
44 & 58 & 38 & $44 \%$ & 1 & \\
\hline
\end{tabular}

VAS: Visual Analogue Scale, VRS: Verbal Rating Scale

Figure 3 Illustration of successful response (> 50\% VAS difference and/or 2 VRS categories) in two groups of patients with suspected ACNES, who either underwent a neurectomy or a sham procedure $(p=0.001)$; $Y$-axis shows the number of patients

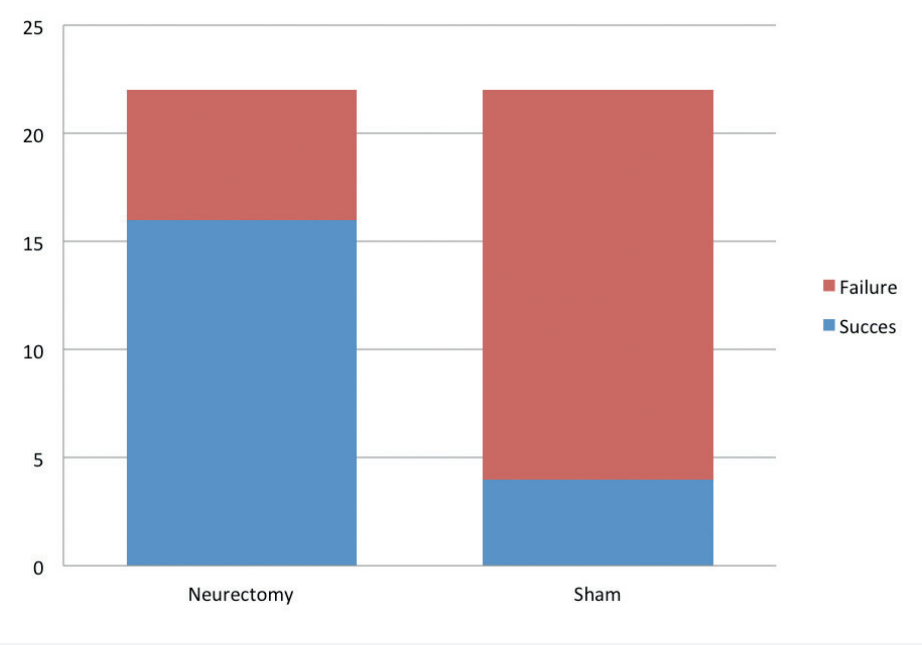




\section{Discussion}

The present trial is the first to demonstrate the efficacy of a neurectomy in patients with abdominal pain diagnosed with ACNES in a double blind, placebo controlled setting. Neurectomized individuals experienced significantly more often pain reduction compared to patients just undergoing a sham procedure. Since the number needed to treat is very low (treat 3 to cure 2), a reasonably limited surgical procedure such as an anterior neurectomy appears a very effective therapeutic option. Hitherto, the syndrome of ACNES is associated with skepticism that is fuelled by 'scientific' evidence provided by opinions of experts. For instance, a long lasting soothing effect after just one anesthetic injection is often reported in various abdominal wall pain syndromes including $\mathrm{ACNES}^{7,9-13}$. Since the exact patient composition of reported cohorts frequently remained unspecified, a positive or confirmative selection bias process likely influences this beneficial outcome. These subjective observations have led critics to suggest that a placebo phenomenon might be involved. However, a placebo controlled trial in alleged ACNES was considered difficult to ethically justify ${ }^{5}$. If pain associated with ACNES was refractory to all conservative stratagems, surgery was deemed acceptable as a final resort and sometimes appeared successful ${ }^{2}$. The present trial demonstrates that a placebo effect can be ignored as the major contributing factor to surgical success in ACNES. A neurectomy should therefore be considered as a treatment option in desperate patients not responding to conservative strategies.

Although considered the gold standard in contemporary research, one may question the ethics of randomized trials including sham surgery. However, a number of studies including a sham surgical arm performed under Institutional Review Board approved settings have delivered important and sometimes untoward results in the last 60 odd years. For instance, ligation of the internal mammary as a means to improve heart blood flow appeared completely ineffective as demonstrated in the late $1950{ }^{\prime 2} \mathrm{~s}^{21}$. A more recent blind, sham-controlled trial showed no benefit of most commonly performed arthroscopic procedures for osteoarthritis. ${ }^{22}$ As uterine nerves contain sensory afferents originating in the pelvic area, ablation was a routinely performed and generally accepted treatment in women with chronic pelvic pain and dysmenorrhea. However, a surgical trial with a sham arm provided evidence of just a limited effect of uterine nerve transection on dysmenorrhea in the absence of endometriosis. In contrast, nerve removal was ineffective in non-dysmenorrhoeic or endometriotic chronic pelvic pain ${ }^{23}$, 24. A novel invasive procedure requires an ongoing process of monitoring and evaluations prior to a large scale implementation. A double blind controlled trial including a sham surgery arm is currently the most powerful tool for a safe introduction of such novel procedures in surgical practice.

Findings of the present trial are in line with results of a recent study in ACNES patients demonstrating that 2 of 3 neurectomized patients are pain free after a 18 months follow up ${ }^{14}$. However, it is unclear why some patients fulfilling the strict set of 6 inclusion criteria did not benefit from surgery. In the present trial, a little over one fourth of the patients (6/22) receiving a neurectomy did not experience a significant pain reduction. A number of explanations may be forwarded. It is generally thought that nerve entrapment in ACNES is usually situated at the level of the superficial fascia of the rectus abdominis muscle. The injected anesthetic agent may have diffused towards more deeper located nerve portions leading to a successful pain response and subsequent study inclusion. As the neurectomy just included nerve end twigs piercing through ventral portions of the fascia, it may be that these surgical non responders have a type of ACNES characterized by nerve entrapment at dorsal or lateral portions of the muscle as hypothesized by others? This theory is also supported by the observation that some non-responders in a retrospective cohort did benefit from a second neurectomy at the dorsolateral side of the rectus muscle, at the level of the nerve entering the rectus sheet ${ }^{14}$. Failure to identify neighboring end branches responsible for the abdominal pain may also explain some of the non-responders. A median number of 2 nerve branches were found in the present trial. Routine exploration of a 5 to $5 \mathrm{~cm}$ area of the ventral fascia is advised as a means to reduce the percentage of surgical failures.

A small portion of the study patients $(n=4)$ appeared free of pain although nerve endings were not removed during the sham operation. Interestingly, although satisfied with their level of pain at the 6 weeks postoperative evaluation, all 4 opted for a reoperation in the ensuing months as 'the pain had recurred'. Three of these 4 reported a successful response after a true neurectomy. It is unclear why some patients experience beneficial effects of sham surgery. Some of the initial effects may be attributed to a placebo phenomenon. However, a plausible explanation may also be a long lasting anesthetic effect following the standard perioperative infiltration of the subfascial region and wound. Long-term pain reduction after local anesthetics in chronic abdominal pain syndromes is a commonly observed phenomenon. A lasting anesthetic effect far beyond the reported agent's half life may be due to a blockade of nerve transmission. Moreover, local anesthetics may also 
act upon sodium-channel isomers found on the nerve axons involved in chronic pain ${ }^{25}$. These findings support the necessary step of an injection scheme prior to embarking on more invasive treatment strategies for ACNES.

Although the syndrome of ACNES has occasionally received attention in the literature on chronic abdominal pain (CAP), a prominent place on the list of possible etiological factors of CAP was never attained. ACNES still remains a frequently overlooked entity and patients are subjected to prolonged doctor's delay ${ }^{2,4,12,26,27}$. The majority of patients in the present trial was referred to our clinic and also demonstrated a substantial median 13 months diagnostic delay (a few even as much as $>120$ months). It must be stressed that the diagnosis of ACNES is a typical old-fashioned 'clinical diagnosis'. It may be confidentially stated that patients harboring the triad of chronic abdominal pain, a circumscript pain point (in the presence of a positive Carnett's test and/or local sensory disturbances) and absence of abnormalities in blood analysis or US/CT suffer from ACNES until proven otherwise. The diagnostic algorithm is simple and can be readily employed by an array of health providers treating CAP patients. The surgical procedure should be restricted to recalcitrant patients but is safe with few complications and it can be executed in a day care setting. Such a simple diagnostic and therapeutic approach will indeed cut costs as the number of unnecessarily performed specialist consultations and visceral investigations will be reduced ${ }^{12,28}$. It is hoped that this study will contribute to an accelerated therapeutic pathway in patients with suspected ACNES.

We conclude that a ventral neurectomy is effective in a substantial part of patients with suspected ACNES failing to respond to a conservative treatment.

\section{References}

1. Gray DW, Dixon JM, Seabrook G, et al. Is abdominal wall tenderness a useful sign in the diagnosis of non-specific abdominal pain? Ann R Coll Surg Engl. 1988; 70: 233-234.

2. Lindsetmo RO, Stulberg J. Chronic abdominal wall pain -- a diagnostic challenge for the surgeon. Am J Surg. 2009; 198: 129-134.

3. McGarrity TJ, Peters DJ, Thompson C, et al. Outcome of patients with chronic abdominal pain referred to chronic pain clinic. Am J Gastroenterol. 2000; 95: 1812-1816.

4. Srinivasan R, Greenbaum DS. Chronic abdominal wall pain: a frequently overlooked problem. Practical approach to diagnosis and management. Am J Gastroenterol. 2002; 97 : $824-830$.

5. Abdominal wall tenderness test: could Carnett cut costs? Lancet. 1991; 337: 1134.

6. Carnett J. Intercostal neuralgia as a cause of abdominal pain and tenderness. Surg Gynecol Obstet. 1926: 625-632.

7. Applegate WV. Abdominal cutaneous nerve entrapment syndrome. Surgery. 1972; 71: $118-124$

8. Applegate WV, Buckwalter NR. Microanatomy of the structures contributing to abdominal cutaneous nerve entrapment syndrome. J Am Board Fam Pract. 1997; 10: 329-332.

9. Doouss TW, Boas RA. The abdominal cutaneous nerve entrapment syndrome N Z Med J. 1975; 81: 473-475.

10. Gallegos NC, Hobsley M. Abdominal wall pain: an alternative diagnosis. Br J Surg. 1990 77: 1167-1170.

11. Hall PN, Lee AP. Rectus nerve entrapment causing abdominal pain. Br J Surg. 1988; 75 917.

12. Hershfield NB. The abdominal wall. A frequently overlooked source of abdominal pain. J Clin Gastroenterol. 1992; 14: 199-202.

13. Slocumb JC. Chronic somatic, myofascial, and neurogenic abdominal pelvic pain. Clin Obstet Gynecol. 1990; 33: 145-153.

14. Boelens OB, Scheltinga MR, Houterman S, et al. Management of anterior cutaneous nerve entrapment syndrome in a cohort of 139 patients. Ann Surg. 2011; 254: 1054-1058.

15. Roumen RM, Scheltinga MR. [Abdominal intercostal neuralgia: a forgotten cause of abdominal pain]. Ned Tijdschr Geneeskd. 2006; 150: 1909-1915.

16. Loos MJ, Scheltinga MR, Roumen RM. Tailored neurectomy for treatment of postherniorrhaphy inguinal neuralgia. Surgery. 2010; 147: 275-281. 
17. Loos MJ, Scheltinga MR, Roumen RM. Surgical management of inguinal neuralgia after a low transverse Pfannenstiel incision. Ann Surg. 2008; 248: 880-885.

18. Schulz KF, Altman DG, Moher D. CONSORT 2010 Statement: updated guidelines for reporting parallel group randomised trials. Trials. 2010; 11: 32.

19. Thomson H, Francis DM. Abdominal-wall tenderness: A useful sign in the acute abdomen. Lancet. 1977; 2: 1053-1054.

20. Abdominal wall tenderness test. Lancet. 1991; 337: 1606-1607.

21. Cobb LA, Thomas GI, Dillard DH, et al. An evaluation of internal-mammary-artery ligation by a double-blind technic. N Engl J Med. 1959; 260: 1115-1118.

22. Moseley JB, O’Malley K, Petersen NJ, et al. A controlled trial of arthroscopic surgery for osteoarthritis of the knee. N Engl J Med. 2002; 347: 81-88.

23. Johnson NP, Farquhar CM, Crossley S, et al. A double-blind randomised controlled trial of laparoscopic uterine nerve ablation for women with chronic pelvic pain. Bjog. 2004; 111: 950-959.

24. Daniels J, Gray R, Hills RK, et al. Laparoscopic uterosacral nerve ablation for alleviating chronic pelvic pain: a randomized controlled trial. Jama. 2009; 302: 955-961.

25. Amir R, Argoff CE, Bennett GJ, et al. The role of sodium channels in chronic inflammatory and neuropathic pain. J Pain. 2006; 7: S1-29.

26. Marinacci AA, Courville CB. Radicular syndromes simulating intra-abdominal surgical conditions. Am Surg. 1962; 28: 59-63.

27. Sharpstone D, Colin-Jones DG. Chronic, non-visceral abdominal pain. Gut. 1994; 35: 833-836.

28. Thompson C, Goodman R, Rowe WA. Abdominal wall syndrome: a costly diagnosis of exclusion. Gastroenterol. 2001; 120: A637. 


\section{Chapter 7}

Long-term success rates after an anterior neurectomy in patients with an abdominal cutaneous nerve entrapment syndrome

Tijmen van Assen

Oliver B. Boelens

Percy V. van Eerten

Chrystel Perquin

Marc R. Scheltinga

Rudi M. Roumen 


\section{Abstract}

Background

Surgery is occasionally proposed in patients with chronic abdominal wall pain due to an anterior cutaneous nerve entrapment syndrome (ACNES) who are refractory to injection therapy. An anterior neurectomy may seem successful but follow-up is usually short and populations are small. Primary aim of this study was to determine the long-term success rate of surgery in a large ACNES population.

Methods

In this retrospective observational study, ACNES patients $\geq 18$ years who underwent a primary anterior neurectomy between January 2004 and February 2012 in one single center were studied. Pain scores were obtained before surgery, after surgery and at the moment of questioning using a pain intensity numeric rating scale (PINRS 0-10) and a 6-point verbal category rating scale (VRS). Success was defined as $\mathrm{a} \geq 50 \%$ PI-NRS reduction or $\geq 2$ point VRS reduction.

Results

Data of 181 neurectomies in 154 individuals were available for analysis (females, $\mathrm{n}=127,82.5 \%$; age $47 \pm 17$ years, range, $20-83$ ). Pain prior to surgery was severe (mean PI-NRS 8.08, SD 1.43). Short-term (1-3 months postoperative) success was $70 \%$ (127/181 procedures). Three subjects showed spontaneous remission of complaints after $\geq 3$ months. After a mean 32 months (range 3-93) follow-up, a success rate of $61 \%(109 / 180)$ on the long-term was found.

\section{Conclusion}

A $70 \%$ short-term success rate and a $61 \%$ long-term success rate following a primary anterior neurectomy in patients with chronic abdominal pain due to ACNES was attained. Surgery is the method of choice in ACNES patients refractory to a conservative regimen.

\section{Introduction}

Knowledge on the anterior cutaneous nerve entrapment syndrome (ACNES) as a neglected cause of chronic abdominal pain is limited ${ }^{1-13}$. ACNES patients experience severe neuropathic pain in the abdominal area that is caused by entrapped end twigs of intercostal nerves at the level of the anterior rectus sheath. Although abdominal pain is reported, they in fact suffer from pain originating in the abdominal wall. Patients harboring a triad of chronic abdominal pain, a circumscript pain point within the lateral boundaries of the rectus abdominis muscle (in the presence of a positive Carnett's test and/or local sensory disturbances), and the absence of abnormalities in blood analysis or ultrasonography/computed tomography are likely to suffer from ACNES until proven otherwise.

Two recent trials have shed light on diagnostic and therapeutic specifics regarding this syndrome. Pain reduction following trigger point infiltration using an anaesthetic agent in suspected ACNES patients was based on an anaesthetic mechanism but not on a placebo, dry needling, or a mechanical (volume) effect ${ }^{9}$. In a subsequent double blind randomized trial using a sham surgical arm, neurectomy of the intercostal nerve endings at the level of the anterior sheath of the abdominal rectus muscle ('anterior neurectomy') was effective for pain reduction in most patients failing to respond to a conservative regimen ${ }^{10}$.

Once recognized, ACNES patients with unacceptable pain levels are advised to undergo a treatment regimen starting with one (or more) local trigger point injections. This approach is long-term effective in one-thirds of the population. Surgery such as an anterior neurectomy may be discussed with refractory patients. This step-by-step treatment protocol leads to total or substantial pain relief in eighty percent of the ACNES population $^{8}$. Surgical success rates were encouraging on the short and median term. For instance, a $73 \%$ success rate was demonstrated 6 weeks postoperatively in 22 patients in a randomised controlled trial ${ }^{10}$. After 18 months of follow-up, a neurectomy was effective in $71 \%$ of 69 other individuals 8 . However, these populations were relatively small whereas the long-term efficacy of an anterior neurectomy is still a matter of uncertainty.

Neuroma formation with consequent recurrence of symptoms is a feared long-term sequela of any surgical procedure entailing a planned or accidental neurectomy $y^{14-17}$. The incidence of neuroma formation in neurectomized ACNES patients is unknown. Furthermore, a portion of initially 'cured' ACNES patients may experience recurrence of 
pain once the effects of the local anaesthetic (that is administered as standard procedure following the neurectomy) wears off. Aim of the present study is to determine the long-term success rate of an anterior neurectomy in a large ACNES population. It is hypothesized that the majority of initially successfully neurectomized patient remain free of abdominal pain on the long term.

\section{Materials ans methods}

\section{General information}

This retrospective observational study was performed between January 2011 and January 2013, analysing neurectomies performed between 2004 and 2012, in Máxima Medical Center (MMC), a 595-bed community hospital situated in the southern part of The Netherlands. A separate surgical outpatient department of MMC ('SolviMáx' Center of Excellence for Abdominal Wall and Groin Pain) has a keen interest in chronic abdominal wall pain and groin pain syndromes (such as inguinal neuralgias and Pfannenstiel nerve entrapments) ever since the beginning of the new millennium. An increasing number of patients are evaluated by a team of experts ${ }^{4,8-13,18-23}$. In the year of 2012, some 250 ACNES patients were treated in SolviMáx.

\section{Data collection}

Electronically stored data of all abdominal wall pain and groin pain patients receiving a neurectomy in MMC over an 8 year time period between January 2004 and February 2012 were retrospectively entered in a separate database. ACNES patients that were linked to registered operation codes were identified from this database. Only adult ACNES patients ( $\geq 18$ year at time of treatment) registered as having undergone a primary anterior neurectomy were eligible for study (figure 1). A primary anterior neurectomy was defined as the first attempt to remove portions of end twigs of one (or more) of the intercostal nerves surfacing at the level of the anterior rectus muscle sheath via an open surgical procedure.

Patients meeting inclusion criteria were studied using a standard approach. Individuals that were operated between 2004 and March 2011 were invited to respond to a questionnaire containing a set of questions that was sent by mail between March-May, 2011. Non-responders and patients operated between March 2011 and February 2012
Figure 1 Study flowchart. Short and long term success following an anterior neurectomy for ACNES. Numbers between parentheses represent procedures

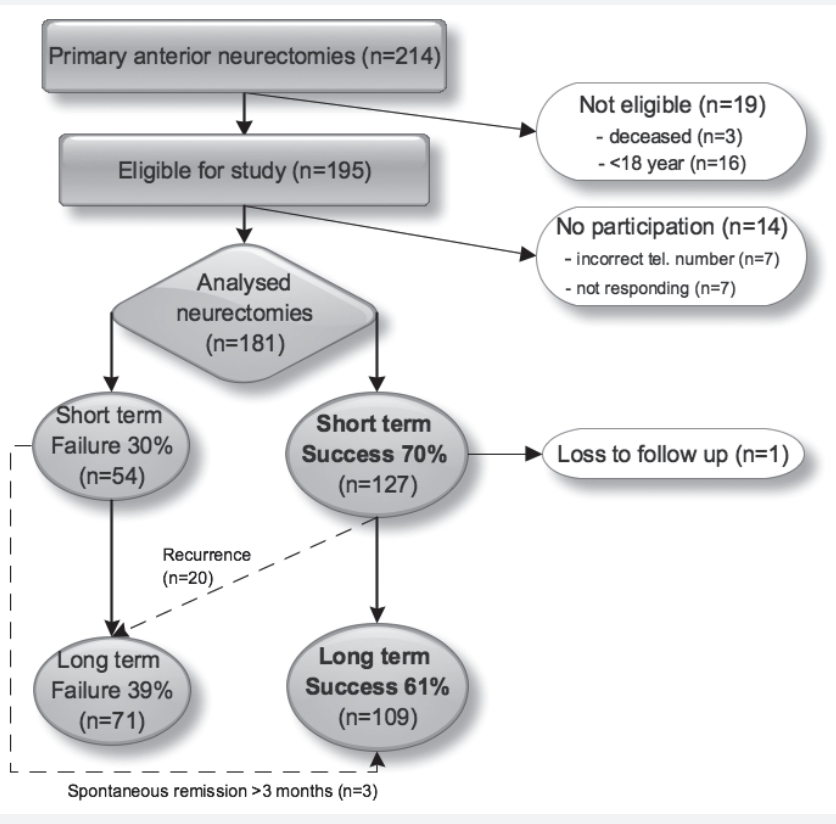

were additionally interviewed by phone by the first author using the same set of questions between July 2012 and February 2013

\section{Surgical specifics of an anterior neurectomy}

Patients are operated in same day surgery as previously reported ${ }^{8,10}$. The 'area of interest' supposedly containing the nerves responsible for the pain is marked preoperatively on the basis of a combination of a successful response on previous trigger point infiltration, currently reported pain using palpation, Carnett's test and altered skin sensibility ${ }^{24-26}$ After induction of general anaesthesia, the anterior sheath of the abdominal rectus muscle is exposed via a transverse $5-7 \mathrm{~cm}$ skin incision. The neurovascular bundle penetrating fascial foramina underneath the marked skin area is identified. The foramen is widened and the neurovascular bundle is removed over a $1-3 \mathrm{~cm}$ length. The proximal portion of the bundle is allowed to retract deeply into the rectus muscle. Additional nerve branches penetrating the rectus sheath within a $5-\mathrm{cm}$ radius are also removed. Accompanying small vessels are ligated or coagulated. Widened fascial foramina are 
closed using a running absorbable suture followed by a standard closing technique of the remainder of the incision. The surgical area is infiltrated with $10 \mathrm{ml}$ of $0.25 \%$ bupivacaine at the end of the procedure. A surgical procedure usually lasts between 30-60 minutes. Postoperative wound pain management included the continuation of paracetamol, nonsteroidal anti-inflammatory drugs or opiate analgesic drugs as also administered in the preoperative phase, or if deemed necessary. Patients were checked at least twice during a 6 week postoperative time period, usually via a visit on the outpatient department and via a telephone consultation. Specifics and complications were recorded into the hospital's electronic patient file.

\section{Short and long term follow-up}

All study patients were retrospectively asked to report pain in the scar area as experienced in the immediate preoperative phase, pain levels at the postoperative 1-3 months time point (short term follow-up), and pain at present or at the moment of recurrent abdominal pain $\geq 3$ months postoperative (long term follow-up). Both a 0-10 pain intensity numeric rating scale (PI-NRS) and a 6-point verbal categorical rating scale (VRS) (Table 1) were used $^{27-30}$

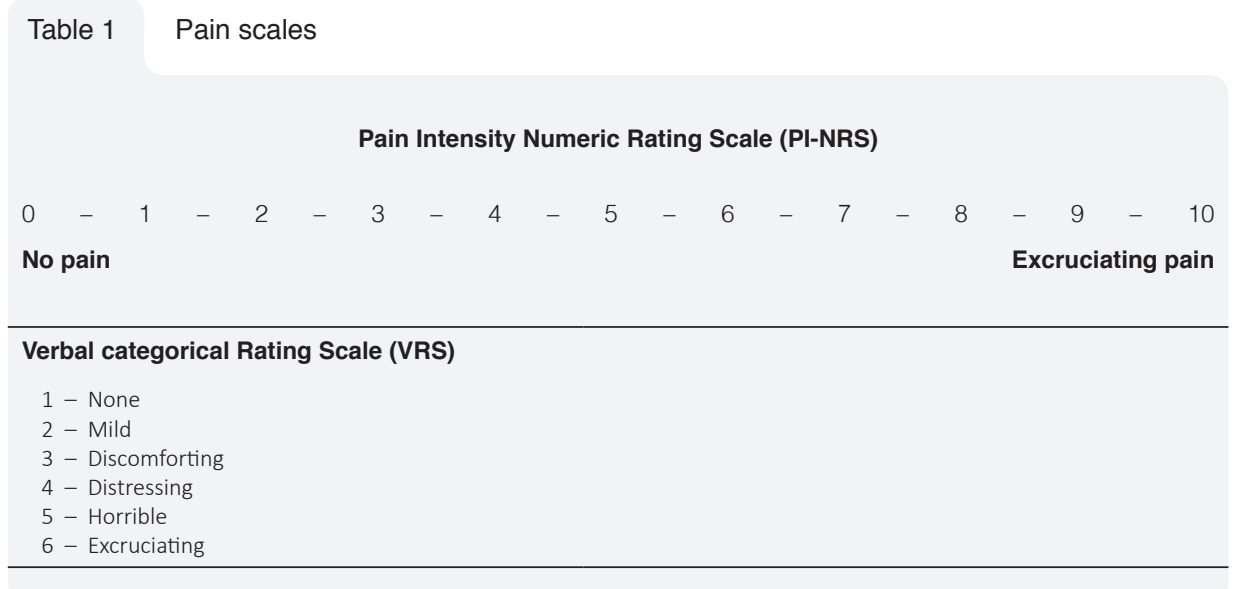

Short-term surgical success was defined as a $\geq 50 \%$ pain reduction using PI-NRS and/ or a minimal 2-point reduction using VRS at the postoperative 1-3 months time point, as suggested in previous studies ${ }^{8-10}$. All other scores were considered failures. For short-term failures who did not demonstrate pain reduction on the long-term, this time point was considered the endpoint of the study.

Long-term success was attained if present pain scores were $\leq 50 \%$ of preoperative pain levels using PI-NRS or if the present VRS was at least 2-point lower. Conversely, data of short-term successful patients with recurrent pain (scores $>50 \%$ of preoperative PI-NRS and $<2$-point lower VRS) that developed in proximity to the surgical scar at any momen between the primary anterior neurectomy and the long-term follow-up evaluation were studied in detail by three authors separately (TvA, RR, MS). If disagreement was initially present between these three, the decision on cause for long-term pain was eventually based on consensus.

Four types of abdominal pain on the long-term after an initial pain free period were distinguished:

1. 'Recurrent disease' was defined as the situation that an ACNES patient developed recurrent neuropathic pain in/underneath the scar area after a pain free period of $\geq 3$ months following the primary anterior neurectomy. The surgical result was interpreted as long-term failure.

2. 'Residual disease' was defined as recurrence of (for the patient recognizable) neuropathic pain $<3$ months after the primary anterior neurectomy. The initial postoperative pain reduction was considered due the effect of the local anaesthetic being a standard element of the surgical procedure. These cases were interpreted as short-term surgical failures.

3. 'De novo ACNES': symptoms and signs of ACNES in an area at least $5 \mathrm{~cm}$ distant to the neurectomy scar, suggesting entrapment of a neighbouring intercostal nerve or a new ACNES on the contralateral side. However, pain was not experienced in the area of the primary anterior neurectomy. Therefore, the surgical result was interpreted as long-term successful.

4. 'Abdominal pain other than ACNES'. Although subjects experience pain, this pain was not related to ACNES. The surgical result was interpreted as long-term successful.

Calculation of short and long-term success rates was made after patients with pain on the long-term after an initial pain free period were categorised. 
Recall bias

As a form of recall bias was feared in this retrospective study design, pre and postoperative values as recorded in the electronic patient files (if available) were compared with recalled values as reported in the questionnaire. As pain scores were not registered routinely in all electronic patient files, prospectively registered pain scores were exclusively used to investigate the presence of recall bias. Conversely, only retrospectively obtained pain scores were used for the analysis of treatment success.

Data analysis

Data analysis was performed using SPSS 20.0 for Mac OS X. Changes in PI-NRS and VRS over time were compared individually to determine the operative success. Descriptive PI-NRS and VRS measures were presented as percentages, mean values $( \pm \mathrm{SD})$ or median values (range) as appropriate. Differences in PI-NRS and VRS on various moments were calculated using a paired sample T-test (normal distribution) or Wilcoxon signed rank test (skewed distribution). Pearson correlation coefficients were calculated to determine the strength of linear dependence between the two variables.

\section{Results}

Study population

Between January 2004 and February 2012, 626 subjects with abdominal wall or groin pain underwent 806 neurectomies. Of these, a total of 214 primary anterior neurectomies were performed in 186 subjects with ACNES. As patients $<18$ year $(n=16)$ were excluded and 3 patients had deceased, 195 procedures in 167 subjects were eligible for analysis. Questionnaire data from 93\% procedures in 154 subjects were analysed (181/195, nonresponding $n=7$, no contact address $n=7$ ).

Most patients were female ( $\mathrm{n}=127,82.5 \%)$. The median age was $47 \pm 17$ years (range, 20-83). Some 23 patients were operated twice for ACNES that was present in two different abdominal areas, whereas two subjects received surgery three times for ACNES originating in three different abdominal areas. Fifteen patients reported minor complications after the procedure (8\%). A hematoma was conservatively managed in 10 . One hematoma, one seroma and three wound abscesses needed surgical drainage in the remaining five patients (table 2).
Table 2 Characteristics of surgical procedure

\begin{tabular}{l|c} 
Primary anterior neurectomies, $\mathrm{n}$ & 181 \\
\hline Location of surgery, quadrant, $\mathrm{n}(\%)$ & \\
Right upper & $22(12)$ \\
Left upper & $18(10)$ \\
Right lower & $93(51)$ \\
Left lower & $40(22)$ \\
Bilateral & $8(5)$ \\
\hline Immediate postoperative complications, $\mathrm{n}(\%)$ & $15(8)$ \\
Hematoma (conservative treatment) & 10 \\
Hematoma (surgical treatment) & 1 \\
Seroma (surgical) & 1 \\
Abscess (surgical) & 3 \\
\hline
\end{tabular}

\section{Short-term and long term results}

At the 1-3 months postoperative evaluation, some 132 procedures were by definition successful. Interestingly, 3 initially unsuccessful subjects became pain free in the $\geq 3$ months postoperative period. After a mean 32 months follow-up (range, 3-93; loss to follow-up, $\mathrm{n}=1$ ), success was attained in 101 procedures, indicating (some degree of) pain had recurred after an initial pain free period in 33 primary anterior neurectomies. Thes 33 primary anterior neurectomies were performed in 33 individuals.

Fate of patients $(n=33)$ with long-term pain after an initial pain free period

Recurrent disease was diagnosed in 20 of these 33 patients following a mean 10-month period of success (range 3-48). A re-exploration in 15 of these 20 patients revealed a (histologically proven) traumatic neuroma in three. A wait and see policy was followed in the remaining 5 subjects.

'Residual disease' was diagnosed in 5 patients, whereas 'de novo ACNES' was diagnosed in 6 additional patients ( 3 lower level, 1 higher level, 2 contralateral). Abdominal pain other than ACNES was diagnosed in two remaining patients.

\section{Calculation of short-term and long-term success}

Short-term success of the primary anterior neurectomy was $70 \%(n=127 / 181)$. The longterm success of the operation was $61 \%(n=109 / 181)$. Long-term failures $(n=71)$ included 
51 short-term failures and 20 recurrences. A $16 \%$ ( $n=20 / 127$, figure 1) recurrence rate was thus calculated.

Levels of pain are depicted in figure 2. Interestingly, the short-term failure group $(n=51)$ demonstrated postoperative scores that were significantly lower compared to preoperative level (postoperative 6.55 SD 2.10 vs. preoperative 7.67, SD 1.41; $\mathrm{p}=0.001$ ). In the subgroup with recurrent disease $(n=20)$, long-term pain levels were not different compared to preoperative levels (postoperative 8.15 , SD 1.56 vs. preoperative $8.60, \mathrm{SD}$ $1.35 ; \mathrm{p}=0.10)$.

Figure 2 Pain reduction in 3 ACNES populations. In the short-term failure group the short-term postoperative measurement was the end-point of the study. (*statistically significant; ns not significant, compared to pre-operative)

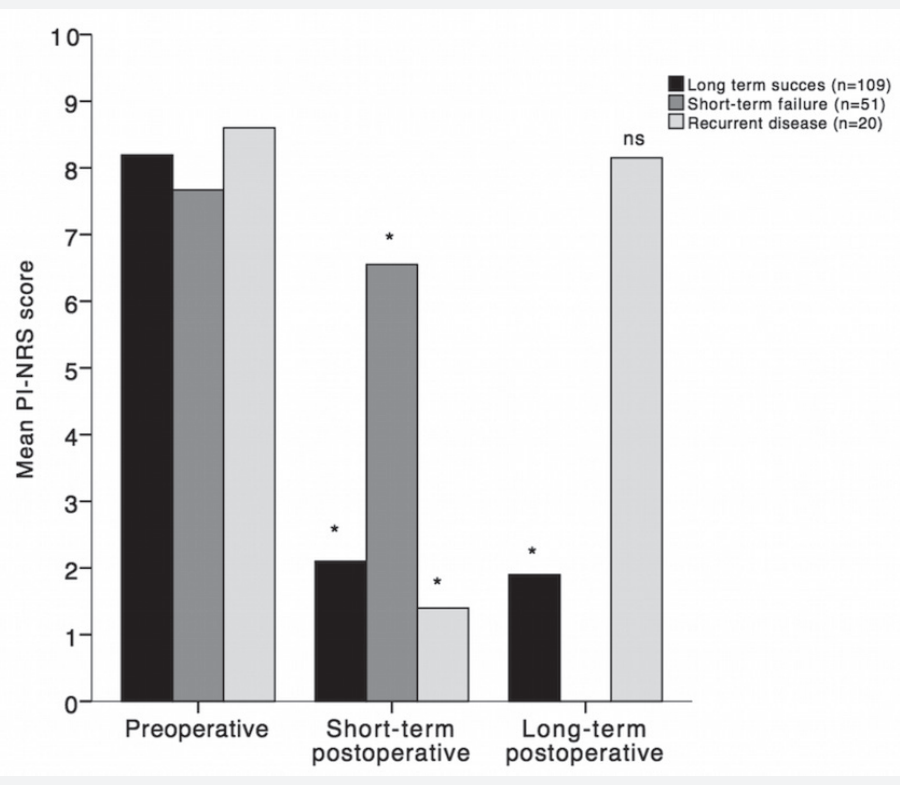

Recall bias

Pain scores (PI-NRS) that were prospectively obtained in the preoperative situation were obtained in half of the procedures $(49 \%, 88 / 181)$. These values were consistently lower compared to retrospectively obtained values (prospective: mean 7.69, SD 1.22, range 5-10 vs. retrospective: $8.28 \mathrm{SD} 1.34$, range $2-10 ; \mathrm{p}<0.001$ ). In addition, prospectively obtained postoperative values $(43 \%, 77 / 181)$ were also lower compared to retrospectively obtained pain (prospective: mean 2.45, SD 2.97, range $0-10$ vs. retrospective: 3.26 , SD 3.00 range $0-9 ; \mathrm{p}<0.001)$. These results indicate the presence of a structural but relative discrete recall bias effect, although it is appreciated that data were available in just half of the population. A high Pearson correlation coefficient was found between the two variables $(\rho=0.91)$. No difference was found regarding this effect when subgroup analysis was performed on either the successful or the failure group (data not shown).

\section{Discussion}

The present study in a large group of ACNES patients confirms a $70 \%$ shortterm surgical success rate of a primary anterior neurectomy as also found in smaller populations $s^{8,10}$. The $61 \%$ long-term success rate with a mean 32 months follow-up period is acceptable. The $16 \%$ recurrence rate is a more reliable estimation compared to a $4 \%$ rate as reported previously in a smaller group with a shorter follow-up ${ }^{8}$. Patients should both be counselled on the $70 \%$ success rate on the short-term as on the substantial recurrence rate associated with the primary anterior neurectomy procedure on the long-term.

One study recently proposed a standard step-by-step treatment strategy for ACNES. An anterior neurectomy is the alternative invasive option once injection therapy fails to confer success. This situation is real in 2 of 3 ACNES patients as approximately $30 \%$ remain permanently pain free after injection therapy only ${ }^{8}$. How should a $61 \%$ long-term success rate of a primary anterior neurectomy be judged? Such data should be viewed in the light of published 30-40\% short-term success rates of pharmacological treatment strategies for chronic peripheral neuropathic pain ${ }^{31,32}$. Unfortunately, most pharmacological treatments harbour unfavourable side effects whereas long-term efficacy is frequently not reported in these studies but is likely lower ${ }^{31-33}$. Evidence regarding alternative treatment options such as peripheral stimulation or deep brain stimulation for these pain syndromes is scarce $^{34}$. On the contrary, an anterior neurectomy is a simple procedure with few minor complications (table 2). Confirming the conclusion of two relative small other reports, data of the here presented largest study to date $(n=181)$ strongly supports the contention that a primary anterior neurectomy currently is the treatment of choice in ACNES patients that are refractory to the first step injection regimen ${ }^{8,10}$

Sole aim of the present investigation was to study the outcome of the primary anterior neurectomy. In our SolviMáx Center of Excellence, knowledge on alternative treatment 
options after recurrent or residual pain following an anterior neurectomy is building. Although high-level evidence is currently lacking, some of our patients benefitted from additional surgical explorations. Fifteen of the 20 recurrent pain patients underwent a second operation. In 3 of these, a histologically proven neuroma was found. Other patients with persistent pain were referred to alternative pain specialists. We are currently involved in studying the fate of these patients. However, it is essential that a number of recalcitrant patients require a multidisciplinary specialist team capable of offering multimodal treatment options.

An interesting observation is a significantly lower postoperative pain score in the 'failure group'. Although the neurectomy was a failure according to our strict definition (failure: current pain scores $>50 \%$ by PI-NRS or $<2$-points attenuation by VRS compared to preoperatively), these patients experienced an attenuated pain level after the operation. This phenomenon may be related to mechanisms of recognition and confirmation. Merited attention for their problem may contribute to coping leading to acceptance and subsequently a lower pain perception.

This study potentially harbours flaws associated with its retrospective character. Due to recall bias, pain perception may change over time. As a result, retrospectively obtained PI-NRS and VRS scores possibly do not correctly reflect pain levels as experienced in the past. However, our results comparing retrospectively and prospectively collected data demonstrated a minimal difference in pain intensity over time (preoperative difference 0.59 on a $0-10$ point scale, postoperative 0.81 ). For the purpose of this study, the influence of recall bias was therefore neglected since just considerable pain reduction ( $\geq 50 \%$ PINRS reduction and/or $\geq 2$ point reduction on VRS) was relevant for the primary study aim. It was initially thought that, depending on the treatment result (success or failure), perception of pain would differ over time. However, results of the present study do not support this contention.

We strongly feel that ACNES is still an overlooked entity although its existence is nowadays undisputed ${ }^{8-10,12}$. In current practice with nearly unlimited diagnostic options, the need for proper physical examination in chronic pain patients may seem of minor importance. However, abdominal wall pain syndromes including ACNES are typical clinical entities that are diagnosed with the doctor's ears and fingers. Patients reporting chronic abdominal pain with a circumscript pain point (trigger point) within the lateral boundaries of the rectus abdominis muscle (in the presence of a positive Carnett's test and/ or local somatosensory disturbances surrounding the pain point) having normal blood results and no imaging abnormalities likely suffer from an abdominal wall syndrome such as ACNES. With Carnett's test, the trigger point is localized and palpated using a finger. While the finger remains positioned onto the painful spot, tenderness increases when abdominal muscles are tensed as the patient lifts his/her head. Skin sensibility is tested by the use of a simple swab. Either a normal skin, hypoesthesia, hyperesthesia and sometimes even allodynia may be diagnosed. A cold alcohol gauze can also be used to test the vital sensibility. These simple tests greatly aid in arriving at a proper diagnosis of a neuropathic pain syndrome such as ACNES.

Pain specialists have long thought that 'cutting nerves is just not done'. It is said that this type of surgery invariably leads to either post-dissection pain, de-afferentation pain or phantom pain. These pain syndromes are only amenable to medication with centrally working mechanisms. However, these concepts are challenged ${ }^{35}$. Also neuroma formation with consequent pain recurrence (occasionally more severe than ever) is a feared long-term result of any surgical procedure entailing a neurectomy ${ }^{14-17}$. The present analysis shows that these fears may not be true as levels of pain in these recurrences were comparable (and not worse) to preoperative values. On the other hand, short and long-term pain relief after a primary anterior neurectomy is far superior compared to any other treatment strategy for this type of severe neuropathic pain. Therefore, we propose to adhere to the current step-by-step treatment protocol (injections followed by surgery in non-responding patients) in future ACNES patients. This complete regimen of consecutive local trigger point injections followed by a primary anterior neurectomy in recalcitrant ACNES patients is long-term effective in about three-quarters of the cases. However, some patients stand a small chance of developing a new ACNES pain syndrome in another abdominal area as was demonstrated in this study.

\section{Conclusion}

A $70 \%$ short-term success rate and a $61 \%$ long-term success rate following a primary anterior neurectomy is reported in a large population of patients with anterior cutaneous nerve entrapment syndrome. Surgery is the method of choice in ACNES patients who are refractory to conservative pain treatment strategies. 


\section{References}

1. Applegate WV. Abdominal Cutaneous Nerve Entrapment Syndrome (ACNES): A Commonly Overlooked Cause of Abdominal Pain. Perm J. 2002; 6: 20-27.

2. Hershfield NB. The abdominal wall. A frequently overlooked source of abdominal pain. J Clin Gastroenterol. 1992; 14: 199-202.

3. Ranger I, Mehta M, Pennington M. Abdominal wall pain due to nerve entrapment. Practitioner. 1971; 206: 791-792.

4. Roumen RM, Scheltinga MR. [Abdominal intercostal neuralgia: a forgotten cause of abdominal pain]. Ned Tijdschr Geneeskd. 2006; 150: 1909-1915.

5. Sharpstone D, Colin-Jones DG. Chronic, non-visceral abdominal pain. Gut. 1994; 35: 833-836.

6. Srinivasan R, Greenbaum DS. Chronic abdominal wall pain: a frequently overlooked problem. Practical approach to diagnosis and management. Am J Gastroenterol. 2002; 97: 824-830.

7. Suleiman S, Johnston DE. The abdominal wall: an overlooked source of pain. Am Fam Physician. 2001; 64: 431-438.

8. Boelens OB, Scheltinga MR, Houterman S, et al. Management of anterior cutaneous nerve entrapment syndrome in a cohort of 139 patients. Ann Surg. 2011; 254: 1054-1058.

9. Boelens OB, Scheltinga MR, Houterman S, et al. Randomized clinical trial of trigger point infiltration with lidocaine to diagnose anterior cutaneous nerve entrapment syndrome. Br J Surg. 2013; 100: 217-221.

10. Boelens OB, van Assen T, Houterman S, et al. A double-blind, randomized, controlled trial on surgery for chronic abdominal pain due to anterior cutaneous nerve entrapment syndrome. Ann Surg. 2013; 257: 845-849.

11. Scheltinga MR, Boelens OB, Tjon ATWE, et al. Surgery for refractory anterior cutaneous nerve entrapment syndrome (ACNES) in children. J Pediatr Surg. 2011; 46: 699-703.

12. van Assen T, Boelens OB, Kamphuis JT, et al. Construction and validation of a questionnaire distinguishing a chronic abdominal wall pain syndrome from irritable bowel syndrome. Frontline gastroenterol. 2012; 3: 288-294.

13. van Assen T, de Jager-Kievit JW, Scheltinga MR, et al. Chronic abdominal wall pain misdiagnosed as functional abdominal pain. J Am Board Fam Med. 2013; 26: 738-744.

14. Ducic I, Moxley M, Al-Attar A. Algorithm for treatment of postoperative incisional groin pain after cesarean delivery or hysterectomy. Obstet Gynecol. 2006; 108: 27-31.
15. Ducic I, Dellon AL. Testicular pain after inguinal hernia repair: an approach to resection of the genital branch of genitofemoral nerve. J Am Coll Surg. 2004; 198: 181-184.

16. Starling JR, Harms BA, Schroeder ME, et al. Diagnosis and treatment of genitofemoral and ilioinguinal entrapment neuralgia. Surgery. 1987; 102: 581-586.

17. Lee CH, Dellon AL. Surgical management of groin pain of neural origin. J Am Coll Surg. 2000; 191: 137-142.

18. Loos MJ, Roumen RM, Scheltinga MR. Chronic sequelae of common elective groin hernia repair. Hernia. 2007; 11: 169-173

19. Loos MJ, Roumen RM, Scheltinga MR. Classifying post-herniorrhaphy pain syndromes following elective inguinal hernia repair. World J Surg. 2007; 31: 1760-1765; discussion 1766-1767.

20. Loos MJ, Scheltinga MR, Mulders LG, et al. The Pfannenstiel incision as a source of chronic pain. Obstet Gynecol. 2008; 111: 839-846.

21. Loos MJ, Scheltinga MR, Roumen RM. Surgical management of inguinal neuralgia after a low transverse Pfannenstiel incision. Ann Surg. 2008; 248: 880-885.

22. Loos MJ, Scheltinga MR, Roumen RM. Tailored neurectomy for treatment of postherniorrhaphy inguinal neuralgia. Surgery. 2010; 147: 275-281.

23. Loos MJ, Verhagen T, Scheltinga MR, et al. A randomised controlled trial of injection therapy versus neurectomy for post-herniorrhaphy inguinal neuralgia: rationale and study design. Hernia. 2010; 14: 593-597.

24. Abdominal wall tenderness test: could Carnett cut costs? Lancet. 1991; 337: 1134

25. Thomson H, Francis DM. Abdominal-wall tenderness: A useful sign in the acute abdomen. Lancet. 1977; 2: 1053-1054.

26. Gallegos NC, Hobsley M. Recognition and treatment of abdominal wall pain. J R Soc Med. 1989; 82: 343-344.

27. Farrar JT, Young JP, Jr., LaMoreaux L, et al. Clinical importance of changes in chronic pain intensity measured on an 11-point numerical pain rating scale. Pain. 2001; 94: 149-158.

28. Moulin DE, Hagen N, Feasby TE, et al. Pain in Guillain-Barre syndrome. Neurology. 1997; 48: 328-331.

29. Aicher B, Peil H, Peil B, et al. Pain measurement: Visual Analogue Scale (VAS) and Verbal Rating Scale (VRS) in clinical trials with OTC analgesics in headache. Cephalalgia. 2011; 32: 185-197.

30. Breivik H, Borchgrevink PC, Allen SM, et al. Assessment of pain. Br J Anaesth. 2008; 101: 17-24. 
31. Finnerup NB, Otto M, McQuay HJ, et al. Algorithm for neuropathic pain treatment: an evidence based proposal. Pain. 2005; 118: 289-305.

32. Attal N, Cruccu G, Baron R, et al. EFNS guidelines on the pharmacological treatment of neuropathic pain: 2010 revision. Eur J Neurol. 2010; 17: 1113-e1188.

33. Freynhagen R, Bennett MI. Diagnosis and management of neuropathic pain. Br Med J. 2009; 339: b3002.

34. Cruccu G, Aziz TZ, Garcia-Larrea L, et al. EFNS guidelines on neurostimulation therapy for neuropathic pain. Eur J Neurol. 2007; 14: 952-970.

35. Lyftogt J. Pain Conundrums: Which Hypothesis? Central nervous system sensitization versus peripheral nervous system autonomy. Aust Musculoskeletal Med. 2008; 13: 72-74. 


\section{Chapter 8}

Surgical options after a failed neurectomy in anterior cutaneous nerve entrapment syndrome

Tijmen van Assen

Oliver B. Boelens

Percy V. van Eerten

Marc R. Scheltinga

Rudi M. Roumen 


\section{Abstract}

Background

Injection treatment followed by an anterior neurectomy in patients insufficiently responding to an injection regimen is successful long term in three-quarters of patients with anterior cutaneous nerve entrapment syndrome (ACNES). The efficacy of secondary surgery, including re-exploration or a posterior neurectomy in patients reporting recurrent pain after initially successful surgery or following an immediately failed anterior neurectomy is unknown.

\section{Methods}

A database of ACNES patients receiving surgery between 2004 and 2012 in the SolviMáx institution was analysed. Adult patients with residual pain after an anterior neurectomy (failures) or with recurrent pain after initially successful surgery (recurrences) were selected. Following a re-exploration or a posterior neurectomy, pain was scored using a pain intensity numeric rating scale (PI-NRS 0-10) and a six-point verbal category rating scale (VRS). Success was defined as a $\geq 50 \%$ PI-NRS reduction and/or $\geq 2$ point VRS reduction.

\section{Results}

ACNES patients undergoing an anterior neurectomy $(\mathrm{n}=181)$ were analysed during the 8-year study period. At follow up, 51 patients reported unacceptable pain levels following an anterior neurectomy, whereas 20 developed recurrent abdominal wall pain. Of these 71 unsuccessful patients, 41 underwent secondary surgery including a re-exploration $(n=10)$ or a posterior neurectomy $(n=31)$. After a 25 months median follow-up, secondary surgical treatment regimens proved successful in 66\% (27/41). Patients with recurrent pain did better (14/15) than patients who were immediate failures after the anterior neurectomy $(13 / 26, \mathrm{p}=0.01)$.

Conclusion

Secondary surgery including re-explorations and posterior neurectomies are successful in two-thirds of ACNES patients with persistent pain or recurrence of pain after an anterior neurectomy.

\section{Introduction}

The anterior cutaneous nerve entrapment syndrome (ACNES) is a condition that is caused by entrapped intercostal nerves leading to local abdominal pain that is located within the lateral boundaries of the abdominal rectus muscle. ACNES is an universally neglected cause of chronic neuropathic abdominal pain. Several treatment options have been forwarded in recent years ${ }^{1-6}$. A regimen of consecutive local trigger point injections is long-term effective in one third of patients with $\mathrm{ACNES}^{1}$. In patients failing such a conservative regimen, an anterior neurectomy appeared successful in approximately $70 \%$ of operated patients ${ }^{1-3}$. After a $>2.5$ years median follow up, a $61 \%$ long-term efficacy rate was recently demonstrated in this population ${ }^{3}$. In all, some three-quarters of ACNES patients are reasonably pain free if a stepwise regimen including injections followed by anterior neurectomy is followed. Such a treatment protocol may presently be considered standard treatment ${ }^{1-3,5,7}$.

Despite superior results compared to other treatment options for peripheral neuropathic pain treatment, approximately one-quarter of the eventually neurectomized patients do not respond to surgery or do experience recurrent abdominal wall pain over time ${ }^{1-4}$. These unsuccessful patients reported a substantial 7-8 pain score on a 0-10 pain intensity numeric rating scale ${ }^{3}$. No alternative treatment options apart from pharmacological pain strategies have been proposed for these populations ${ }^{8-10}$. In the SolviMáx center for abdominal wall and groin pain syndromes, alternative surgical treatment steps have been explored. If pain is still (or again) localized at the level of the abdominal wall, one may decide to inject anaesthetic agents in the painful area. If pain is temporarily relieved but recurs, a reexploration of the operated area may possibly allow for the identification and removal of persistent end twigs (secondary anterior neurectomy). Alternatively, removal of neurovascular bundles at the level of the posterior abdominal rectus fascia may theoretically offer relief (posterior neurectomy).

Aim of this retrospective study was to assess success rates of secondary surgical procedures in ACNES patients after a failed anterior neurectomy or who report recurrent pain after an initially successful anterior neurectomy. It is hypothesized that a substantia portion of these re-operated patients would benefit from secondary surgical procedures. 


\section{Materials and methods}

\section{General study information}

This retrospective observational study was conducted between July 2012 and January 2013 in Máxima Medical Center (MMC), a 543-bed teaching hospital situated in The Netherlands. Staff members of the surgical department of MMC ('SolviMáx' Center of Excellence for Abdominal Wall and Groin Pain) have since long expressed an interest in the diagnosis and treatment of chronic abdominal wall pain and groin pain syndromes including inguinal neuralgias and post caesarean section nerve entrapments. Increasing numbers of patients are evaluated and treated by a team of experts in this center ${ }^{1-5,11-19}$. It was judged that results of the present study were obtained as part of an evaluating and auditing process (patient response outcome measurement, PROM). Thus, approval by our local ethics committee was deemed not necessary.

\section{Patient selection}

All patients were evaluated on the basis of a standard history and physical examination. Selected patients were all previously diagnosed with ACNES using a set of common signs and symptoms ${ }^{1,20-23}$. ACNES is characterized by (1) a constant site of anterior abdominal tenderness that is superficially located with a small $(<2 \mathrm{~cm} 2)$ area of maximal intensity, (2) this point of maximal pain can be localized by the tip of 1 finger (trigger point), and (3) tenderness increases by abdominal muscle tensing using the Carnett's test. During this test, the trigger point is localized and palpated using a finger or a swab. Tenderness increases when the patient is asked to lift the head and abdominal muscles are tensed, suggesting its origin is probably located in the abdominal wall ${ }^{1,21,22}$. Typical findings such as a positive pinch test and the presence of local sensory disturbances in proximity to the trigger point strongly contributes to confirmation of the diagnosis ${ }^{1,4,19,20}$. Once ACNES is considered as a cause of chronic abdominal pain, the diagnosis is confirmed by local infiltration using a short-acting anesthetic agent. A simple treatment regimen including injections and a neurectomy in injection regimen refractive patients is subsequently offered $^{1}$.

Electronically stored data of all ACNES patients receiving an anterior neurectomy in the SolviMáx between January 2004 and February 2012 were entered in a database. An anterior neurectomy is defined as the first attempt to remove end twigs of intercostal nerves penetrating at the level of the anterior rectus muscle sheath via an open surgical procedure ${ }^{1-3}$. Pain levels using a 0-10 pain intensity numeric rating scale (PI-NRS) and a 6-point verbal categorical rating scale (VRS) were collected at various time points as previously reported (table 1$)^{1-4}$. A total of 214 anterior neurectomies were performed during the 8 year time period. Data of 33 patients were not obtained $(<18$ year $n=16$, loss to follow up $n=14$, deceased $n=3)^{3}$.Therefore, 181 primary anterior neurectomies were eligible for long term efficacy analysis.

From this database, two groups of ACNES patients were eligible for the present study. The first group consisted of patients who experienced persistent pain within the 3 months postoperative time period following the anterior neurectomy ('failures'). Persistent pain was defined as a less than $50 \%$ pain reduction (using PI-NRS) and a less than 2-point pain reduction (using VRS) compared to preoperative pain levels. The second group consisted of patients who were initially successfully treated (by definition a $\geq 50 \%$ reduction using PI-NRS, and/or $\geq 2$-point reduction using VRS) during the postoperative 3 months time period. However, pain recurred during follow up thereafter ('recurrent disease'). Recurrent pain was defined as a less than 50\% reduction (using PI-NRS) and a less than 2-point reduction (using VRS) compared to the level prior to the anterior neurectomy ${ }^{3}$.

Data of these two groups of patients (failures and recurrences) were studied in detail. Patients who decided to start again with conservative treatment regimens were excluded from analysis, mainly because these conservative treatment steps were received in facilities other than our center. As such, supervision on patient compliance and quality of care was deemed impossible. Moreover, the great variety of conservative treatment options including neurostimulation therapy, paravertebral blocks, and pharmacotherapy precludes a simple comparison with surgical therapy.

Subjects that did undergo additional surgery were interviewed by telephone by a researcher that was not involved in the surgical care (TvA). Perioperative pain levels (PINRS and VRS) and current status of pain after the secondary surgery were obtained as previously reported ${ }^{3}$. Type of surgical technique (reexploration or posterior neurectomy) and complications were extracted from the electronic patient file. 


\section{Table 1 Used pain measuring scales}

\section{Pain Intensity Numeric Rating Scale (PI-NRS)} No pain Excruciating pain

\begin{tabular}{l}
\hline 6-point Verbal categorical Rating Scale (VRS) for pain characteristics \\
$1-$ None \\
$2-$ Mild \\
3 - Discomforting \\
$4-$ Distressing \\
$5-$ Horrible \\
6 - Excruciating \\
\hline
\end{tabular}

\section{Secondary surgical treatment strategies}

Two surgical options were explored in persistently painful patients. If patient history and physical examination including local blocks suggested that the pain was due to the presence of previously unresected end twigs, the scar of the anterior neurectomy was reopened. Persistent neurovascular branches that were indeed found at the level of the anterior sheath of the abdominal rectus muscle were removed. This type of surgery is termed 'secondary anterior neurectomy'. In essence, this technique is similar to a primary anterior neurectomy $\mathrm{y}^{1-3}$.

An alternative procedure termed 'posterior neurectomy' was executed in the very same procedure if the secondary anterior neurectomy revealed no residual end twigs, or in a third procedure if a secondary anterior neurectomy again was not successful. During a posterior neurectomy, the intercostal nerve is identified at the level of the posterior sheath of the abdominal rectus muscle (figure 1). Characteristics of this surgical technique were not previously found in the surgical literature.

Similar to an anterior neurectomy, patients are operated in day care or are hospitalized for one night. The pain trigger point is preoperatively marked onto the skin. After induction of general anaesthesia, the scar is reopened and the anterior rectus aponeurosis (consisting of the external oblique sheath and the anterior part of the internal oblique sheath) is incised. The rectus muscle is retracted medially allowing inspection of the posterior rectus sheath. At this level, the posterior rectus sheath usually consists of the posterior part of the oblique muscle sheath and the transverse fascia. At the lateral border, the neurovascular bundle enters the space quite obliquely, running over the posterior fascia and entering the rectus muscle more medially. A $2-4 \mathrm{~cm}$ portion of the intercostal nerve is removed while the remaining proximal nerve ending is allowed to retract. Accompanying vessels are coagulated or ligated. Following the procedure, the wound is closed in layers as appropriate and the wound is infiltrated with a long acting local anaesthetic (i.e. $10 \mathrm{cc}$ of $0.25 \%$ bupivacaine). A posterior neurectomy usually lasts between 30-60 minutes.

This two-step surgical protocol in unsuccessfully operated or recurrent ACNES patients was not followed in two patients due to complicating factors as judged by the attending surgeon. As a consequence, these individuals were excluded from analysis.

Figure 1 Schematic (upper) and intraoperative (lower) impression of a secondary posterior neurectomy of the intercostal nerve penetrating at the level of the posterior rectus sheath in an ACNES patient (BMI 40,125 kg) after a previous unsuccessful anterior neurectomy at the level of Th10 (umbilicus) at the right side
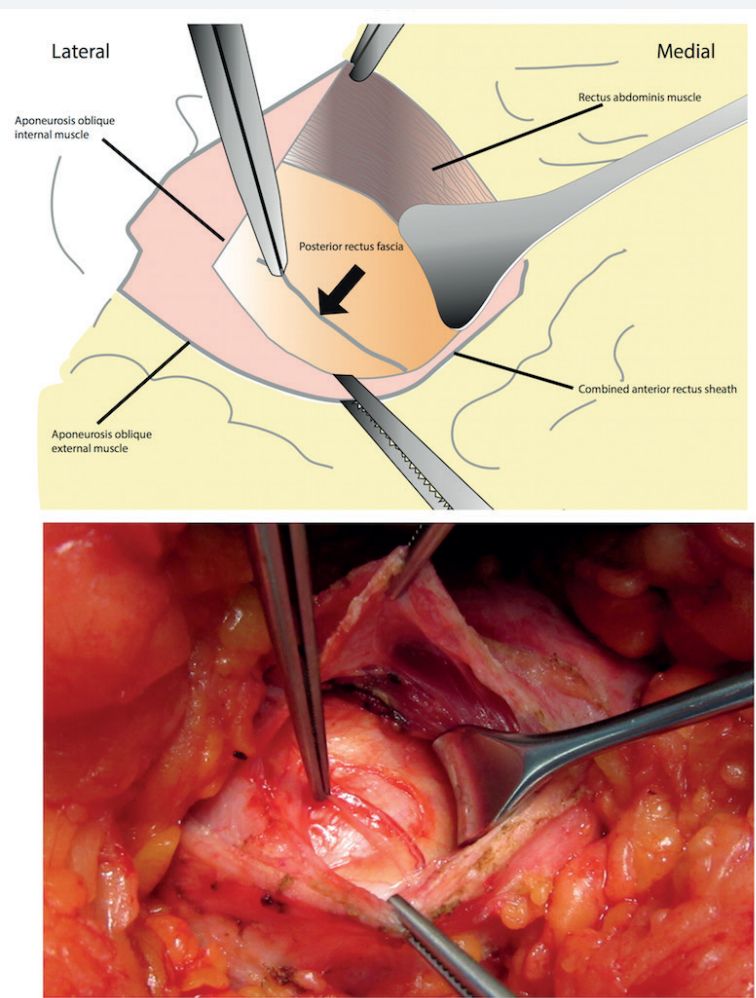
Data analysis

Data analysis was performed using SPSS 21.0 for Mac OS X. Changes in PI-NRS and VRS over time determined the success of operation. Fisher exact test was used to analyse the differences between surgical techniques or outcome. Differences in baseline characteristics between groups were tested using a $\chi 2$ test for categorical variables and a Student $t$ test (normal distribution) or Mann-Whitney test (skewed distribution) in case of continuous variables. A p-value $<0.05$ was considered statistical significant.

Figure 2 Flowchart of additional surgical treatment strategy in patients with either failure or recurrent disease after a primary anterior neurectomy

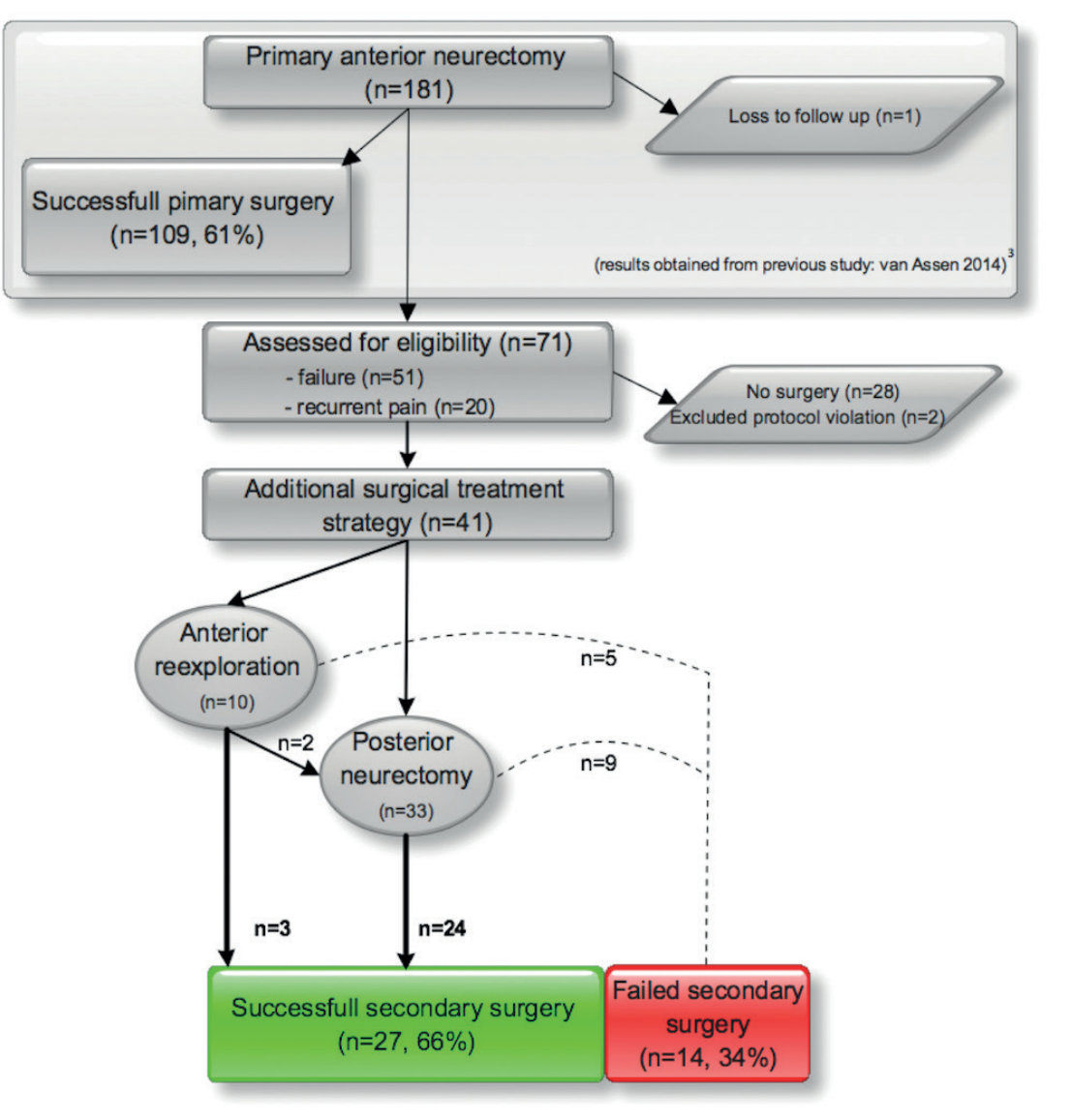

\section{Results}

Patient selection

As reported in the materials and methods, 181 primary anterior neurectomies performed in SolviMáx between January 2004 and February 2012 were analysed for long term efficacy ${ }^{3}$. A total of 71 patients thereof met inclusion criteria of the present study (figure 2). There was no loss to follow up.

This group of 71 individuals contained 51 immediate failures after an anterior neurectomy and 20 patients with recurrent disease after an initially successful anterior neurectomy. 28 patients ( 24 failures and 4 recurrences) opted for a conservative treatment consisting of a wait-and-see policy supported by medication. The two-step secondary surgical protocol was not followed in 2 ( 1 failure, 1 recurrent pain). Consequently, the present study reports on data obtained from 41 patients (26 failures and 15 recurrences).

\section{Surgical results}

The majority of these 41 subjects was female $(n=29,71 \%$; male $n=12)$. Median age was $43 \pm 16$ years (range 20-77). Patient characteristics were not different compared to the conservatively treated group $(n=28$, female $89 \%, p=0.08$; age $52 \pm 17$ years; range $20-77$, $\mathrm{p}=0.10)$.

A re-exploration (secondary anterior neurectomy) was executed in 10 patients leading to success in 3. A posterior neurectomy (as a third operation) after a failed secondary exploration in 2 additional patients proved successful in both. The re-exploration was not successful in the other half $(\mathrm{n}=5)$ of these patients. No additional explorations were performed in these 5 individuals. Of these, 4 decided to undergo treatment regimens provided by alternative health care facilities (pain clinic $n=2$, orthomanual therapy $n=1$, third opinion $\mathrm{n}=1$ ). The fifth patient accepted the current pain level (PI-NRS $=5)$ as it was better compared to preoperatively (PI-NRS $=8$ ).

A posterior neurectomy was executed in the remaining 31 patients leading to success in $22(22 / 31,71 \%$ success rate). Therefore, the secondary surgical treatment protocol was overall successful in two thirds of the operated patient group (66\%, 27/41; median 25 months (range, 3-49) follow-up). PI-NRS scores of successful and unsuccessful subjects before secondary surgery protocol were comparable (successful 8.07, SD 1.33, range 5-10 vs. unsuccessful 8.00 , SD 1.18, range 5-10; $\mathrm{p}=0.82$ ). 
When focusing on the group of immediate failures after the anterior neurectomy, 26 underwent secondary surgery. Surgical treatment was successful in 50\% (13/26). In contrast, additional surgery appeared successful in 14 of 15 (93\%) patients with a recurrence. Therefore, secondary surgery was more effective for recurrent pain than for residual pain ( $14 / 15$ vs. $13 / 26, \mathrm{p}=0.01)$.

Complications included one wound abscess requiring a surgical drainage procedure and one wound infection that was effectively treated with a seven-day course of antibiotics.

\section{Discussion}

The present study in ACNES patients demonstrating severe pain after a failed anterior neurectomy or who experienced recurrent pain after an initially successful neurectomy, indicates that additional surgical procedures may be beneficial in two-thirds of the population. Furthermore, secondary surgery for recurrent ACNES pain is more effective compared to surgery for patients with residual pain. This difference may possibly be explained by disinhibition of central neural pathways in ACNES patients with residual pain $^{24}$. Conversely, one third does not respond favourably. Medication may be of benefit in these individuals ${ }^{8,9}$. Alternatively, neurostimulation therapy or paravertebral blocks may be explored as suggested by a number of case report studies ${ }^{10,25-28}$. Pain physicians who are specialized in treating these chronic peripheral neuropathic pain populations should also explore these treatment options prior to deciding on secondary surgery.

Data of the present study allow for a rough calculation of the overall ACNES treatment success rate in a dedicated institution. Recently, treatment efficacy of a combined stepwise approach including injections followed by surgery (if required) was reported ${ }^{1-4}$. Interestingly, a regimen of just consecutive local trigger point injections was long-term effective in $33 \%$ of investigated patients ${ }^{1}$. Surgical neurectomy was median term beneficial in $70 \%$ of injection-recalcitrant patients ${ }^{1,2}$. Despite occasional recurrences, $61 \%$ of a large ACNES population remained satisfied on the long-term ${ }^{3}$. Although data were generated in different study populations, these results seem to confirm the great potential of a standard treatment protocol. The treatment protocol that is currently proposed consisting of injection therapy (step I) followed by an anterior neurectomy (step II) supplemented with secondary surgical options (step III) may lead to a $90 \%$ success rate (figure 3 ).
Figure 3 Flowchart of the total treatment protocol developed in recent years based on an imaginary 100 patient cohort.

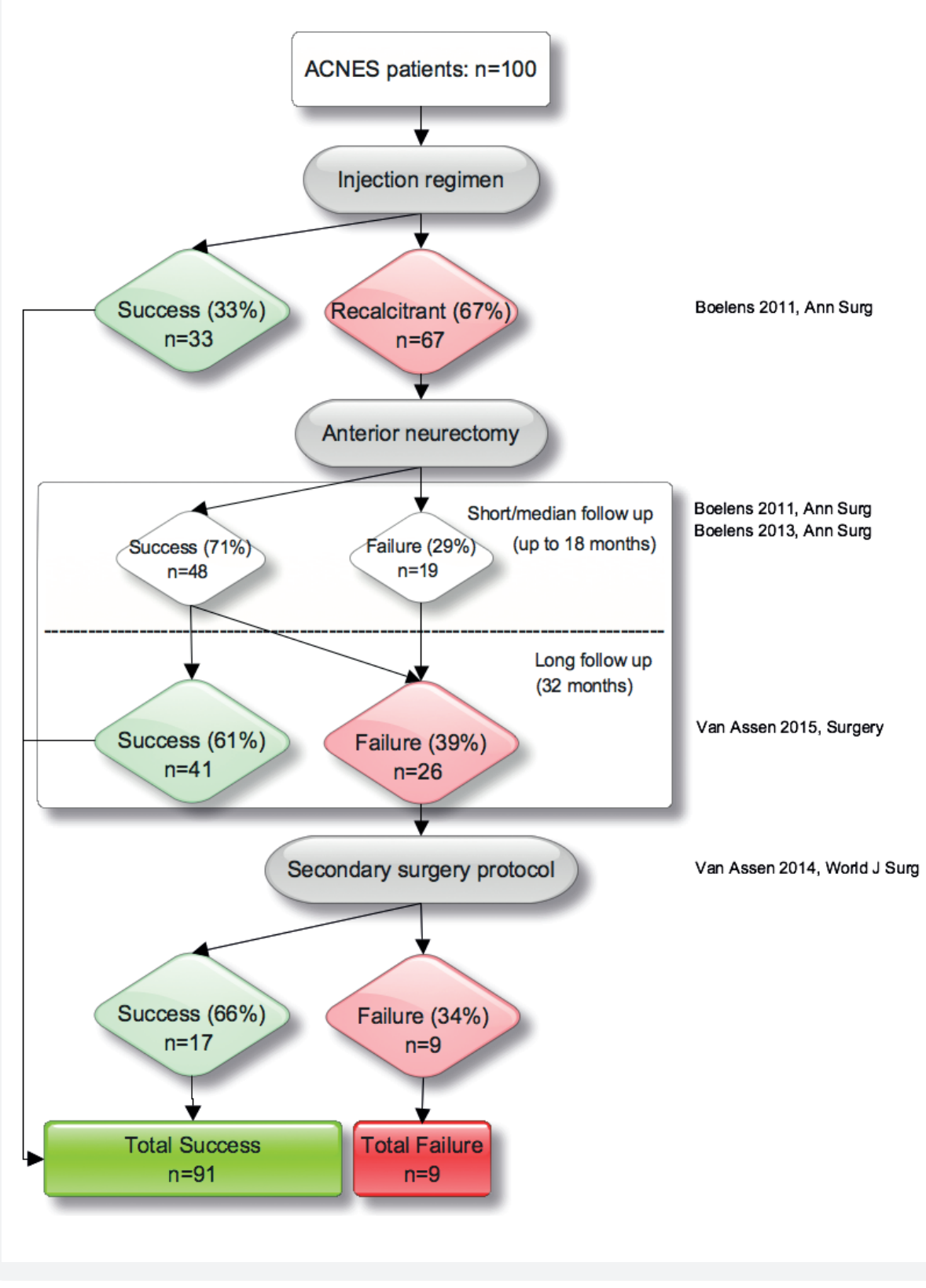


Initially, scepticism was present regarding the potential salutary effect of secondary surgery including additional neurectomies in persistently painful patients. Despite our reservation however, secondary surgical attempts appeared successful in the majority of patients. An important issue during the surgical procedure such as the surgeon's role requires addressing. For instance, nerve ends may have been missed during the anterior neurectomy. It should be appreciated that removing end twigs is a matter of balance. It makes sense that only nerves causing the pain are resected favouring a limited exploration. Based on the premise that a one-trigger-point-ACNES is a pain syndrome caused by entrapment of twigs of just one intercostal nerve branch, the surgeon should identify all branches of this specific nerve. However, these structures may look perfectly normal. Therefore, visual inspection does not help the surgeon to distinguish normal from entrapped branches. On the other hand, a policy of 'not missing the causal nerve at any rate' may lead to a more extensive nerve resection including (portions of) neighbouring intercostal branches. This approach potentially enhances the chance of post dissection pain and neuroma formation. Residual pain is thus possibly explained by a too limited resection during the anterior neurectomy.

Secondly, which surgical technique can be utilized once the plane superficial to the anterior rectus sheath is judged devoid of penetrating nerve endings and a re-exploration is on the verge of failing? One option is to explore the alleged entrapped intercostal nerves at a more proximal level. Interruption of the intercostal nerve at the level of the posterior rectus sheath entrance was found effective in a substantial portion of persistently painful patients. In fact, in the present paper we have described a novel surgical procedure that is not reported previously. However, the value of a posterior neurectomy as a surgical alternative equal to an anterior neurectomy in patients refractory to injections is questionable and is therefore currently under investigation.

The present study harbours flaws including retrospectively accumulated pain scores. Interestingly, a recent study evaluating differences in prospectively versus retrospectively generated pain data found only a negligible and clinically irrelevant influence on recall bias in this setting ${ }^{3}$. Another flaw is the lack of specifics and follow up data of patients that opted for conservative treatment. The number of patients may also seem limited. However, this study is probably the only one as other studies on secondary surgery for ACNES were not identified in the literature.

\section{Conclusion}

In the search for a sensible and logical stepwise approach in the treatment of ACNES, additional surgical strategies are beneficial in two-thirds of patients reporting pain after an anterior neurectomy. Patients with recurrence of pain do better than patients with residual abdominal wall pain. 


\section{References}

1. Boelens OB, Scheltinga MR, Houterman S, et al. Management of anterior cutaneous nerve entrapment syndrome in a cohort of 139 patients. Ann Surg. 2011; 254: 1054-1058.

2. Boelens OB, van Assen T, Houterman S, et al. A double-blind, randomized, controlled trial on surgery for chronic abdominal pain due to anterior cutaneous nerve entrapment syndrome. Ann Surg. 2013; 257: 845-849.

3. van Assen T, Boelens OB, van Eerten PV, et al. Long-term success following an anterior neurectomy in patients with an abdominal cutaneous nerve entrapment syndrome. Surgery. 2015; 157: 137-43.

4. Boelens OB, Scheltinga MR, Houterman S, et al. Randomized clinical trial of trigger point infiltration with lidocaine to diagnose anterior cutaneous nerve entrapment syndrome. Br J Surg. 2013; 100: 217-221.

5. Scheltinga MR, Boelens OB, Tjon ATWE, et al. Surgery for refractory anterior cutaneous nerve entrapment syndrome (ACNES) in children. J Pediatr Surg. 2011; 46: 699-703.

6. Towfigh S, Anderson S, Walker A. When it is not a spigelian hernia: abdominal cutaneous nerve entrapment syndrome. Am Surg. 2013; 79: 1111-1114.

7. Srinivasan R, Greenbaum DS. Chronic abdominal wall pain: a frequently overlooked problem. Practical approach to diagnosis and management. Am J Gastroenterol. 2002; 97: 824-830.

8. Finnerup NB, Otto M, McQuay HJ, et al. Algorithm for neuropathic pain treatment: an evidence based proposal. Pain. 2005; 118: 289-305.

9. Attal N, Cruccu G, Baron R, et al. EFNS guidelines on the pharmacological treatment of neuropathic pain: 2010 revision. Eur J Neurol. 2010; 17: 1113-e1188.

10. Cruccu G, Aziz TZ, Garcia-Larrea L, et al. EFNS guidelines on neurostimulation therapy for neuropathic pain. Eur J Neurol. 2007; 14: 952-970.

11. van Assen T, Boelens OB, Kamphuis JT, et al. Construction and validation of a questionnaire distinguishing a chronic abdominal wall pain syndrome from irritable bowel syndrome. Frontline gastroenterol. 2012; 3: 288-294.

12. Loos MJ, Roumen RM, Scheltinga MR. Chronic sequelae of common elective groin hernia repair. Hernia. 2007; 11: 169-173.

13. Loos MJ, Roumen RM, Scheltinga MR. Classifying post-herniorrhaphy pain syndromes following elective inguinal hernia repair. World J Surg. 2007; 31: 1760-1765; discussion 1766-1767.
14. Loos MJ, Scheltinga MR, Mulders LG, et al. The Pfannenstiel incision as a source of chronic pain. Obstet Gynecol. 2008; 111: 839-846.

15. Loos MJ, Scheltinga MR, Roumen RM. Surgical management of inguinal neuralgia after a low transverse Pfannenstiel incision. Ann Surg. 2008; 248: 880-885.

16. Loos MJ, Scheltinga MR, Roumen RM. Tailored neurectomy for treatment of postherniorrhaphy inguinal neuralgia. Surgery. 2010; 147: 275-281

17. Loos MJ, Verhagen T, Scheltinga MR, et al. A randomised controlled trial of injection therapy versus neurectomy for post-herniorrhaphy inguinal neuralgia: rationale and study design. Hernia. 2010; 14: 593-597.

18. Roumen RM, Scheltinga MR. [Abdominal intercostal neuralgia: a forgotten cause of abdominal pain]. Ned Tijdschr Geneeskd. 2006; 150: 1909-1915.

19. van Assen T, de Jager-Kievit JW, Scheltinga MR, et al. Chronic abdominal wall pain misdiagnosed as functional abdominal pain. J Am Board Fam Med. 2013; 26: 738-744.

20. Applegate WV. Abdominal Cutaneous Nerve Entrapment Syndrome (ACNES):

21. A Commonly Overlooked Cause of Abdominal Pain. Perm J. 2002; 6: 20-27.

22. Carnett J. Intercostal neuralgia as a cause of abdominal pain and tenderness. Surg Gynecol Obstet. 1926; 42: 8.

23. Greenbaum DS, Greenbaum RB, Joseph JG, et al. Chronic abdominal wall pain. Diagnostic validity and costs. Dig Dis Sci. 1994; 39: 1935-1941.

24. Thomson WH, Dawes RF, Carter SS. Abdominal wall tenderness: a useful sign in chronic abdominal pain. Br J Surg. 1991; 78: 223-225.

25. Salter MW. Deepening understanding of the neural substrates of chronic pain. Brain 2014; 137: 651-653.

26. Oosterhof J, Wilder-Smith OH, Oostendorp RA, et al. Different mechanisms for the shortterm effects of real versus sham transcutaneous electrical nerve stimulation (TENS) in patients with chronic pain: a pilot study. J Pain Palliat Care Pharmacother. 2012; 26: 5-12.

27. Cohen SP, Sireci A, Wu CL, et al. Pulsed radiofrequency of the dorsal root ganglia is superior to pharmacotherapy or pulsed radiofrequency of the intercostal nerves in the treatment of chronic postsurgical thoracic pain. Pain physician. 2006; 9: 227-235.

28. Philip CN, Candido KD, Joseph NJ, et al. Successful treatment of meralgia paresthetica with pulsed radiofrequency of the lateral femoral cutaneous nerve. Pain physician. 2009; 12: 881-885.

29. Werner MU, Bischoff JM, Rathmell JP, et al. Pulsed radiofrequency in the treatment of persistent pain after inguinal herniotomy: a systematic review. Reg Anesth Pain Med. 2012; 37: $340-343$ 


\section{Chapter 9}

Summarizing discussion, conclusions and

future perspectives 


\section{Summarizing discussion}

Abdominal wall pain syndromes such as the anterior cutaneous nerve entrapment syndrome (ACNES) have never received the attention that is deserved. Physicians from different specialties and countries have reported on this syndrome in the preceding century $^{1-8}$. Unfortunately, these few studies have just resulted in a basic level of knowledge on abdominal wall pain syndromes. For instance, a 2006 survey in Dutch surgeons $(n=425)$ and surgical interns $(n=82,35 \%$ response rate) revealed that $85 \%$ of respondents did not even know the syndrome at all. Of senior surgeons with $>10$ years of experience, just $18 \%$ was ever confronted with abdominal wall pain such as ACNES. Moreover, less than $9 \%$ of surgical interns was up-to-date with symptoms of the syndrome ${ }^{7}$. These numbers are unacceptably low, considering that exposure to this patient category in a surgical department may be high. Knowledge among non-surgical specialties may even be more limited.

ACNES is a typical neuropathic entrapment syndrome that is generally associated with high pain levels. As a consequence, each patient will consult a doctor. If not recognized (not unlikely considering the marginal awareness), a series of unnecessary costly diagnostic evaluations may be ordered by general practitioners and hospital specialists ${ }^{6,9}$. In one study, 418 diagnostic procedures were executed in 100 CAWP patients ${ }^{10}$. A 1994 report demonstrated that an average workup amounted to a 680 US\$ per CAWP patient ${ }^{5}$. Moreover, an astonishing 6727 US\$ per patient was invested in futile diagnostic testing and hospital charges before arriving at the correct diagnosis in a 2001 CAWP study ${ }^{11}$. These data indicate that the economic burden of these entities is substantial.

In current practice with nearly unlimited diagnostic options, the need for a proper physical examination in chronic pain patients may seem of minor importance. However, abdominal wall pain syndromes including ACNES are characteristic 'clinical entities' that may be diagnosed with the doctor's ears, eyes and fingers. In patients suffering from ACNES, a continuous sharp or burning pain with exacerbations dominates the picture ${ }^{12}$, ${ }^{13}$. Maximal tenderness is usually located on a 'patient-characteristic' spot within lateral boundaries of the rectus abdominis muscle. Onset of pain is often acute and is occasionally caused by previous surgery, pregnancy, sports or an abrupt movement. However, a clear cause is not found in about half of the patients ${ }^{12}$. Provoking factors are certain body poses and activity. Symptoms that mimic gastrointestinal organ dysfunction ('pseudo-visceral') can also be experienced in some individuals.

As ACNES is generally considered a typical 'clinical syndrome', physical examination is a most crucial step in the diagnosis. A constant site of abdominal tenderness with a small ( $<2 \mathrm{~cm}^{2}$, 'finger tip') area of maximal intensity situated is located at the level of the rectus abdominis muscle. Tenderness increases when these abdominal muscles are tensed while the patient lifts the head (Carnett's test). Local sensory disturbances of the skin are frequently present. A so-called 'pinch' test (squeezing an overlying skin fold containing the patient's skin and subcutaneous fat between thumb and index finger) will often unvei a very sensitive skin covering the area of interest compared to the contralateral side. If the diagnosis ACNES is deemed likely, the physician may perform a local pain point infiltration using lidocain or bupivacaine as a means to confirm the diagnosis. A regimen of consecutive abdominal wall injections is effective in some one-thirds of patients with ACNES $^{12,13}$. Surgical neurectomy is worthwhile in approximately two-thirds of the injection regimen-refractory patients. In total, eighty percent of an ACNES population reported total or substantial pain relief on after a median 18 months follow-up following this approach ${ }^{12}$. Chapter 1 provides a more detailed description of the background, diagnosis and treatment of ACNES.

The first main subject of this thesis focused on epidemiology and economic impact of the syndrome. Patients who are labeled with a 'diagnosis of exclusion' such as the irritable bowel syndrome (IBS) may possibly suffer from a neglected abdominal wall syndrome such as ACNES. An IBS population is heterogeneous and harbors a variety of abdominal symptoms. According to the Rome III criteria, the diagnosis IBS is based on clinical symptoms in the absence of structural or tissue abnormalities. It is surprising that no criteria about physical examination are included. IBS patients by definition repor abdominal complaints at least 3 days per month in the preceding 3 months. Moreover, the diagnosis is probable if two or more of the following criteria are fulfilled. The onset is associated with a change in frequency and/or appearance of stool, whereas symptoms are attenuated after defecation..$^{14}$ It was hypothesized in the present thesis that some ACNES patients may be misdiagnosed as IBS and ACNES share symptoms. Chapter 2 reports on the construction and validation of a questionnaire that is able to distinguish patients with ACNES from IBS. A simple 18-item ( 0 - 18 points) questionnaire showed a 0.94 sensitivity combined with a 0.92 specificity at a 10 points cut-off level. 
The study reported in chapter 3 demonstrates that ACNES may be diagnosed in some patients currently being treated for functional abdominal complaints such as IBS. In a cross-sectional analysis, 4 Dutch primary care practices employing physicians who were unaware of the existence of ACNES were selected. A total of 535 patients $>18$ years old who were registered with a functional abdominal pain diagnosis were approached when they were symptomatic to complete an 18 -item questionnaire. From the 167 symptomatic responders, 18 individuals scoring above the earlier identified 10-point cutoff point underwent a diagnostic evaluation to establish their final diagnosis. Half of them were found to have a CAWP syndrome. Six subjects harbored characteristics that are typically associated with ACNES, corresponding to a $3.6 \%$ prevalence (95\% CI, 1.7-7.6) in this responding population with alleged IBS $(n=167)$. Based on a Dutch prevalence of IBS and estimations on percentage of IBS patients consulting a doctor, a minimum half a million Dutch individuals were found to seek medical help ${ }^{15-18}$. On the basis of these assumptions, some 20 thousand Dutch individuals diagnosed as having functional abdominal pain in fact suffer from ACNES (95\% CI, 9.500 - 42.000). Numbers of ACNES patients in primary care practices may even be higher as the present survey only focused on a small portion of the patient population. Findings in this study may be an eye-opener for general practitioners.

The aim of the observational study that is reported in chapter 4 was to calculate the incidence of ACNES in patients presenting with abdominal pain in the emergency department (ED) of a Dutch teaching hospital. Together with obtained incidence numbers of patients evaluated in the surgical outpatient department a general incidence rate could be determined. Data sets of all patients visiting Máxima Medical Center's (MMC) ED in 2011-2012 were searched for key terms 'ACNES', 'intercostal neuralgia' or 'abdominal wall pain'. Files of potential patients living within hospital's adherence area were checked using accepted criteria indicating the presence of ACNES. Disease incidence was calculated as a ratio to the hospital's adherence data. The ACNES MMC 2013's incidence in patients living within the adherence area and evaluated in the surgical outpatient department was also calculated. During the 2-year study period, a total of 88 patients belonging to MMC's adherence area were diagnosed with ACNES leading to a mean 22/100.000 ACNES yearly incidence rate on the ED. This finding indicates that approximately 1 to 50 patients presenting with acute abdominal pain on an ED is found to suffer from ACNES. Furthermore, a 35/100.000 outpatient department ACNES incidence rate was calculated in the year of 2013. Combining these two ratios, a 1:1.800 total ACNES incidence in the general population was obtained.

Some studies have attracted attention to an exceedingly high health care consumption in ACNES populations. However, these investigations were performed several decades ago in countries that differ from the Dutch health care system. An analysis of direct medical and indirect productivity costs in the year preceding the diagnosis in an ACNES patient population in a single hospital (MMC) was reported in chapter 5. Patients who were referred from outside the hospital's adherence area and who received the new diagnosis ACNES between September 2012 and August 2014 in MMC were eligible for inclusion. Study subjects were invited to complete a medical consumption questionnaire ( $i \mathrm{MCQ}$ ) and a productivity consumption questionnaire ( $i \mathrm{PCQ})$. Numbers of health care consumption obtained from the $\mathrm{MCQ}$ were multiplied with unit cost prices to attain direct diagnostic and therapeutic costs. Productivity losses were calculated by adding up the costs of absenteeism, presenteeism and unpaid work loss. Ninety-four patients fulfilled study criteria (female $n=74,79 \%$, mean age 41 SD 17). Total direct health care costs in the year preceding the diagnosis was estimated to approximate $€ 5,400$ per patient. Extrapolation to total number of years of doctor's delay resulted in direct medical costs of $€ 16,600$ per patient. A total of $€ 13,800$ of indirect costs per patient in the year before diagnosis was calculated, leading to a total of $€ 19,200$ in the year preceding the correct diagnosis. It is concluded that patients with ACNES are avid health care consumers in the year preceding their diagnosis. Earlier recognition will likely reduce unnecessary direct and indirect costs associated with the diagnostic and treatment approach.

The second main subject of this thesis is studying surgical treatment options in ACNES. Although approximately one third of ACNES patients is long-term successfully treated with an injection regimen, most patients experience recurrent complaints after such a conservative regimen. A surgical neurectomy was effective in about two thirds of the injection regimen refractory patients. ${ }^{19}$ Favorable results of a surgical neurectomy were, however, not yet demonstrated in a randomized controlled fashion. In chapter $\mathbf{6}$, adult patients diagnosed with ACNES were randomized to receive an anterior neurectomy or a sham (placebo) procedure via an open surgical procedure in day care. Both patients and principal investigator were blinded to the nature of surgery. Pain was recorded prior to surgery and 6 weeks postoperatively. A $\geq 50 \%$ reduction on a visual analogue (VAS) 
scale and/or 2 points drop on a verbal rating scale (VRS) were considered 'successful responses. Forty-four patients participated between August 2008 and December 2010. In the neurectomy group, 16 of 22 patients reported a successful response. In contrast, significant pain reduction was obtained in just 4 patients in the sham group $(\mathrm{p}=0.001)$. This study demonstrates that pain in most ACNES patients is caused by terminal branches of intercostal nerves.

An anterior neurectomy may seem successful but follow-up was short and populations were small. The primary aim of a cross sectional report (chapter 7) was to determine the long-term success rate of surgery in a large ACNES population. Patients who underwent a primary anterior neurectomy between January 2004 and February 2012 were investigated. Pain scores were obtained before and after surgery, and at the moment of follow up using a pain-intensity numeric rating scale (PI-NRS 0-10) and a 6-point VRS scale. Success was defined as a $\geq 50 \%$ PI-NRS reduction or $\geq 2$ point VRS reduction. Data of 181 neurectomies in 154 individuals were available for analysis. Short-term (1-3 months postoperative) success was 70\%. After a mean 32 months (range 3-93) followup, a success rate of $61 \%$ was found. Consequently, a $16 \%(n=20)$ recurrence rate was observed. It was concluded that surgery is long-term successful in roughly two of three operated ACNES patients.

Although the currently proposed treatment protocol (injections supplemented with an anterior neurectomy) is long-term successful in most ACNES patients, alternative treatment options for the remaining unresponsive quarter were never investigated. In chapter 8 , the efficacy of secondary surgery including a re-exploration or a posterior neurectomy in patients reporting recurrent pain after initially successful surgery or following an immediately failed anterior neurectomy is studied. Adult patients with residual pain after an anterior neurectomy (failures) or with recurrent pain after initially successful surgery (recurrences) were selected from a database of 181 surgical neurectomies that were performed in MMC between 2004 and 2012. Following a reexploration or a posterior neurectomy, pain was scored using a pain intensity numeric rating scale (PI-NRS 0-10) and a six-point verbal category rating scale (VRS). Success was defined as a $\geq 50 \%$ PI-NRS reduction and/or $\geq 2$ point VRS reduction. At follow-up, 51 patients reported unacceptable pain levels following an anterior neurectomy, whereas 20 developed recurrent abdominal wall pain. Of these 71 unsuccessful patients, 41 underwent secondary surgery including a re-exploration $(n=10)$ or a posterior neurectomy $(n=31)$. After a median 25-month follow-up, these secondary surgical treatment regimens proved successful in 66\%. Patients with recurrent pain did better (14/15) than patients who were immediate failures after the anterior neurectomy $(13 / 26, \mathrm{p}=0.01)$. It was concluded that secondary surgery is worthwhile in ACNES patients who did not respond to a treatment protocol including injections supplemented with an anterior neurectomy. 


\section{Conclusions of this thesis}

1. An 18-item questionnaire is a useful tool to prospectively identify patients with abdominal wall pain who were earlier diagnosed as having functional abdominal pain (sensitivity 0.92 , specificity 0.94 ).

2. A clinically relevant portion of patients diagnosed with a functional abdominal pain diagnosis in a general practitioner's setting actually suffers from ACNES (prevalence 3.6\%, CI $1.7-7.6 \%$ ).

3. The incidence of acute ACNES patients in an emergency department (ED) of a regional teaching hospital is $22 / 100.000$ per year indicating that approximately $2 \%$ of patients presenting on an $\mathrm{ED}$ with acute abdominal pain suffers from ACNES. The general incidence of ACNES is estimated at 1:1.800 suggesting that, in the Netherlands alone, some 9.500 unique patients may be diagnosed with ACNES yearly.

4. Patients with ACNES are avid health care consumers in the year preceding their diagnosis ( $€ 5,400$ per patient). Extrapolation on the basis of years of doctor's delay resulted in direct medical costs of $€ 16,600$ per patient. As a total of $€ 13,800$ of indirect costs per patient in the year before diagnosis was calculated, a total of $€ 19,200$ in the year preceding the correct diagnosis was spent per ACNES patient.

5. Neurectomy of intercostal nerve endings at the level of the abdominal wall effectively reduces pain in ACNES patients failing a conservative regimen. A 70\% short-term success rate and a $61 \%$ long-term success rate after a primary anterior neurectomy are attained.

6. Secondary surgery including re-explorations and posterior neurectomies are successful in two-thirds of ACNES patients failing a combined injection-anterior neurectomy regimen.

\section{Future perspectives}

Basic information regarding incidence, prevalence, diagnosis and treatment for ACNES is sketchy. Efforts initiated in our SolviMáx expertise center are aimed at closing basic gaps in this knowledge. A growing number of referred patients provides us with a unique possibility to answer important future research questions. A 'no nonsense' practical approach in SolviMáx gave way to the development of a standard treatment protocol in ACNES that appeared to suit most patients suffering from this abdominal wall pain syndrome. However, a small minority of patients did not benefit, no matter what was done.

One future research interest is related to studying this group of non-responders. Important questions such as 'how did they respond on injection therapy?', 'what was their duration of complaints?', 'which signs during physical examination were present?' must be answered. By possibly recognizing patterns in responders versus non-responders, prognostic factors may be identified. Potential subtypes of ACNES may exist. Children with ACNES potentially form a subgroup that may behave differently ${ }^{8}$. Etiology of adolescent ACNES is possibly more mechanical leading to just symptoms at the level of the anterior branch of the intercostal nerve, compared to an adult population. In an ideal situation, prognostic factors are determined on an individual level leading to a patientspecific approach. Furthermore, as many patients report (pseudo) visceral complaints that recover after a successful ACNES treatment, the role of segmental relations (visceroafferent and vice versa) should be investigated and elucidated.

The current thesis also focused on the burden of ACNES in terms of societal cost. However, studies also demonstrated a substantial inability to fulfill normal daily activities as reflected by high rates of sick leave and efficiency losses of paid work. Moreover, it is likely that a delay in diagnosis and inappropriate treatment will also seriously affect quality of life. The role of psychological factors in ACNES should be studied more intensively. Future studies are required to attain a complete evaluation of altered health status in ACNES patients.

A specific quality of a non-academic center such as MMC is a somewhat down-toearth and pragmatic approach of most medical issues. As such, a frequently overlooked 
abdominal pain syndrome lacking a structured diagnostic approach and treatment protocol was identified. Subsequently, a scientific program focused on improving recognition, diagnosis and treatment was developed. Several contributions in leading journals enabled physicians to follow a proposed step-by-step treatment protocol. Surgical treatment results were superior compared to treatment strategies in other chronic peripheral neuropathic pain populations ${ }^{20,21}$. All these efforts were established in a relatively short time frame. However, it must be appreciated that a number of basic fundamental questions require answering. Future basic research, together with an academic partner, should focus on nerve anatomy and pathophysiology of the syndrome. According to a logical stepwise research approach, reverting to basal research may seem as 'one-step-back'. However, results from these basic studies may allow for new ideas expanding knowledge on classification and treatment of this intriguing syndrome. An improved understanding of nerve anatomy may give clues for the development of alternative surgical techniques. As showed in this thesis, some patients benefitted from a novel surgical technique termed a 'posterior neurectomy'. The intercostal nerve is identified at the level of the posterior sheath of the abdominal rectus muscle. A successful response in a portion of these patients suggests that the posterior rectus sheath may also serve as a possible site for entrapment. Consequently, it is hypothesized that a posterior neurectomy may be a better choice as initial surgical step in injection regimen refractory patients. With expanding knowledge regarding anatomy of the intercostal nerve, more proximal neurectomies using a laparoscopic approach may be worthwhile in some patients. These alternative options should be studied in the near future.

It is appreciated that the present future perspectives are exclusively presented from a surgical point of view. Additional treatment options lie outside of the surgical spectrum. Medication may be of benefit in some ACNES patients but structured studies are currently lacking ${ }^{20,21}$. Neurostimulation therapy or paravertebral blocks may also be explored as suggested by a number of case report studies, as well as manual therapy $\mathrm{y}^{22-26}$. In our practice, these treatment steps are currently investigated once an unsuccessful surgical treatment is at hand. But it may be worthwhile to initiate these strategies as a first step in the treatment process.

\section{References}

1. Carnett J. Intercostal neuralgia as a cause of abdominal pain and tenderness. Surg Gynecol Obstet. 1926; 42: 8.

2. Ranger I, Mehta M, Pennington M. Abdominal wall pain due to nerve entrapment. Practitioner. 1971; 206: 791-792.

3. Thomson H, Francis DM. Abdominal-wall tenderness: A useful sign in the acute abdomen. Lancet. 1977; 2: 1053-1054.

4. Gallegos NC, Hobsley M. Recognition and treatment of abdominal wall pain. JR Soc Med. 1989; 82: 343-344.

5. Greenbaum DS, Greenbaum RB, Joseph JG, et al. Chronic abdominal wall pain. Diagnostic validity and costs. Dig Dis Sci. 1994; 39: 1935-1941.

6. Applegate WV. Abdominal Cutaneous Nerve Entrapment Syndrome (ACNES): A Commonly Overlooked Cause of Abdominal Pain. Perm J. 2002; 6: 20-27.

7. Roumen RM, Scheltinga MR. [Abdominal intercostal neuralgia: a forgotten cause of abdominal pain]. Ned Tijdschr Geneeskd. 2006; 150: 1909-1915.

8. Scheltinga MR, Boelens OB, Tjon ATWE, et al. Surgery for refractory anterior cutaneous nerve entrapment syndrome (ACNES) in children. J Pediatr Surg. 2011; 46: 699-703.

9. Srinivasan R, Greenbaum DS. Chronic abdominal wall pain: a frequently overlooked problem. Practical approach to diagnosis and management. Am J Gastroenterol. 2002; 97: $824-830$

10. Hershfield NB. The abdominal wall. A frequently overlooked source of abdominal pain. J Clin Gastroenterol. 1992; 14: 199-202.

11. Thompson C, Goodman R, Rowe WA. Abdominal wall syndrome, a costly diagnosis of exclusion. Gastroenterol. 2001; 120: A637.

12. Boelens OB, Scheltinga MR, Houterman S, et al. Management of anterior cutaneous nerve entrapment syndrome in a cohort of 139 patients. Ann Surg. 2011; 254: 1054-1058.

13. Boelens OB, Scheltinga MR, Houterman S, et al. Randomized clinical trial of trigger point infiltration with lidocaine to diagnose anterior cutaneous nerve entrapment syndrome. Br J Surg. 2013; 100: 217-221.

14. Drossman M. Rome criteria. [Web page] 2011; Available from: http://www.romecriteria. org/.

15. Drossman DA, Whitehead WE, Camilleri M. Irritable bowel syndrome: a technical review for practice guideline development. Gastroenterol. 1997; 112: 2120-2137. 
16. Statistiek CBvd. Bevolkingsteller. [web page] [cited 2012; Available from: http://www.cbs. nl/nl-nl/menu/themas/bevolking/cijfers/extra/bevolkingsteller.htm.

17. Talley NJ, Weaver AL, Zinsmeister AR, et al. Onset and disappearance of gastrointestinal symptoms and functional gastrointestinal disorders. Am J Epidemiol. 1992; 136: 165-177.

18. Van der Velden J. Een nationale studie naar ziekten en verrichtingen in de huisartspraktijk. Basisrapport; Morbiditeit in de huisartspraktijk. 1991.

19. Applegate W. Abdominal Cutaneous Nerve Entrapment syndrome (ACNES): A Commonly Overlooked Cause Of Abdominal Pain. Perm J. 2002; 6: 20-27.

20. Attal N, Cruccu G, Baron R, et al. EFNS guidelines on the pharmacological treatment of neuropathic pain: 2010 revision. Eur J Neurol. 2010; 17: 1113-e1188.

21. Finnerup NB, Otto M, McQuay HJ, et al. Algorithm for neuropathic pain treatment: an evidence based proposal. Pain. 2005; 118: 289-305.

22. Cohen SP, Sireci A, Wu CL, et al. Pulsed radiofrequency of the dorsal root ganglia is superior to pharmacotherapy or pulsed radiofrequency of the intercostal nerves in the treatment of chronic postsurgical thoracic pain. Pain physician. 2006; 9: 227-235.

23. Cruccu G, Aziz TZ, Garcia-Larrea L, et al. EFNS guidelines on neurostimulation therapy for neuropathic pain. Eur J Neurol. 2007; 14: 952-970.

24. Oosterhof J, Wilder-Smith OH, Oostendorp RA, et al. Different mechanisms for the shortterm effects of real versus sham transcutaneous electrical nerve stimulation (TENS) in patients with chronic pain: a pilot study. J Pain Palliat Care Pharmacother. 2012; 26: 5-12.

25. Philip CN, Candido KD, Joseph NJ, et al. Successful treatment of meralgia paresthetica with pulsed radiofrequency of the lateral femoral cutaneous nerve. Pain physician. 2009; 12: $881-885$.

26. Werner MU, Bischoff JM, Rathmell JP, et al. Pulsed radiofrequency in the treatment of persistent pain after inguinal herniotomy: a systematic review. Reg Anesth Pain Med. 2012; 37: 340-343. 


\section{Chapter 10}

Valorisation 


\section{Introduction}

The anterior cutaneous nerve entrapment syndrome is a frequently overlooked, and to many physicians, a largely unknown entity ${ }^{1-3}$. Over the last century, just a handful of physicians have reported on a limited number of aspects of this painful syndrome $e^{3-11}$. Most contributions focused on clinical presentation, diagnosis and basic treatment options. Although these papers undoubtedly had educational value, they did not lead to a permanently increased awareness among physicians. The present work highlights the ACNES incidence and prevalence in certain populations, and the socio-economic impact of a disease that causes pain. The cost due to medical consumption and loss of productivity as a consequence of having an unrecognised disease are investigated. The most important aspect of it all is to 'increase awareness' among general practitioners as well as a wide variety of medical specialists such as internists, gastroenterologists, emergency physicians, gynaecologists, urologists and paediatricians. We therefore chose to not only publish our work in surgical journals but also in journals for general practitioners, gastroenterologists and emergency medicine physicians. Our work was also presented on several national and international meetings of different specialties. Furthermore, information appeared in national newspapers and TV media over the last years whereas a website was launched containing information for both patient and doctor (www.buikpijn.nl).

\section{Socio-economic relevance}

As concluded in chapter 5 , in the year preceding the diagnosis ACNES patients consume on average $€ 5,400$ on health care costs and have an average productivity loss of $€ 13,800$. Based on a calculated 57:100.000 ACNES incidence rate (chapter 4), costs of illness can be approximated ${ }^{12}$. When extrapolated to the Dutch population $(2015,16.900 .000$ inhabitants), some 9.500 new ACNES patients may be identified yearly ${ }^{13}$. On the basis of these assumptions, total yearly costs associated with ACNES in The Netherlands can be estimated at $€ 182.4$ million ( $€ 51.3$ million direct medical costs and $€ 131.1$ million indirect non-medical costs), assuming that all newly diagnosed ACNES patients experience a similar burden of disease, which is not necessarily true. However, the calculated incidence rate (chapter 4) was also based on ACNES patients with severe complaints, since they all consulted an outpatient department or an emergency department for unacceptable pain ${ }^{12}$. If all these patients would undergo a diagnostic and treatment regimen as proposed in the present thesis ( $\pm € 2,800$ on average per patient for diagnosis and treatment), direct medical costs are calculated at $€ 26.6$ million per year. An indirect non-medical cost calculation cannot reliably be determined, but a maximum half a year treatment period (including waiting time prior to first evaluation as well as time periods between treatment steps) is reasonable. Assuming similar productivity losses as calculated in this thesis, early detection and treatment would lead to an estimated yearly €80-100 million cost reduction. Thus, it can safely be concluded that earlier recognition will substantially reduce both the suffering amongst individuals as well as reduce unnecessary direct and indirect societal costs. Indeed, 'Carnett cuts costs' ${ }^{\text {'14. }}$

\section{Relevance in the medical field}

The studies in this thesis are just based on incidence and prevalence in The Netherlands. It is reasonable to assume that ACNES is also common and underdiagnosed in the rest of the world. Over the last few years we have received requests for help (e-mail consultations or personal visits) from patients and physicians from other parts of the world (USA, United Kingdom, Sweden, Greece, South Africa, Germany, Belgium, Denmark). The vast majority of patients had learned about us through the internet or through physicians who had read our publications. In these patients a provisional diagnosis of ACNES was established. A common question was whether we knew a physician or pain specialist in their respective country who was knowledgeable and willing to treat this pain entity. We discovered that the number of physicians who treat ACNES worldwide is exceedingly limited. As a result, a handful of foreign patients (i.e. US and UK) came to the SolviMáx center for treatment.

The first few chapters focused on epidemiological aspects of the syndrome and provided more solid data compared to previous reports that were mostly based on personal experiences of individual physicians ${ }^{4,15-17}$. The results of the present thesis will aid in recognising the syndrome and allow for a standard inclusion of ACNES in the differential diagnosis of abdominal pain. In a hypothetical ideal situation, each physician is aware of the existence of the syndrome and recognises its characteristic symptoms 'at first sight'. If so, a long and frustrating (for both doctors and patients) diagnostic process is prevented whereas patients do get a tailored approach in an early phase of the illness. Apart from the enormous societal cost reduction as earlier discussed, a serious reduction in disease burden (both duration and severity of complaints) amongst ACNES patients 
can be achieved. Currently, some patients are labelled with psychosomatic disorders when the pain is not recognised as due to ACNES. As a consequence, these patients feel frustrated and ignored leading to uncertainty and depression.

A regimen of consecutive abdominal wall injections followed by surgery in resistant cases is the method of choice as demonstrated in a cohort of 139 ACNES patients ${ }^{18}$. In the present thesis, the efficacy of the anterior neurectomy is unequivocally demonstrated by a randomised controlled trial and by studying the long-term results of the technique. A novel second invasive treatment step ('posterior neurectomy') is added to the existing surgical protocol leading to improved overall results. As a consequence, some $90 \%$ of ACNES patients can now be treated successfully. Most of these patients are able to return to normal daily activities instead of living the life of a chronic pain patient.

\section{References}

1. Gallegos NC, Hobsley M. Abdominal wall pain: an alternative diagnosis The British journal of surgery. 1990; 77: 1167-1170.

2. Hershfield NB. The abdominal wall. A frequently overlooked source of abdominal pain J Clin Gastroenterol. 1992; 14: 199-202.

3. Srinivasan R, Greenbaum DS. Chronic abdominal wall pain: a frequently overlooked problem. Practical approach to diagnosis and management. Am J Gastroenterol. 2002; 97 824-830.

4. Carnett J. Intercostal neuralgia as a cause of abdominal pain and tenderness. Surg Gynecol Obstet. 1926; 42: 8.

5. Ranger I, Mehta M, Pennington M. Abdominal wall pain due to nerve entrapment. Practitioner. 1971; 206: 791-792.

6. Applegate WV. Abdominal cutaneous nerve entrapment syndrome. Surgery. 1972; 71: 118-124.

7. Doouss TW, Boas RA. The abdominal cutaneous nerve entrapment syndrome N Z Med J. 1975; 81: 473-475.

8. Thomson H, Francis DM. Abdominal-wall tenderness: A useful sign in the acute abdomen. Lancet. 1977; 2: 1053-1054.

9. Gallegos NC, Hobsley M. Recognition and treatment of abdominal wall pain. J R Soc Med 1989; 82: 343-344.

10. Roumen RM, Scheltinga MR. [Abdominal intercostal neuralgia: a forgotten cause of abdominal pain]. Ned Tijdschr Geneeskd. 2006; 150: 1909-1915.

11. Scheltinga MR, Boelens OB, Tjon ATWE, et al. Surgery for refractory anterior cutaneous nerve entrapment syndrome (ACNES) in children. J Pediatr Surg. 2011; 46: 699-703.

12. van Assen T, Brouns JAGM, Scheltinga MR, et al. Incidence of abdominal pain due to the anterior cutaneous nerve entrapment syndrome in an emergency department. Scand J Trauma Resusc Emerg Med. 2015.

13. Statistiek CBvd. Bevolkingsteller. [web page] [cited 2012; Available from: http://www.cbs. nl/nl-nl/menu/themas/bevolking/cijfers/extra/bevolkingsteller.htm.

14. Abdominal wall tenderness test: could Carnett cut costs? Lancet. 1991; 337: 1134

15. Applegate WV. Abdominal Cutaneous Nerve Entrapment Syndrome (ACNES): A Commonly Overlooked Cause of Abdominal Pain. Perm J. 2002; 6: 20-27. 
16. Hall PN, Lee AP. Rectus nerve entrapment causing abdominal pain. The British journal of surgery. 1988; 75: 917.

17. Thomson WH, Dawes RF, Carter SS. Abdominal wall tenderness: a useful sign in chronic abdominal pain. The British journal of surgery. 1991; 78: 223-225.

18. Boelens OB, Scheltinga MR, Houterman S, et al. Management of anterior cutaneous nerve entrapment syndrome in a cohort of 139 patients. Annals of surgery. 2011; 254: 1054-1058. 


\section{Chapter 11}

Samenvatting

(Dutch summary) 


\section{Samenvatting}

Het onderwerp 'buikwandpijnsyndromen', waaronder het 'anterior cutaneous nerve entrapment syndrome' (ACNES) heeft nooit de aandacht gekregen die het eigenlijk verdient. In de afgelopen eeuw is er toch af en toe vanuit verschillende disciplines en landen gepubliceerd over dit syndroom ${ }^{1-8}$. Helaas heeft dit beperkt aantal studies niet geleid tot een voldoende kennisniveau omtrent buikwandpijnsyndromen. Een enquête onder Nederlandse chirurgen $(n=425)$ en chirurgen in opleiding $(n=82,35 \%$ respons ratio) in 2006 toonde bijvoorbeeld aan dat $85 \%$ van de ondervraagden niet bekend was met dit pijnsyndroom. Van de chirurgen met $>10$ jaar ervaring gaf slechts $18 \%$ aan ooit geconfronteerd te zijn geweest met een buikwandpijnsyndroom zoals ACNES. Ook was slechts $9 \%$ van de chirurgen-in-opleiding bekend met de symptomen hiervan ${ }^{7}$. Deze percentages zijn onacceptabel laag, ook al omdat patiënten met deze klachten vaak worden verwezen naar een chirurg. Het vermoeden is dat kennis bij andere, niet chirurgische, specialismen mogelijk nog beperkter is.

ACNES is een typisch neuropatisch pijnsyndroom dat is geassocieerd met een hoog pijnniveau, waardoor de meeste patiënten medische hulp zullen zoeken. Als de geconsulteerde arts het syndroom niet herkend (gezien het bovenstaande niet ondenkbaar), worden vaak onnodige en kostbare diagnostische onderzoeken verricht op verzoek van huisartsen en medisch specialisten ${ }^{6,9}$. Eén studie toonde aan dat er niet minder dan 418 diagnostische procedures waren uitgevoerd bij 100 buikwandpijnpatienten ${ }^{10}$. Een ander onderzoek uit 1994 liet zien dat de work-up van een buikwandpijn-patiënt gemiddeld US\$ 680 kostte 5 . Een derde onderzoek uit 2001 toonde aan dat een bedrag van maar liefst US\$ 6727 per patiënt werd uitgegeven aan (uiteindelijk) niet bijdragend diagnostisch onderzoek en ziekenhuiskosten vóórdat de juiste buikwandpijn gerelateerde diagnose werd gesteld ${ }^{11}$. Deze onderzoeken tonen onafhankelijk van elkaar aan dat de economische belasting van diagnostische entiteiten, zoals uitgevoerd voor ACNES, substantieel is.

In de huidige medische praktijk met een bijna ongelimiteerde toegang tot diagnostische middelen, lijkt de rol van een gedegen lichamelijk onderzoek minder belangrijk te zijn geworden. Een buikwandpijnsyndroom zoals ACNES is echter juist een typisch voorbeeld van een klinisch syndroom, dat moet worden vastgesteld op basis van de bevindingen uit een gedegen anamnese en lichamelijk onderzoek. ACNES-patiënten hebben een karakteristiek verhaal van een continu scherpe of brandende pijn, die in bepaalde situaties of houdingen toeneemt ${ }^{12,13}$. Het gebied van de maximale pijn is normaliter zeer plaatselijk en bevindt zich binnen laterale grenzen van de musculus rectus abdominis. De pijn is vaak op een acuut moment ontstaan, na bijvoorbeeld een operatie, een zwangerschap, sportbeoefening of een onverwachte beweging. Echter, in ongeveer de helft van de patiënten kan de oorzaak niet goed achterhaald worden ${ }^{12}$. Specifieke houdingen en lichamelijke activiteit kunnen de klachten verder uitlokken. Ook kunnen symptomen die een orgaan-gerelateerd probleem doen vermoeden (misselijkheid, opgeblazen gevoel, obstipatie, etc.), voorkomen.

ACNES is dus bij uitstek een typisch klinisch syndroom, waarbij het lichamelijk onderzoek cruciaal is voor de diagnose. Er is sprake van een pijnlijk gebiedje in de buik met een klein maximaal pijnpunt $\left(<2 \mathrm{~cm}^{2}\right.$, 'top van een vinger') binnen de grenzen van de rectus abdominis spier. De pijn neemt toe op het moment dat de onderzoeker het maximale pijnpunt palpeert terwijl de patiënt de buikspieren aanspant (Carnett's test). Sensibiliteitsstoornissen rondom het pijnpunt zijn vaak aanwezig. De zogeheten 'pinch test', waarbij een kleine huidplooi ter hoogte van het pijnpunt tussen duim en wijsvinger wordt vastgepakt, veroorzaakt vaak een vervelende pijnsensatie vergeleken met de niet aangedane zijde. Als de diagnose ACNES waarschijnlijk wordt geacht, kan infiltratie van de buikwand met lidocaine of bupivacaïne worden overwogen om de diagnose te bevestigen. Een serie van 2 of 3 injecties (vaak met 1-2 weken tussenpozen) is blijvend effectief bij ongeveer $1 / 3^{\text {e }}$ van de ACNES-patienten ${ }^{12,13}$. De tweede behandelstap bij patiënten die niet blijvend gunstig reageren op injecties is een chirurgische neurectomie, die bij ongeveer $2 / 3^{e}$ van de overgebleven patiënten effectief is. Dit behandelprotocol heeft geleid tot een totaal succespercentage van ongeveer $80 \%$ bij 139 ACNES patiënten met een mediane follow up van 18 maanden ${ }^{12}$. Hoofdstuk 1 (chapter 1) geeft een meer gedetailleerde omschrijving van achtergrond, diagnose en behandeling van ACNES.

Het eerste hoofdthema van dit proefschrift is gericht op het bestuderen van de epidemiologie en de economische impact van ACNES. Patiënten waarbij een 'diagnose per exclusionem' zoals het prikkelbare darmsyndroom (PDS) werd vastgesteld of vermoed, lijden mogelijk aan een buikwandsyndroom zoals ACNES, hetgeen tot dan toe over het hoofd is gezien. Een PDS populatie is heterogeen en bevat een grote variëteit aan symptomen en klachten. Volgens de Rome III criteria is de diagnose PDS gebaseerd 
op een combinatie van klachten, zonder de aanwezigheid van structurele anatomische afwijkingen. Verrassend genoeg zijn er geen bevindingen uit het lichamelijk onderzoek opgenomen in de Rome III criteria. Volgens de definitie moeten PDS patiënten ten minste 3 dagen per maand in de afgelopen 3 maanden buikpijnklachten rapporteren. De diagnose PDS kan worden gesteld als er aan 2 of meer van de volgende criteria wordt voldaan. De buikpijnklachten ontstaan met een verandering in frequentie en/ of consistentie van de ontlasting, waarbij de klachten verminderen na stoelgang ${ }^{14}$. De hypothese in het huidige proefschrift is dat sommige ACNES patiënten mogelijk onjuist als PDS zijn gediagnosticeerd, aangezien PDS en ACNES overeenkomstige symptomen kunnen hebben. In hoofdstuk 2 (chapter 2) worden ontwikkeling en validatie van een vragenlijst beschreven, die in potentie onderscheid kan maken tussen patiënten met ACNES en PDS. Een simpele lijst van 18 vragen ( 0 - 18 punten) blijkt een sensitiviteit van 0.94 te hebben, gecombineerd met een specificiteit van 0.92 bij een afkapwaarde van 10 punten. Deze vragenlijst kan dus effectief beide patiëntengroepen onderscheiden.

De studie die wordt beschreven in hoofdstuk 3 (chapter 3) laat zien dat ACNESpatiënten inderdaad onterecht gediagnosticeerd kunnen worden met een functionele buikpijndiagnose zoals PDS. Vier Nederlandse huisartsen die niet van het bestaan van ACNES afwisten werden geselecteerd in deze cross-sectionele analyse. 535 patiënten ouder dan 18 jaar die in deze praktijken waren geregistreerd met een functionele buikpijndiagnose werden benaderd om de ontwikkelde 18 item vragenlijst in te vullen. Van de 167 symptomatische respondenten scoorden 18 individuen boven de vastgestelde afkapwaarde van 10 punten, en hadden dus mogelijk een buikwandpijnsyndroom. $\mathrm{Zij}$ ondergingen een diagnostische evaluatie bestaande uit anamnese en lichamelijk onderzoek om tot een definitieve diagnose te komen. De helft van deze patiënten $(n=9)$ bleek inderdaad een buikwandpijnsyndroom te hebben, en 6 hiervan voldeden aan de kenmerken van ACNES, hetgeen overeenkomt met een prevalentie van 3.6\% (95\% CI, 1.7-7.6) binnen de onderzochte prikkelbare-darm-populatie. Gebaseerd op de geschatte prevalentie van PDS (Nederlands Huisartsen Genootschap), zoekt in Nederland ongeveer een half miljoen mensen met PDS medische hulp voor hun buikpijnklachten. Op basis van deze aanname is het mogelijk dat zo'n 20 duizend individuen, bij wie eigenlijk de diagnose PDS is gesteld, in werkelijkheid lijden aan ACNES (95\% CI, 9.500 - 42.000). De totale aantallen ACNES-patiënten in huisartsenpraktijken in het algemeen zijn mogelijkerwijs nog hoger, aangezien bij deze studie alleen is gekeken binnen de PDS populatie en niet bij andere (buik)pijn-patiënten. Resultaten in deze studie kunnen een eyeopener voor huisartsen zijn.

Het doel van de observationele studie van hoofdstuk 4 (chapter 4) is om een indruk te krijgen van de incidentie van ACNES op de spoedeisende hulp (SEH) van een Nederlands opleidingsziekenhuis. Samen met de verkregen incidentiecijfers van patiënten die werden geëvalueerd op de chirurgische polikliniek kan zo een algemene incidentie worden bepaald. Eerst werden patiënten die de SEH van Máxima Medisch Centrum (MMC) in 2011 o 2012 bezochten met de zoektermen 'ACNES' 'intercostaalneuralgie' en 'buikwandpijn' geïdentificeerd. Dossiers van potentiële patiënten die binnen het adherentiegebied van het ziekenhuis woonden, werden beoordeeld op de aanwezigheid van ACNES aan de hand van bestaande en geaccepteerde criteria van anamnese en lichamelijk onderzoek. De ziekte-incidentie kon vervolgens worden bepaald aan de hand van het eigen MMC-adherentiegebied met de daarbij behorende patiëntaantallen. De incidentie van ACNES-patiënten die in het daaropvolgende jaar (2013) via de polikliniek werden gediagnosticeerd, werd op een gelijke wijze berekend. Gedurende de studieperiode van 2 jaar, werd bij 88 patiënten binnen het adherentiegebied van MMC na een SEH-bezoek de diagnose ACNES gesteld, hetgeen neerkomt op een jaarincidentie van 22/100.000. Dit betekent dat ongeveer 1 ACNES diagnose wordt gesteld per 50 patiënten die zich met acute buikpijn melden op de SEH. Hiernaast kon over 2013 een 35/100.000 jaarincidentie worden bepaald van ACNES patiënten die via de polikliniek werden geëvalueerd. Het combineren van deze twee incidentiematen leidt tot een totale ACNES incidentie van 1:1.800 per jaar in de algemene populatie.

Eerdere studies hebben de hoge diagnostische kosten bij ACNES-patiënten al onder de aandacht gebracht. Deze onderzoeken dateren echter van decennia geleden en zijn uitgevoerd in buitenlandse gezondheidssystemen. Een analyse van directe medische en indirecte productiviteitskosten in het jaar voorafgaande aan de diagnose in een ACNESpopulatie wordt beschreven in hoofdstuk 5 (chapter 5). Patiënten die van buiten he adherentiegebied van MMC kwamen en waarbij de diagnose ACNES in MMC werd gesteld tussen september 2012 en augustus 2014 waren geschikt voor studiedeelname. De onderzoekspersonen werden verzocht een medische consumptie vragenlijst (iMCQ) en een productiviteitsvragenlijst (iPCQ) in te vullen. De aantallen uit de zorgconsumptie vragenlijst werden vermenigvuldigd met de kostprijs per eenheid, om zo de diagnostische 
en therapeutische kosten te kunnen berekenen. Productiviteitsverliezen werden gecalculeerd door kosten van absentie, productiviteitsverlies zonder absentie en verlies van onbetaalde arbeid bij elkaar op te tellen. 94 patiënten voldeden aan de studiecriteria (vrouw $\mathrm{n}=74,79 \%$; gemiddelde leeftijd 41 jaar, SD 17). Totaal berekende kosten van zorgconsumptie kwamen neer op gemiddeld $€ 5,400$ per patiënt. Extrapolatie naar de totale klachtenduur voordat de diagnose werd gesteld (mediaan ongeveer 4 jaar), leidde tot een gemiddelde van $€ 16,600$ aan directe zorgkosten per patiënt. De productiviteitsverliezen werden berekend op $€ 13,800$ per patiënt in het jaar vóórdat de diagnose werd gesteld. Deze calculaties leiden tot een totaal van €19,200 aan kosten in het jaar vóór de diagnose ACNES per patiënt. Concluderend kan gesteld worden dat ACNES-patiënten een hoge zorgconsumptie en veel productiviteitsverliezen vertonen in het jaar voorafgaand aan de diagnose. Eerdere herkenning van dit syndroom zal deze kosten waarschijnlijk sterk verminderen.

Het tweede hoofdonderwerp van dit proefschrift betreft de chirurgische behandeling van ACNES. Hoewel ongeveer $1 / 3^{e}$ van de ACNES-patiënten op de lange termijn succesvol met behulp van lokale injecties kan worden behandeld, ervaren de meeste andere patiënten geen blijvend resultaat van deze initiële behandelstap. Deze restgroep wordt een anterieure neurectomie aangeboden, hetgeen in een eerdere retrospectieve analyse succesvol is gebleken bij ongeveer 2 van de 3 geopereerde patiënten ${ }^{19}$. De goede resultaten van een dergelijke chirurgische interventie bij deze patiëntenpopulatie was echter nog niet aangetoond in een gerandomiseerd gecontroleerd onderzoek. In hoofdstuk 6 (chapter 6) wordt een onderzoek beschreven waarin volwassen ACNES-patiënten, die onvoldoende pijnvrij zijn na injecties, worden gerandomiseerd voor een anterieure neurectomie dan wel een 'schijnoperatie'. Uiteraard was deze studie door de lokale Medisch Ethische Commissie goedgekeurd. Zowel de patiënt als de hoofdonderzoeker waren tot 6 weken na de operatie niet op de hoogte van de aard van de ingreep. Het pijnniveau werd genoteerd vóór de ingreep, direct na de ingreep en 6 weken postoperatief. Een $>50 \%$ pijnreductie (VAS, visuele analoge schaal) en/of een reductie van minimaal 2 punten (VRS, verbale pijnschaal) werd beschouwd als 'succes'. 44 patiënten participeerden in de periode tussen augustus 2008 en december 2010. In de neurectomiegroep reageerden 16 van de 22 patiënten succesvol op de ingreep. In de schijnoperatiegroep daarentegen reageerden slechts 4 patiënten met succes $(\mathrm{p}=0.001)$. Deze studie toont aan dat de pijn bij de meeste ACNES patiënten wordt veroorzaakt door de eindtakken van de intercostaal zenuwen en dat een neurectomie hierbij veelal goed kan helpen.
Een anterieure neurectomie lijkt een redelijk succesvolle behandeling bij ACNESpatiënten, maar de studies die dit aantonen zijn relatief klein met een korte follow-up. Het doel van hoofdstuk 7 (chapter 7) is om de lange-termijn-succespercentages te bepalen van chirurgie in een grote ACNES-populatie. Alle patiënten die een anterieure neurectomie ondergingen tussen januari 2004 en februari 2012 werden onderzocht. Pijnscores vóór en na de operatie en op het moment van ondervragen werden verkregen middels een 10-punts pijnscore (PI-NRS) en een 6-punts verbale pijnscore (VRS). Succes werd gedefinieerd als $\geq 50 \%$ pijnreductie op de PI-NRS en/of minstens 2 punten reductie op de VRS. Bij 154 individuen werden 181 neurectomieën uitgevoerd en derhalve geanalyseerd. Op de korte termijn (1-3 maanden postoperatief) werd bij 70\% een succesvolle respons vastgesteld. Na een mediane follow up van 32 maanden (3-93 maanden) kon een succesvolle respons bij $61 \%$ worden vastgesteld. Bij $16 \%(n=20)$ werd een recidief ACNES (klachten op exact dezelfde plaats) waargenomen. Concluderend kan worden gesteld dat een operatie bij ACNES-patiënten die onvoldoende reageren op injecties ook op de lange termijn bij ongeveer 2 van de 3 patiënten succesvol blijft.

Hoewel het behandelprotocol, bestaande uit lokale infiltraties gevolgd door een anterieure neurectomie, op de lange termijn succesvol is bij de meeste patiënten, zijn de opties voor het resterende kwart van de ACNES patiënten, die uiteindelijk niet goed reageren, nooit onderzocht. In hoofdstuk 8 (chapter 8) wordt de effectiviteit van alternatieve chirurgische opties na een niet succesvolle eerste operatie onderzocht. Dit zijn enerzijds patiënten die überhaupt nog nooit succes van behandeling hebben ervaren, en anderzijds patiënten die aanvankelijk wel een succesvolle respons op operatie hadden, maar bij wie de ACNES recidiveerde. Bij een tweede operatie zijn er twee opties, een secundaire anterieure neurectomie (re-exploratie) of een niet nog nooit eerder beschreven techniek, de zogenaamde 'posterieure neurectomie'. Volwassen patiënten met recidiverende klachten of een initieel niet succesvolle respons, werden voor deze studie geselecteerd uit een database van 181 chirurgische procedures die tussen 2004 en 2012 in het Máxima Medisch Centrum zijn uitgevoerd. De pijnniveaus rondom de re-exploratie of posterieure neurectomie werden opnieuw middels de PI-NRS en VRS bepaald, waarbij postoperatief succes werd gedefinieerd als minstens $50 \%$ pijnreductie op de PI-NRS en/of minimaal 2 punten reductie op de VRS. 51 patiënten die na een eerdere neurectomie onacceptabele pijnniveaus rapporteerden, en 20 patiënten die recidief klachten ontwikkelden, konden voor deze studie worden geselecteerd. Van 
deze 71 patiënten ondergingen 41 uiteindelijk opnieuw een chirurgische ingreep (reexploratie, $n=10$; posterieure neurectomie, $n=31$ ). Na een mediane follow up van 25 maanden, bleek een tweede operatie bij 66\% van de patiënten succesvol. Hierbij toonden patiënten met recidief ACNES betere resultaten (14/15) dan de patiënten bij wie al direct na de eerste operatie pijnklachten onverminderd anwezig bleven $(13 / 26, \mathrm{p}=0.01)$. Op basis van deze studie kan worden vastgesteld dat een tweede operatie (re-exploratie of posterieure neurectomie) de moeite waard kan zijn bij ACNES-patiënten, die recidief of persisterende pijnklachten ervaren na een eerste operatie.

\section{Conclusies van dit proefschrift}

1. Een in dit onderzoek ontwikkelde 18-item vragenlijst kan een 'buikwandpijnsyndroom' identificeren bij mensen waarbij in een eerder stadium onterecht de diagnose 'functionele buikpijn' is gesteld (sensitiviteit 0.92, specificiteit 0.94).

2. In huisartsenpraktijken blijkt een klinisch relevant deel van patiënten met 'functionele buikpijn' in werkelijkheid aan ACNES te lijden (prevalentie 3.6\%, CI $1.7-7.6 \%)$.

3. De incidentie van acute ACNES-patiënten op een spoedeisende hulp (SEH) van een regionaal opleidingsziekenhuis is $22 / 100.000$ per jaar. Dit betekent dat ongeveer 2\% van de acute SEH buikpijnpatiënten ACNES heeft. De algemene jaarincidentie van ACNES is vastgesteld op 1:1.800, hetgeen aangeeft dat er in Nederland jaarlijks ongeveer 9.500 nieuwe ACNES-patiënten bijkomen.

4. Patiënten met ACNES hebben een forse zorgconsumptie in het jaar voorafgaand aan hun diagnose ( $€ 5,400$ per patiënt). Extrapolatie van deze kosten op basis van het aantal jaren dat patiënten gemiddeld klachten hebben vóórdat de juiste diagnose wordt gesteld, leidt tot een bedrag van $€ 16,600$ aan directe medische kosten. Daarnaast blijken in het jaar voorafgaand aan de diagnose de indirecte kosten gemiddeld $€ 13,800$ te zijn. Derhalve wordt per patiënt in totaal $€ 19,200$ aan direct en indirecte kosten gemaakt in het jaar voorafgaand aan de diagnose.

5. Een neurectomie van anterieure eindtakken van de intercostaalzenuw leidt to een significante pijnreductie bij ACNES-patiënten die niet succesvol konden worden behandeld met injectietherapie. Met deze ingreep kan een korte termijn behandelsucces van $70 \%$, en een lange termijn succes van $61 \%$ worden bereikt.

6. Een secundaire chirurgische ingreep zoals een re-exploratie of een posterieure neurectomie is succesvol bij ongeveer $2 / 3^{e}$ van de ACNES-patiënten bij wie de pijn na een eerdere anterieure neurectomie persisteerde of recidiveerde. 


\section{Toekomstperspectief}

Algemene informatie rondom incidentie, prevalentie, diagnose en behandeling van ACNES is schaars. De inspanningen die tot nu toe in het SolviMáx Expertise Centrum zijn geleverd, hebben mede als doel om hiaten in deze basiskennis op te vullen. Een toenemend aantal verwezen patiënten voorziet ons van een unieke mogelijkheid om nieuwe onderzoeksvragen te kunnen beantwoorden. Een praktische aanpak binnen SolviMáx heeft ertoe geleid dat een simpel uitvoerbaar behandelprotocol is ontwikkeld, dat voor de meeste ACNES-patiënten een goede uitkomst biedt. Echter, er is nog steeds een kleine minderheid binnen deze buikwandpijnpopulatie, die ondanks alle behandelingsopties die tot nu toe ontwikkeld zijn, nog steeds onvoldoende baat hebben.

Eén belangrijk gebied van toekomstig onderzoek betreft dus de groep patiënten die niet goed reageert op het bestaande behandelprotocol. Belangrijke vragen als: 'hoe reageerden deze patiënten op de initiële injectietherapie?', 'hoe lang hadden zij al klachten?', 'welke klinische kenmerken bij lichamelijk onderzoek waren aanwezig?' moeten nog worden beantwoord. Door het inzichtelijk maken van verschillen in patronen tussen hen die goed reageren en patiënten die niet succesvol zijn behandeld, kunnen mogelijk prognostische factoren worden blootgelegd. Misschien kunnen op basis hiervan verschillende ACNES subtypes worden gedefinieerd. Kinderen vormen mogelijk al zo'n subtype ${ }^{8}$. De etiologie bij kinderen lijkt veelal mechanisch van aard, wat leidt tot symptomen die enkel tot de anterieure tak van de intercostaalzenuw herleid kunnen worden. In de ideale situatie zullen deze prognostische factoren leiden tot een individueel patiënt-specifiek behandelplan. Ook zal, gezien het feit dat veel patiënten ook (pseudo) viscerale klachten aangeven die na behandeling verdwijnen, de rol van segmentale relaties moeten worden onderzocht en nader verduidelijkt.

Deze thesis heeft zich ook gericht op de last die ACNES met zich meebrengt in maatschappelijke en economische zin. Onze studies toonden het onvermogen van patiënten aan om gewone, dagelijkse activiteiten te volbrengen, gezien de hoge absentiecijfers en productiviteitsverlies in de arbeidssituatie. Het is zeer aannemelijk dat de vaak langdurige zoektocht om tot de juiste diagnose te komen een serieuze impact heeft op de kwaliteit van leven. Voor een beter inzicht en begrip zou de rol van deze psychosociale factoren bij ACNES-patiënten intensiever bestudeerd moeten worden.
Toekomstig onderzoek zou zich moeten toeleggen op een complete evaluatie van de gewijzigde gezondheidsstatus van deze patiëntenpopulatie.

Een kwaliteit van een niet-academisch centrum zoals MMC is het pragmatisch aanpakken van medische vraagstukken. Zo is een nagenoeg vergeten buikwandpijnsyndroom zonder een gestructureerde diagnostische aanpak en behandelprotocol '(her)ontdekt', waarvoor een wetenschappelijk programma werd opgezet, dat zich heeft gefocust op snelle en betere herkenning, diagnostiek en behandeling. Verschillende bijdragen in toonaangevende medische tijdschriften hebben geleid tot een stapsgewijs behandelprotocol. Dit protocol blijkt bovendien te leiden tot betere resultaten dan gerapporteerd in vergelijkbare andere chronische perifere neuropathische pijnpopulaties $^{20,21}$. Al deze inspanningen hebben in een relatief kort tijdbestek plaatsgevonden. De keerzijde van deze praktische aanpak is echter wel dat een aantal fundamentele vraagstukken nog niet opgehelderd zijn. Toekomstig onderzoek zou zich, bij voorkeur met een academische partner, meer moeten richten op de anatomie van de aangedane zenuwstructuren en pathofysiologie van dit syndroom. Deze oproep lijkt in te druisen tegen een logische, stapsgewijze onderzoeksaanpak, maar het kan wel nieuwe inzichten geven die bijvoorbeeld een duidelijke classificatie mogelijk maken met een daarbij behorende specifieke behandeling. Een beter begrip van de neuroanatomie kan bovendien bijdragen aan alternatieve (chirurgische) behandeltechnieken. Zoals ook in dit proefschrift duidelijk werd, hadden sommige patiënten baat bij een nieuwe chirurgische techniek, de zogeheten 'posterieure neurectomie'. Bij deze benadering worden de intercostaal-zenuwtakjes geïdentificeerd ter hoogte van de achterste rectusfascie. Een succesvolle respons in sommige patiënten suggereert dat de zenuwbeknelling ook ter hoogte van de achterste rectusfascie kan vóórkomen. Deze redenering volgend zou een posterieure neurectomie, als eerste chirurgische stap, een betere behandeloptie kunnen zijn dan de anterieure neurectomie, angezien de zenuw op een proximaler traject wordt doorgenomen. Weer een stap verder zou een neurectomie op een nog proximaler niveau zijn, bijvoorbeeld via een laparoscopische benadering. Ook andere, niet chirurgische therapeutische handelingen op een proximaler niveau zouden een optie kunnen zijn. Deze alternatieve behandelopties zouden in de nabije toekomst verder onderzocht kunnen worden. 
Vanuit onze heelkundige visie is het begrijpelijk dat de hierboven beschreven toekomstperspectieven veelal vanuit een chirurgisch oogpunt worden beschreven. Er liggen echter ook behandelopties buiten het chirurgische spectrum. Medicatie kan bij een deel van de ACNES-patiënten een positief effect hebben, maar goed opgezette studies ontbreken op dit moment ${ }^{20,21}$. Andere therapeutische interventies zoals ruggenmerg of dorsale ganglion neurostimulatie-therapie, paravertebrale blokkades en manuele therapie hebben mogelijk ook toegevoegde waarde ${ }^{22-26}$. In onze praktijk worden deze behandelopties vaak pas benut als de chirurgische opties falen, doch het lijkt zinvol om de waarde van deze behandelingen als eerste behandelstap nader te onderzoeken.

\section{References}

1. Carnett J. Intercostal neuralgia as a cause of abdominal pain and tenderness. Surg Gynecol Obstet. 1926; $42: 8$.

2. Ranger I, Mehta M, Pennington M. Abdominal wall pain due to nerve entrapment. Practitioner. 1971; 206: 791-792.

3. Thomson H, Francis DM. Abdominal-wall tenderness: A useful sign in the acute abdomen Lancet. 1977; 2: 1053-1054.

4. Gallegos NC, Hobsley M. Recognition and treatment of abdominal wall pain. J R Soc Med. 1989; 82: 343-344.

5. Greenbaum DS, Greenbaum RB, Joseph JG, et al. Chronic abdominal wall pain. Diagnostic validity and costs. Dig Dis Sci. 1994; 39: 1935-1941.

6. Applegate WV. Abdominal Cutaneous Nerve Entrapment Syndrome (ACNES): A Commonly Overlooked Cause of Abdominal Pain. Perm J. 2002; 6: 20-27.

7. Roumen RM, Scheltinga MR. [Abdominal intercostal neuralgia: a forgotten cause of abdominal pain]. Ned Tijdschr Geneeskd. 2006; 150: 1909-1915.

8. Scheltinga MR, Boelens OB, Tjon ATWE, et al. Surgery for refractory anterior cutaneous nerve entrapment syndrome (ACNES) in children. J Pediatr Surg. 2011; 46: 699-703.

9. Srinivasan R, Greenbaum DS. Chronic abdominal wall pain: a frequently overlooked problem. Practical approach to diagnosis and management. Am J Gastroenterol. 2002; 97: $824-830$.

10. Hershfield NB. The abdominal wall. A frequently overlooked source of abdominal pain J Clin Gastroenterol. 1992; 14: 199-202.

11. Thompson C, Goodman R, Rowe WA. Abdominal wall syndrome, a costly diagnosis of exclusion. Gastroenterol. 2001; 120: A637.

12. Boelens OB, Scheltinga MR, Houterman S, et al. Management of anterior cutaneous nerve entrapment syndrome in a cohort of 139 patients. Ann Surg. 2011; 254: 1054-1058.

13. Boelens OB, Scheltinga MR, Houterman S, et al. Randomized clinical trial of trigger point infiltration with lidocaine to diagnose anterior cutaneous nerve entrapment syndrome. Br J Surg. 2013; 100: 217-221.

14. Drossman M. Rome criteria. [Web page] 2011; Available from: http://www.romecriteria. org/.

15. Drossman DA, Whitehead WE, Camilleri M. Irritable bowel syndrome: a technical review for practice guideline development. Gastroenterol. 1997; 112: 2120-2137. 
16. Statistiek CBvd. Bevolkingsteller. [web page] [cited 2012; Available from: http://www.cbs. nl/nl-nl/menu/themas/bevolking/cijfers/extra/bevolkingsteller.htm.

17. Talley NJ, Weaver AL, Zinsmeister AR, et al. Onset and disappearance of gastrointestinal symptoms and functional gastrointestinal disorders. Am J Epidemiol. 1992; 136: 165-177.

18. Van der Velden J. Een nationale studie naar ziekten en verrichtingen in de huisartspraktijk. Basisrapport; Morbiditeit in de huisartspraktijk. 1991.

19. Applegate W. Abdominal Cutaneous Nerve Entrapment syndrome (ACNES): A Commonly Overlooked Cause Of Abdominal Pain. Perm J. 2002; 6: 20-27.

20. Attal N, Cruccu G, Baron R, et al. EFNS guidelines on the pharmacological treatment of neuropathic pain: 2010 revision. Eur J Neurol. 2010; 17: 1113-e1188.

21. Finnerup NB, Otto M, McQuay HJ, et al. Algorithm for neuropathic pain treatment: an evidence based proposal. Pain. 2005; 118: 289-305.

22. Cohen SP, Sireci A, Wu CL, et al. Pulsed radiofrequency of the dorsal root ganglia is superior to pharmacotherapy or pulsed radiofrequency of the intercostal nerves in the treatment of chronic postsurgical thoracic pain. Pain physician. 2006; 9: 227-235.

23. Cruccu G, Aziz TZ, Garcia-Larrea L, et al. EFNS guidelines on neurostimulation therapy for neuropathic pain. Eur J Neurol. 2007; 14: 952-970.

24. Oosterhof J, Wilder-Smith OH, Oostendorp RA, et al. Different mechanisms for the shortterm effects of real versus sham transcutaneous electrical nerve stimulation (TENS) in patients with chronic pain: a pilot study. J Pain Palliat Care Pharmacother. 2012; 26: 5-12.

25. Philip CN, Candido KD, Joseph NJ, et al. Successful treatment of meralgia paresthetica with pulsed radiofrequency of the lateral femoral cutaneous nerve. Pain physician. 2009; 12: $881-885$.

26. Werner MU, Bischoff JM, Rathmell JP, et al. Pulsed radiofrequency in the treatment of persistent pain after inguinal herniotomy: a systematic review. Reg Anesth Pain Med. 2012; 37: 340-343. 
Dankwoord

List of Publications

Curriculum vitae 


\section{Dankwoord}

De weg naar de totstandkoming van dit proefschrift is niet beperkt tot de jaren dat ik me actief heb ingezet voor de studies die dit boek bevatten. Enkele basisnormen en waarden zijn mij letterlijk met de paplepel ingegoten en deze hebben ertoe bijgedragen dat ik deze promotie in de huidige vorm heb kunnen afronden. Discipline, eerlijkheid en bescheidenheid zijn enkele eigenschappen die snel bij mij komen bovendrijven. Ik ben hier mijn ouders, Henk en Betsy van Assen, enorm dankbaar voor. Mijn broers Jan en Anton hebben hier sterk ook aan bijgedragen, waarvoor dank!

Ik had 4 jaar geleden niet kunnen bedenken dat ik auteur zou zijn van het boek dat nu voor u open ligt. Zonder de vele patiënten die het vertrouwen in mij hebben gesteld door te participeren in de onderzoeken die dit proefschrift bevat, had dit nooit gerealiseerd kunnen worden. Alleen met jullie deelname is het voor mij mogelijk geweest om kleine stukjes van die puzzel die ACNES heet op te lossen. Opnieuw is mij duidelijk geworden dat jullie de bron zijn van nieuwe informatie en de toegang zijn tot oplossingen.

Prof. dr. G.L. Beets en prof. dr. M. van Kleef, promotores, beste heren, bedankt voor het enthousiasme, de open houding en de goede samenwerking die het mogelijk hebben gemaakt om de afronding van dit proefschrift uiteindelijk in zo'n kort tijdsbestek te kunnen realiseren. Ik besef me dat mijn strakke tijdlijn en uw drukke agenda’s soms hebben kunnen conflicteren, maar dit heb ik zelf gelukkig nooit zo ervaren. Altijd kon ik rekenen op scherpe analyses en terechte vragen die hebben bijgedragen aan de kwaliteit van dit proefschrift.

Dr. R.M.H. Roumen, copromotor, beste Rudi, jij hebt mij 4 jaar geleden meegezogen in de wereld die ACNES heet. Jij zag iemand die je in staat achtte een bijdrage te kunnen leveren in het ACNES onderzoek. Ik zag en zie iemand die bevlogen is alles wat hij doet, gecombineerd met een flinke dosis overtuigingskracht. Dit geldt voor zowel de patiëntenzorg als het onderzoekswerk. Vanaf het eerste moment heb jij mij gegrepen met je enthousiasme en zie waar het toe geleid heeft! Je bent echt een 'wandelende ideeënbus' en hebt de capaciteit om in 5 minuten een heel proefschrift bij elkaar te filosoferen. Toch zal dit niet zijn wat me het meest is bijgebleven in de afgelopen jaren. Je volledige begrip en grenzeloze steun die jij, en ook Marc, hebt gegeven toen ik had besloten niet verder te gaan binnen de chirurgie, heeft diepe indruk op mij gemaakt. Je hebt het vermogen getoond om over je eigen schaduw heen te kijken en je bent je altijd vol voor mijn carrière blijven inzetten. Mijn persoonlijke keuzes heb je hierin boven de belangen van dit proefschrift gesteld. Als mens en als professional waardeer ik je daarom enorm. We zullen beiden meer en meer onze eigen weg inslaan, maar ik weet dat je deur altijd voor mij openstaat en dat is andersom niet anders.

Dr. M.R.M. Scheltinga, copromotor. Beste Marc, alias 'koning manuscriptrevisie, ik ken niemand die zo snel teksten reviseert als jij. Het blijft een raadsel hoe je dit voor elkaar krijgt en dit is daarom ook een terugkerend onderwerp van gesprek onder de onderzoekers van de afdeling chirurgie in het Máxima Medisch Centrum. Je snelle werkwijze, doortastende kritieken en nooit aflatende goede zin hebben mij ontzettend geholpen met het afronden van de verschillende studies. Daarnaast is onze gezamenlijke liefde voor het wielrennen en aangename afwisseling geweest op de ACNES problematiek. Bedankt voor het zijn van de katalysator van mijn proefschrift, 'I hope I kept you busy enough...'

Alle co-auteurs, dr. O.B. Boelens, dr. P.V. van Eerten, dr. C. Perquin, drs. J.Th. Kamphuis, drs. C.A.M. Bouwmans, drs. J.W. de Jager-Kievit, drs. J.A. Brouns, bedankt voor de samenwerking tijdens de verschillende onderzoeksprojecten. In het bijzonder Oliver, bedankt dat ik het stokje van je heb kunnen overnemen.

Prof. dr. Prof. dr. N.D. Bouvy, Prof. dr. F.J.P.M. Huygen, Prof. dr. J.F.M. Metsemakers, Prof. dr. J.J. van Overbeeke en Prof. dr. A.J.P.M. Smout, leden van de manuscriptcommissie. Dank voor het beoordelen van mijn proefschrift.

Collega's van de afdelingen waar ik in de afgelopen jaren werkzaam ben geweest in het Máxima Medisch Centrum. Jullie interesse, begrip, ideeën en opbeurende woorden zijn van onschatbare waarde geweest. Binnen de afdeling chirurgie heb ik me altijd welkom en gewaardeerd gevoeld, ondanks dat ik niet in de kliniek werkzaam was. Op de spoedeisende hulp heb ik de kans gekregen om mijn klinische vaardigheden verder te ontwikkelen, maar ook heb ik hier de vrijheid gekregen om het onderzoek prioriteit te geven op momenten dat dit nodig was. Binnen het escalatieteam kon ik altijd wel terecht een luisterend oor of een goed advies. Maar ook vond ik hier welkome ontspanning 
tussen de onderzoeks-werkzaamheden door. Op de afdeling sportgeneeskunde is de afronding van dit proefschrift met veel interesse en belangstelling gevolgd. Ook hier heb ik de vrijheid gekregen om 'tussen de bedrijven door' de nodige zaken af te handelen.

Medewerkers van het secretariaat, polikliniek en drukkerij, dank voor jullie engelengeduld, ondersteuning en nooit aflatende inzet. Els en Mijke van de SolviMáx polikliniek, dank voor jullie hulp bij het verspreiden en verzamelen van het vele onderzoeksgerelateerde papierwerk. Tommy van de drukkerij, geen deadline was jou te veel, altijd stonden jij en je team klaar om op tijd een opdracht af te krijgen.

Familie, bedankt voor jullie interesse in mijn 'proza'. Deze betrokkenheid doet een mens goed, vooral op momenten dat dingen niet lopen zoals je ze gepland hebt. Van mijn 95-jarige opa tot 'de melkboer' op familiedagen, allemaal hebben jullie op een eigen manier jullie bijdrage geleverd die mij de motivatie hebben gegeven om dit project af te ronden.

Vrienden, bij jullie kon ik altijd mijn frustraties uiten en de 'overwinningen' vieren. Van Thijs, Jasper en Pim, mijn maten van het eerste uur uit Epe, tot aan mijn Maastrichtse vrienden van O.H.D. Bonobo, allemaal hebben jullie je aandeel geleverd. De nodige vrije tijd heb ik in jullie aanwezigheid doorgebracht. Deze afleiding en relativering die jullie konden brengen was soms hoognodig. Maar het is ook fijn om te weten dat jullie er voor me zijn in tijden van tegenslag.

Hugo Fokkenrood, goede vriend en paranimf, wie van ons had kunnen vermoeden dat we op dezelfde dag ons proefschrift mochten verdedigen toen we vier jaar geleden beiden het avontuur aan gingen dat promoveren heet? Je staat altijd klaar met scherpe analyses en je brengt me soms weer met beide benen op de grond met je ontwapenende rationalisme. Je bent een doorzetter zoals ik er maar weinig ken, maar gelukkig zie jij net als ik ook het belang om de zaken soms ook op een luchtige manier te kunnen evalueren. Het maakt me trots ook jouw paranimf te mogen zijn.

Anton van Assen, broer, paranimf en goede vriend. Je intelligente analyses, bijzondere kijk op zaken gecombineerd met een gortdroog gevoel voor humor hebben mij zowel afleiding als nieuwe inzichten gegeven. Ik heb altijd op je kunnen rekenen en vertrouwen, daarom is het vanzelfsprekend voor mij dat je mij rugdekking zal geven tijdens mijn verdediging. Samen hebben we al veel meegemaakt en altijd zijn we er samen goed uitgekomen. Laten we deze gebeurtenis dan ook maar met een goed gevolg afsluiten!

Lieve, lieve Inge. Twijfels, overpeinzingen en onzekerheden. Op momenten kostte het je veel tijd om het schild dat ik had opgebouwd af te pellen, maar je hebt altijd het geduld bewaard om tot de kern te komen bij mij. Dan bleek keer op keer dat even het hart luchten verbluffend goed werkt. Ook de rondjes samen op de racefiets in het Brabantse landschap hebben mij geholpen om het hoofd leeg te maken. De inzichten die je mij heb gegeven vanuit een compleet andere invalshoek hebben bijgedragen aan dit proefschrift. Samen hebben wij al een mooie geschiedenis geschreven, en samen zullen we het boek van onze toekomst hoofdstuk voor hoofdstuk verder vormgeven. Ik kijk er naar uit 


\section{List of publications}

1. van Assen T, Brouns JA, Scheltinga MR, Roumen RM. Incidence of abdominal pain due to the anterior cutaneous nerve entrapment syndrome in an emergency department. Scand J Trauma Resusc Emerg Med. 2015 Feb 8;23(1):19. doi: 10.1186/s13049-015-0096-0. PMID: 25887961

2. van Assen T, Boelens OB, van Eerten PV, Perquin C, Scheltinga MR, Roumen RM Long-term success rates after an anterior neurectomy in patients with an abdominal cutaneous nerve entrapment syndrome. Surgery. 2015 Jan;157(1):137-43. doi: 10.1016/j. surg.2014.05.022. Epub 2014 Oct 14. PMID: 25444218

3. van Assen T, Boelens OB, van Eerten PV, Scheltinga MR, Roumen RM. Surgical options after a failed neurectomy in anterior cutaneous nerve entrapment syndrome. World J Surg. 2014 Dec;38(12):3105-11. doi: 10.1007/s00268-014-2737-2. PMID: 25189442

4. van Assen T, de Jager-Kievit JW, Scheltinga MR, Roumen RM. Chronic abdominal wall pain misdiagnosed as functional abdominal pain. J Am Board Fam Med. 2013 NovDec;26(6):738-44. doi: 10.3122/jabfm.2013.06.130115. PMID: 24204070

5. van Assen T, Boelens OB, Kamphuis JT, Scheltinga MR, Roumen RM. Construction and validation of a questionnaire distinguishing a chronic abdominal wall pain syndrome from irritable bowel syndrome. Frontline Gastroenterol. 2012 Oct;3(4):288-294. Epub 2012 Jul 26. PMID: 23914291

6. Boelens OB, van Assen T, Houterman S, Scheltinga MR, Roumen RM. A double-blind, randomized, controlled trial on surgery for chronic abdominal pain due to anterior cutaneous nerve entrapment syndrome. Ann Surg. 2013 May;257(5):845-9. doi: 10.1097/ SLA.0b013e318285f930. PMID: 23470571 


\section{Curriculum vitae}

The author of this thesis, Tijmen van Assen was born on the 3rd of March 1986 in Epe, a village situated in the northeastern part of Gelderland, The Netherlands. He grew up and graduated from high school in Epe.

After graduation at the 'Regionale Scholen-gemeenschap Noord Oost Veluwe', he finished in 3 years the bachelor Technical Medicine in Enschede. After this bachelor Tijmen moves to Maastricht to study the 4-year masters degree in Medicine and Clinical Research (Arts Klinisch Onderzoeker, A-KO). Studying is combined with soccer and a busy social life. As a resident in the surgical department of the Máxima Medical Center, Tijmen gets inspired by dr. R. Roumen and dr. M. Scheltinga for the Anterior Cutaneous Nerve Entrapment Syndrome.

Tijmen graduates in 2011 after studying the prevalence of ACNES within a population of patients diagnosed with the irritable bowel syndrome. This project forms the start of his $\mathrm{PhD}$-thesis about this fascinating, but underexposed syndrome. Because the limited financial resources for research in a peripheral hospital, such as the Máxima Medical Center, the research work is done in his own time and it is combined with a fulltime job at the Emergency Department. The irregular schedules appear to be the start of a new hobby on the race bike.

From the beginning of 2015 Tijmen starts a new challenge within the Sports Department. After a year in residency, Tijmen hopes to start training for Sports Medicine physician.
De auteur van dit proefschrift, Tijmen van Assen is op 3 maart 1986 geboren in Epe, een dorp gelegen in het noordoostelijke gedeelte van de Veluwe. In Epe is Tijmen opgegroeid en heeft hij de basisschool en middelbare school doorlopen.

$\mathrm{Na}$ het atheneum aan de Regionale Scholengemeenschap Noord Oost Veluwe, start het studerende leven voor hem in 2004 in Enschede, alwaar in 3 jaar de bachelor Technische Geneeskunde is afgerond.

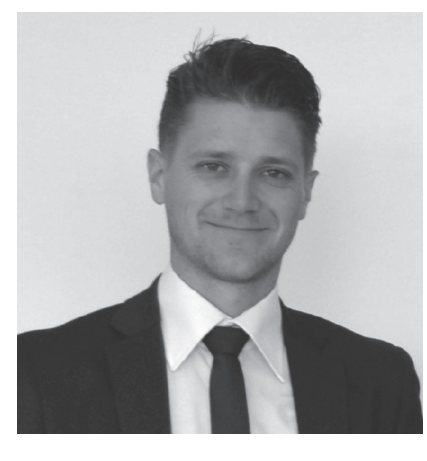

$\mathrm{Na}$ de bachelor verhuist Tijmen naar Maastricht voor de 4-jarige masteropleiding tot Arts en Klinisch Onderzoeker (A-KO). Naast een gezellig studentenleven en voetbal op zowel het veld als in de zaal, wordt ook hard gestudeerd. Tijdens het co-schap chirurgie in het Máxima Medisch Centrum in Veldhoven wordt Tijmen gegrepen door het overweldigende enthousiasme van Rudi Roumen en Marc Scheltinga, beiden chirurg, rondom het ACNES syndroom.

Met het afstudeeronderzoek naar de prevalentie van ACNES binnen de prikkelbare darmsyndroom populatie wordt in september 2011 het artsexamen behaald. Dit onderzoek vormt tevens de basis voor het proefschrift naar dit fascinerende en desondanks onderbelichte ziektebeeld. Gezien de beperkte financiële middelen voor een promovendus in een perifeer ziekenhuis, zoals het Máxima Medisch Centrum, wordt het onderzoek in eigen tijd uitgevoerd en gecombineerd met een fulltime ANIOS functie op de spoedeisende hulp / escalatiedienst. De onregelmatige diensten blijken tevens het ideale ingrediënt te zijn voor een nieuwe liefde op de racefiets.

Vanaf 1 januari 2015 slaat Tijmen een nieuwe weg in en wel binnen de sportgeneeskunde. Na een jaar als ANIOS hoopt hij zijn weg te gaan vervolgen binnen dit vakgebied om uiteindelijk tot sportarts te worden opgeleid. 